Cahiers du MONDE RUSSE

\section{Cahiers du monde russe}

Russie - Empire russe - Union soviétique et États indépendants

$55 / 3-4 \mid 2014$

Varia

\title{
СОВЕТСКИЙ АТОМНЫЙ ПРОЕКТ В СИСТЕМЕ « КОМАНДНОЙ ЭКОНОМИКИ »
}

Le projet nucléaire soviétique au sein du système d'économie planifiée

The Soviet nuclear project within the centrally planned economic system

\section{Евгений Т. Артемов}

\section{(2) OpenEdition}

1 Journals

\section{Édition électronique}

URL : http://journals.openedition.org/monderusse/8006

DOI : 10.4000/monderusse.8006

ISSN : $1777-5388$

Éditeur

Éditions de l'EHESS

\section{Édition imprimée}

Date de publication : 1 juillet 2014

Pagination : 267-294

ISBN : 978-2-7132-2441-6

ISSN : 1252-6576

\section{Référence électronique}

Евгений Т. Артемов, « Советский атомный проект в системе « командной экономики » », Cahiers du monde russe [Онлайн], 55/3-4 | 2014, Выложить онлайн 01 juillet 2018, Наводить справки в 19 avril 2019. URL : http://journals.openedition.org/monderusse/8006 ; DOI : 10.4000/monderusse.8006

Ce document a été généré automatiquement le 19 avril 2019

(c) École des hautes études en sciences sociales 


\title{
СОВЕТСКИЙ АТОМНЫЙ ПРОЕКТ В СИСТЕМЕ « КОМАНДНОЙ ЭКОНОМИКИ ॥*
}

\author{
Le projet nucléaire soviétique au sein du système d'économie planifiée \\ The Soviet nuclear project within the centrally planned economic system
}

\section{Евгений Т. Артемов}

1 В СОВРЕМЕННОЙ ИСТОРИОГРАФИИ РАСПРОСТРАНЕНО МНЕНИЕ ОБ ИЗНАЧАЛЬНОЙ НЕСОСТОЯТЕЛЬНОСТИ СОВЕТСКОЙ ЭКОНОМИКИ. ОНО БАЗИРУЕТСЯ НА ТЕОРЕТИЧЕСКИХ ОБОБЩЕНИЯХ И ПОДТВЕРЖДАЕТСЯ МНОГОЧИСЛЕННЫМИ ФАКТАМИ. ТЕМ НЕ МЕНЕЕ, СЛОЖНО ОТРИЦАТЬ, ЧТО «СОЦИАЛИСТИЧЕСКАЯ СИСТЕМА ХОЗЯЙСТВОВАНИЯ» ОКАЗАЛАСЬ СПОСОБНОЙ ОСУЩЕСТВИТЬ МАСШТАБНУЮ МОДЕРНИЗАЦИЮ ПРОИЗВОДСТВА И В СВОЕ ВРЕМЯ ВЫВЕСТИ СОВЕТСКИЙ СОЮЗ В ЧИСЛО МИРОВЫХ НАУЧНО-ТЕХНИЧЕСКИХ ЛИДЕРОВ. В ПЕРВУЮ ОЧЕРЕДЬ ЭТО ПРОЯВИЛОСЬ В НАРАЩИВАНИИ ВОЕННО-ПРОМЫШЛЕННОГО ПОТЕНЦИАЛА СТРАНЫ. СЕГОДНЯ, БЛАГОДАРЯ НОВЕЙШИМ ИССЛЕДОВАНИЯМ, СДЕЛАН ВАЖНЫЙ ШАГ В ПОНИМАНИИ ТОГО, ПОЧЕМУ В ЭТОЙ СФЕРЕ УДАЛОСЬ ДОБИТЬСЯ УСПЕХА. ОДНАКО МНОГИЕ АСПЕКТЫ РАЗВИТИЯ ОБОРОННЫХ ОТРАСЛЕЙ ИЗУЧЕНЫ ФРАГМЕНТАРНО. НЕТ ПОЛНОЙ ЯСНОСТИ КАК ОНИ « ВПИСЫВАЛИСЬ» В СОВЕТСКУЮ ЭКОНОМИЧЕСКУЮ СИСТЕМУ. ПО КРАЙНЕЙ МЕРЕ, ЭТО УТВЕРЖДЕНИЕ, НЕСМОТРЯ НА ИМЕЮЩИЕСЯ ИССЛЕДОВАНИЯ ${ }^{1}$, СПРАВЕДЛИВО ДЛЯ АТОМНОЙ ИНДУСТРИИ.

СКАЗАННОЕ ОБЪЯСНЯЕТ ВЫБОР ТЕМЫ НАСТОЯЩЕЙ СТАТЬИ: ОНА ПОСВЯЩЕНА ФОРМИРОВАНИЮ СОВЕТСКОГО ЯДЕРНО-ОРУЖЕЙНОГО КОМПЛЕКСА И ХРОНОЛОГИЧЕСКИ ОГРАНИЧЕНА 1953 Г. ИМЕННО ТОГДА, В ХОДЕ РЕАЛИЗАЦИИ АТОМНОГО ПРОЕКТА БЫЛИ ЗАЛОЖЕНЫ ЕГО ОСНОВЫ. ЭТО ПОЗВОЛИЛО ОСУЩЕСТВИТЬ РАДИКАЛЬНОЕ НАРАЩИВАНИЕ СОВЕТСКОЙ ВОЕННОЙ МОЩИ, ЧЕГО ТАК НАСТОЙЧИВО ДОБИВАЛОСЬ ВЫСШЕЕ ПОЛИТИЧЕСКОЕ РУКОВОДСТВО СТРАНЫ. ДОСТИЖЕНИЕ ЖЕЛАЕМОГО РЕЗУЛЬТАТА ОБЕСПЕЧИЛА ЦЕЛЕНАПРАВЛЕННАЯ ПОЛИТИКА И СПЕЦИАЛЬНАЯ НАСТРОЙКА ИНСТИТУТОВ «КОМАНДНОЙ ЭКОНОМИКИ». АТОМНЫЙ ПРОЕКТ БЫЛ ПОСТАВЛЕН В ИСКЛЮЧИТЕЛЬНОЕ 
ПОЛОЖЕНИЕ. ЕМУ ПРЕДОСТАВИЛИ БЕЗУСЛОВНЫЙ ПРИОРИТЕТ В РЕСУРСНОМ ОБЕСПЕЧЕНИИ. ОТВЕТСТВЕННОСТЬ ЗА ДОСТИЖЕНИЕ КОНЕЧНОГО РЕЗУЛЬТАТА ВОЗЛОЖИЛИ НА РЕАЛЬНО НИКОМУ НЕ ПОДОТЧЕТНУЮ « УПРАВЛЕНЧЕСКУЮ ВЕРТИКАЛЬ». ОНА ПОЛУЧИЛА ПРАВО ПРИВЛЕКАТЬ К ВЫПОЛНЕНИЮ СВОИХ ЗАДАНИЙ ЛЮБЫХ ИСПОЛНИТЕЛЕЙ. ЧТОБЫ РАСШИРИТЬ ПОЛЕ ДЛЯ МАНЕВРА ПРИ РАСПРЕДЕЛЕНИИ РЕСУРСОВ И КОРРЕКТИРОВКЕ АЛГОРИТМА ПРАКТИЧЕСКИХ ДЕЙСТВИЙ ФАКТИЧЕСКИ ОТКАЗАЛИСЬ ОТ « ПЛАНОВОСТИ» В ОРГАНИЗАЦИИ ПРОИЗВОДСТВА. ВПРОЧЕМ, ОНА ВСЕГДА БЫЛА ВЕСЬМА УСЛОВНОЙ, НА ЧТО УЖЕ ДАВНО ОБРАТИЛИ ВНИМАНИЕ ИССЛЕДОВАТЕЛИ 2 . ТЕМ НЕ МЕНЕЕ, ПЛАНИРОВАНИЕ ШИРОКО ИСПОЛЬЗОВАЛОСЬ В КАЧЕСТВЕ ВНЕШНЕГО МЕХАНИЗМА КОНТРОЛЯ ЗА ДЕЯТЕЛЬНОСТЬЮ ОТРАСЛЕВЫХ ВЕДОМСТВ И ХОЗЯЙСТВУЮЩИХ СУБЪЕКТОВ. В АТОМНОМ ЖЕ ПРОЕКТЕ ОНО, ПО СУТИ, УТРАТИЛО ЭТУ ФУНКЦИЮ, ПРЕВРАТИВШИСЬ ВО ВНУТРЕННИЕ «ДЕКЛАРАЦИИ О НАМЕРЕНИЯХ». ИНАЧЕ ГОВОРЯ, РУКОВОДСТВО ИМ ОСУЩЕСТВЛЯЛОСЬ НЕ « В ПЛАНОВОМ ПОРЯДКЕ», А В « РУЧНОМ РЕЖИМЕ». ЕДИНСТВЕННОЕ, ЧТО РЕАЛЬНО КОНТРОЛИРОВАЛОСЬ ИЗВНЕ, - ЭТО СРОКИ СОЗДАНИЯ ОПЫТНОГО ЯДЕРНОГО ЗАРЯДА, А ПОЗДНЕЕ - МАСШТАБЫ СЕРИЙНОГО ПРОИЗВОДСТВА « ИЗДЕЛИЙ». ПОДОБНЫЕ ПОДХОДЫ, В СИЛУ СВОЕЙ ЭКСТРАОРДИНАРНОСТИ, МОГЛИ ТИРАЖИРОВАТЬСЯ ЛИШЬ В ОГРАНИЧЕННЫХ МАСШТАБАХ. ДАЖЕ ПРИ СОЗДАНИИ РАКЕТНОЙ И КОСМИЧЕСКОЙ ТЕХНИКИ, СИСТЕМЫ ПРОТИВОВОЗДУШНОЙ ОБОРОНЫ МОСКВЫ, РАДИОЭЛЕКТРОННОГО ВООРУЖЕНИЯ И РЕШЕНИИ АНАЛОГИЧНЫХ ПРИОРИТЕТНЫХ ЗАДАЧ, УКАЗАННЫЕ ПОДХОДЫ ИСПОЛЬЗОВАЛИСЬ ЧАСТИЧНО. ДРУГИМИ СЛОВАМИ, ОПЫТ АТОМНОГО ПРОЕКТА В КОНСТРУИРОВАНИИ СПЕЦИАЛЬНЫХ УПРАВЛЕНЧЕСКИХ МЕХАНИЗМОВ, В ОРГАНИЗАЦИИ РАБОТЫ И МОТИВАЦИИ ТРУДА ОКАЗАЛСЯ НЕВОСПРОИЗВОДИМЫМ В ПОЛНОМ ОБЪЕМЕ.

ИСТОЧНИКОВАЯ БАЗА ИСТОРИИ СОВЕТСКОГО АТОМНОГО ПРОЕКТА ВЕСЬМА СПЕЦИФИЧНА. ОСНОВНЫЕ АРХИВНЫЕ ДОКУМЕНТАЛЬНЫЕ КОМПЛЕКСЫ НЕДОСТУПНЫ ИССЛЕДОВАТЕЛЯМ. ОДНАКО В ПОСЛЕДНИЕ ГОДЫ ОПУБЛИКОВАН БОЛЬШОЙ МАССИВ РАНЕЕ СЕКРЕТНЫХ МАТЕРИАЛОВ. ПРЕЖДЕ ВСЕГО РЕЧЬ ИДЕТ ОБ « ОФИЦИАЛЬНОМ» СБОРНИКЕ ДОКУМЕНТОВ (3 ТОМА В 12 КНИГАХ) «ПО ИСТОРИИ СОЗДАНИЯ ЯДЕРНОГО ОРУЖИЯ В СССР» ${ }^{3}$ В НЕГО ВКЛЮЧЕНЫ ПОСТАНОВЛЕНИЯ И РАСПОРЯЖЕНИЯ ПРАВИТЕЛЬСТВА СТРАНЫ, ПРОТОКОЛЫ ЗАСЕДАНИЙ СПЕЦИАЛЬНОГО КОМИТЕТА ПРИ СОВЕТЕ МИНИСТРОВ СССР И ЕГО ТЕХНИЧЕСКОГО И ИНЖЕНЕРНО-ТЕХНИЧЕСКОГО СОВЕТОВ, ДЕЛОВАЯ ПЕРЕПИСКА, ИНФОРМАЦИОННЫЕ И ОТЧЕТНЫЕ МАТЕРИАЛЫ ПЕРВОГО ГЛАВНОГО УПРАВЛЕНИЯ ИСПОЛНИТЕЛЬНОГО ОРГАНА АТОМНОГО ПРОЕКТА И Т.Д. СТОИТ ТАКЖЕ ОТМЕТИТЬ ИЗДАНИЕ (8 ТОМОВ В 11 КНИГАХ) РАССЕКРЕЧЕННЫХ АРХИВНЫХ ДОКУМЕНТОВ ИЗ ФОНДОВ РОССИЙСКОГО ФЕДЕРАЛЬНОГО ЯДЕРНОГО ЦЕНТРА - ВНИИ ЭКСПЕРИМЕНТАЛЬНОЙ ФИЗИКИ (Г. САРОВ $)^{4}$. ЭТИ ПУБЛИКАЦИИ, НАРЯДУ С ОБШИРНОЙ МЕМУАРНОЙ ЛИТЕРАТУРОЙ, ПОЗВОЛЯЮТ ДОСТАТОЧНО ПОЛНО ОСВЕТИТЬ ПРИНЦИПИАЛЬНЫЕ ОСОБЕННОСТИ ОРГАНИЗАЦИИ РАБОТЫ, РЕАЛИЗОВАННЫЕ В СОВЕТСКОМ АТОМНОМ ПРОЕКТЕ.

\section{У истоков атомного проекта}

4 «ЗАПУСК» СОВЕТСКОГО АТОМНОГО ПРОЕКТА ЧАСТО ОТНОСЯТ К АВГУСТУ 1945 Г. В ДЕЙСТВИТЕЛЬНОСТИ ЖЕ, РАБОТЫ ПО ЕГО РЕАЛИЗАЦИИ БЫЛИ НАЧАТЫ ТРЕМЯ ГОДАМИ РАНЬШЕ. ЭТО ПРОИЗОШЛО ПРИ СЛЕДУЮЩИХ ОБСТОЯТЕЛЬСТВАХ. НАЧИНАЯ С 1920-Х ГГ. СОВЕТСКИЕ УЧЕНЫЕ АКТИВНО ВЕЛИ ЯДЕРНО-ФИЗИЧЕСКИЕ ИССЛЕДОВАНИЯ И ДОБИЛИСЬ ПРИЗНАННЫХ РЕЗУЛЬТАТОВ 5 . ИХ, КАК И ВСЕ МИРОВОЕ НАУЧНОЕ СООБЩЕСТВО, БУКВАЛЬНО ПОТРЯСЛО ОТКРЫТИЕ, СДЕЛАННОЕ В КОНЦЕ 1938 Г.: ТОГДА БЫЛО 
УСТАНОВЛЕНО, ЧТО ПРИ ДЕЛЕНИИ ЯДРА УРАНА ЧАСТЬ ЕГО МАССЫ ПРЕВРАЩАЕТСЯ В ЭНЕРГИЮ, И, СЛЕДОВАТЕЛЬНО, ИМЕЕТСЯ ПРИНЦИПИАЛЬНАЯ ВОЗМОЖНОСТЬ ЕЕ ИСПОЛЬЗОВАНИЯ В ПРАКТИЧЕСКИХ ЦЕЛЯХ, В ТОМ ЧИСЛЕ В ВОЕННОМ ДЕЛЕ. НО ЗДЕСЬ ВОЗНИКАЛИ ЧРЕЗВЫЧАЙНО СЛОЖНЫЕ ПРОБЛЕМЫ ФУНДАМЕНТАЛЬНОГО И ТЕХНИЧЕСКОГО СВОЙСТВА. ПРИЗНАННЫЕ НАУЧНЫЕ АВТОРИТЕТЫ ОТНОСИЛИ ИХ РЕШЕНИЕ В НЕОПРЕДЕЛЕННОЕ БУДУЩЕЕ. КАК ЗАЯВЛЯЛ АКАДЕМИК П.Л. КАПИЦА, « МОЖНО СКАЗАТЬ, ЧТО АТОМНОЙ ЭНЕРГИЕЙ... МЫ НЕ ВОСПОЛЬЗУЕМСЯ С БОЛЬШОЙ ЛЕГКОСТЬЮ, А ПО ВСЕЙ ВИДИМОСТИ, И НЕ ВОСПОЛЬЗУЕМСЯ СОВСЕМ» ${ }^{6}$.

КРИТИЧЕСКОЕ ОТНОШЕНИЕ ВЕДУЩИХ СОВЕТСКИХ ФИЗИКОВ К ПРИКЛАДНЫМ АСПЕКТАМ ЯДЕРНО-ФИЗИЧЕСКИХ ИССЛЕДОВАНИЙ ИМЕЛО ВАЖНЫЕ ПОСЛЕДСТВИЯ : С НАЧАЛОМ ВЕЛИКОЙ ОТЕЧЕСТВЕННОЙ ВОЙНЫ ПО РЕШЕНИЮ ПРЕЗИДИУМА АН СССР ИХ СВЕРНУЛИ КАК НЕ ИМЕЮЩИЕ «АКТУАЛЬНОГО ЗНАЧЕНИЯ ДЛЯ ОБОРОНЫ СТРАНЫ». ПРАВДА В АКАДЕМИЧЕСКОЙ СРЕДЕ ВЫСКАЗЫВАЛИСЬ МНЕНИЯ О НЕРАЗУМНОСТИ ТАКОГО ШАГА. В СОВРЕМЕННОЙ ИСТОРИОГРАФИИ УТВЕРЖДАЕТСЯ, ЧТО Г.Н. ФЛЕРОВ, СОТРУДНИК ЛЕНИНГРАДСКОГО ФИЗИКО-ТЕХНИЧЕСКОГО ИНСТИТУТА АН СССР, В НАЧАЛЕ 1942 Г. ОТПРАВИЛ РЯД ПИСЕМ «В САМЫЕ ВЫСОКИЕ ИНСТАНЦИИ», В КОТОРЫХ ПРЕДЛАГАЛ « НЕМЕДЛЕННО РАЗВЕРНУТЬ» РАБОТЫ ПО АТОМНОЙ ПРОБЛЕМЕ. ЕГО ИНФОРМАЦИЯ БУДТО БЫ «СРАБОТАЛА», И «ДЕЛО СДВИНУЛОСЬ С МЕРТВОЙ ТОЧКИ». ОДНАКО ДОКУМЕНТАЛЬНЫХ СВИДЕТЕЛЬСТВ, ПОДТВЕРЖДАЮЩИХ ЭТО УТВЕРЖДЕНИЕ, НЕ ПРИВОДИТСЯ. ДОСТОВЕРНО ИЗВЕСТНО ОДНО: ИНИЦИАТИВУ Г.Н. ФЛЕРОВА НЕ ПОДДЕРЖАЛИ АКАДЕМИКИ А.Ф. ИОФФЕ И П.Л. КАПИЦА. И НИКАКИХ ПРАКТИЧЕСКИХ ДЕЙСТВИЙ ЗА НЕЙ НЕ ПОСЛЕДОВАЛО.

В ТО ЖЕ ВРЕМЯ В СТРАНАХ - СОЮЗНИКАХ СССР ПО АНТИГИТЛЕРОВСКОЙ КОАЛИЦИИ СКЛАДЫВАЛАСЬ ИНАЯ СИТУАЦИЯ : РАБОТЫ ПО ОВЛАДЕНИЮ ЯДЕРНОЙ ЭНЕРГИЕЙ ПРОВОДИЛИСЬ С НАРАСТАЮЩИМ РАЗМАХОМ. ИХ РЕЗУЛЬТАТОМ СТАЛ ВЫВОД О ВОЗМОЖНОСТИ СОЗДАНИЯ АТОМНОЙ БОМБЫ В КРАТЧАЙШИЕ СРОКИ. ЭТУ ИНФОРМАЦИЮ УДАЛОСЬ ПОЛУЧИТЬ СОВЕТСКОЙ РАЗВЕДКЕ. ОСЕНЬЮ 1941 Г. НА ЕЕ ОСНОВАНИИ НКВД ПОДГОТОВИЛ ДОКЛАДНУЮ ЗАПИСКУ НА ИМЯ И.В. СТАЛИНА. В НЕЙ ПОДНИМАЛСЯ ВОПРОС О РАЗВЕРТЫВАНИИ РАБОТ ПО ОВЛАДЕНИЮ ЯДЕРНОЙ ЭНЕРГИЕЙ В СОВЕТСКОМ СОЮЗЕ. НО ТОГДА ЗАПИСКУ, КАК И ЕЕ СЛЕДУЮЩИЙ ВАРИАНТ, ПОЯВИВШИЙСЯ В МАРТЕ 1942 Г., НЕ ОТПРАВИЛИ АДРЕСАТУ 9 . ВИДИМО, Л.П. БЕРИЯ СЧЕЛ НЕСВОЕВРЕМЕННЫМ СТАВИТЬ ПЕРЕД СТАЛИНЫМ ПЕРСПЕКТИВНЫЕ ВОПРОСЫ, ОСНОВЫВАЯСЬ НА НЕ ВПОЛНЕ ПРОВЕРЕННЫХ СВЕДЕНИЯХ. ТЕМ НЕ МЕНЕЕ, ПОСТУПИВШУЮ ИНФОРМАЦИЮ ПРИЗНАЛИ ВЕСЬМА ВАЖНОЙ. ЗАРУБЕЖНАЯ АГЕНТУРА ПОЛУЧИЛА ПРЕДПИСАНИЕ «ЗАНЯТЬСЯ» АТОМНОЙ ПРОБЛЕМОЙ « СО ВСЕЙ СЕРЬЕЗНОСТЬЮ» И ПРОВЕСТИ « РАЗРАБОТКУ СОПРИКАСАЮЩИХСЯ» С НЕЙ « ЛИЦ В ЦЕЛЯХ ПОЛУЧЕНИЯ ТЕХНОЛОГИЧЕСКИХ РАСЧЕТНЫХ ДАННЫХ ПО САМОМУ ПРОЦЕССУ, АППАРАТУРЕ И МЕХАНИЗМАМ, СХЕМ И ЧЕРТЕЖЕЙ, ЭКОНОМИЧЕСКИХ ОБОСНОВАНИЙ ПРОИЗВОДИМЫХ РАБОТ ${ }^{10}$.

7 ПРИНЯТЫЕ МЕРЫ ДАЛИ СВОИ РЕЗУЛЬТАТЫ. ПО «ЗАКРЫТЫМ КАНАЛАМ» ИЗ ВЕЛИКОБРИТАНИИ И США СТАЛИ ПОСТУПАТЬ В РАСТУЩИХ МАСШТАБАХ СВЕДЕНИЯ О ПРОВЕДЕНИИ РАБОТ В ОБЛАСТИ ОВЛАДЕНИЯ АТОМНОЙ ЭНЕРГИЕЙ. СПЕЦСЛУЖБЫ ПЫТАЛИСЬ ИХ ПЕРЕПРОВЕРИТЬ, ИСПОЛЬЗУЯ В КАЧЕСТВЕ ЭКСПЕРТОВ ВЕДУЩИХ УЧЕНЫХ. НО ТЕ ЗАНЯЛИ «ОСТОРОЖНУЮ» ПОЗИЦИЮ. ТАК, АКАДЕМИК В.Г. ХЛОПИН В ОТВЕТ НА ЗАПРОС ГЛАВНОГО РАЗВЕДЫВАТЕЛЬНОГО УПРАВЛЕНИЯ ГЕНШТАБА КРАСНОЙ АРМИИ В МАЕ 1942 Г. ПИСАЛ : «ПО НАШЕМУ МНЕНИЮ, ВОЗМОЖНОСТЬ ИСПОЛЬЗОВАНИЯ 
ВНУТРИАТОМНОЙ ЭНЕРГИИ ДЛЯ ВОЕННЫХ ЦЕЛЕЙ (В ТЕЧЕНИЕ НАСТОЯЩЕЙ ВОЙНЫ) ВЕСЬМА МАЛОВЕРОЯТНА ${ }^{11}$. ОТСЮДА МОЖНО БЫЛО СДЕЛАТЬ ЛИШЬ ОДИН ВЫВОД: ВОЗОБНОВЛЕНИЕ ЯДЕРНО-ФИЗИЧЕСКИХ ИССЛЕДОВАНИЙ - ДЕЛО ОТДАЛЕННОГО БУДУЩЕГО. ИНАЧЕ ВОСПРИНЯЛИ ПОСТУПИВШУЮ ИЗ-ЗА РУБЕЖА ИНФОРМАЦИЮ В ВЫСШИХ ЭШЕЛОНАХ ВЛАСТИ. ПО СВИДЕТЕЛЬСТВУ М.Г. ПЕРВУХИНА, ЗАНИМАВШЕГО ТОГДА ПОСТ ЗАМЕСТИТЕЛЯ ПРЕДСЕДАТЕЛЯ СНК СССР, ВЕСНОЙ 1942 Г. ОНА ДОШЛА ДО В.М. МОЛОТОВА ${ }^{12}$. ТОТ ТАКЖЕ РЕШИЛ ПРОКОНСУЛЬТИРОВАТЬСЯ С УЧЕНЫМИ, ПРИЗНАННЫМИ НАУЧНЫМИ АВТОРИТЕТАМИ, - АКАДЕМИКАМИ П.Л. КАПИЦЕЙ И А.Ф. ИОФФЕ. СПУСТЯ ТРИДЦАТЬ ЛЕТ В.М. МОЛОТОВ ТАК РАССКАЗЫВАЛ О СОСТОЯВШИХСЯ ТОГДА РАЗГОВОРАХ: «ВЫЗВАЛ КАПИЦУ К СЕБЕ, АКАДЕМИКА. ОН СКАЗАЛ, ЧТО МЫ К ЭТОМУ НЕ ГОТОВЫ, И АТОМНАЯ БОМБА - ОН ТАКЖЕ КАК-ТО НЕЯСНО К ЭТОМУ ОТНЕССЯ» ${ }^{13}$. ПРАВДА, С САМИМИ РАЗВЕДДАННЫМИ УЧЕНЫХ НЕ ЗНАКОМИЛИ. СОГЛАСНО СОХРАНИВШЕМУСЯ СВИДЕТЕЛЬСТВУ РУКОВОДИТЕЛЯ НАУЧНО-ТЕХНИЧЕСКОЙ РАЗВЕДКИ НКВД Л.Р. КВАСНИКОВА, ИМ ПОКАЗАЛИ ТОЛЬКО РЕФЕРАТ АГЕНТУРНЫХ МАТЕРИАЛОВ. УВИДЕННОЕ НЕ ПРОИЗВЕЛО ДОЛЖНОГО ВПЕЧАТЛЕНИЯ НИ НА П.Л.КАПИЦУ, НИ НА А.Ф. ИОФФЕ. ОТСЮДА ИХ СДЕРЖАННЫЕ ОЦЕНКИ ПЕРСПЕКТИВ СОЗДАНИЯ АТОМНОЙ БОМБЫ 14 .

ТЕМ НЕ МЕНЕЕ, В.М. МОЛОТОВ ВЗЯЛ НА СЕБЯ ОТВЕТСТВЕННОСТЬ ЗА « ЗАПУСК» РАБОТ ПО ОВЛАДЕНИЮ АТОМНОЙ ЭНЕРГИЕЙ. В ЖЕСТКО ЦЕНТРАЛИЗОВАННОЙ СИСТЕМЕ ПОДДЕРЖКА ОДНОГО ИЗ ВЫСШИХ ГОСУДАРСТВЕННЫХ РУКОВОДИТЕЛЕЙ ЗНАЧИЛА ОЧЕНЬ МНОГО : ОНА ПОЗВОЛЯЛА ИГНОРИРОВАТЬ ЛЮБЫЕ, ДАЖЕ ОТРИЦАТЕЛЬНЫЕ ЗАКЛЮЧЕНИЯ САМЫХ АВТОРИТЕТНЫХ ЭКСПЕРТОВ. НО В ДАННОМ СЛУЧАЕ ЭТО ПРИНЕСЛО ПОЛОЖИТЕЛЬНЫЕ РЕЗУЛЬТАТЫ. ПОДГОТОВЛЕННЫЙ ПО УКАЗАНИЮ В.М. МОЛОТОВА ПРОЕКТ РАСПОРЯЖЕНИЯ ГКО « ОБ ОРГАНИЗАЦИИ РАБОТ ПО УРАНУ» 27 СЕНТЯБРЯ 1942 Г. НАПРАВИЛИ И.В. СТАЛИНУ, КОТОРЫЙ ПОДПИСАЛ ЕГО НА СЛЕДУЮЩИЙ ДЕНЬ ${ }^{15}$. ТАК БЫЛ « ЗАПУЩЕН» СОВЕТСКИЙ АТОМНЫЙ ПРОЕКТ.

СНАЧАЛА РЕШЕНИЕ АТОМНОЙ ПРОБЛЕМЫ ВОЗГЛАВИЛ ВИЦЕ-ПРЕЗИДЕНТ АКАДЕМИИ НАУК А.Ф.ИОФФЕ. НО УЖЕ В ФЕВРАЛЕ 1943 Г. В СВЯЗИ С «НЕУДОВЛЕТВОРИТЕЛЬНЫМ СОСТОЯНИЕМ» ЕГО ОТСТРАНИЛИ ОТ ДЕЛ, « ПОВСЕДНЕВНОЕ РУКОВОДСТВО» АТОМНЫМ ПРОЕКТОМ ВОЗЛОЖИЛИ НА М.Г. ПЕРВУХИНА, А НАУЧНОЕ - НА И.В. КУРЧАТОВА ${ }^{16}$. ЭТО БЫЛ НЕСТАНДАРТНЫЙ ХОД. ВО-ПЕРВЫХ, СОГЛАСНО УСТАНОВЛЕННОМУ ПОРЯДКУ», ВЫПОЛНЕНИЕ ТАКОГО ПОРУЧЕНИЯ СЛЕДОВАЛО ПЕРЕДАТЬ КОНКРЕТНОМУ ВЕДОМСТВУ. В ДКАДЕМИИ НАУК СССР, ЯВЛЯВШЕЙСЯ В СОВЕТСКОЙ СИСТЕМЕ СВОЕОБРАЗНЫМ МИНИСТЕРСТВОМ НАУКИ. ВНАЧАЛЕ ТАК И СДЕЛАЛИ. АКАДЕМИИ НАУК ПРЕДЛОЖИЛИ «ВОЗОБНОВИТЬ ИССЛЕДОВАНИЯ В ОБЛАСТИ ИСПОЛЬЗОВАНИЯ АТОМНОЙ ЭНЕРГИИ» И « ПРЕДСТАВИТЬ В ГОСУДАРСТВЕННЫЙ КОМИТЕТ ОБОРОНЫ ДОКЛАД О ВОЗМОЖНОСТИ СОЗДАНИЯ УРАНОВОЙ БОМБЫ ИЛИ УРАНОВОГО ТОПЛИВА» ${ }^{17}$. НО ЕЕ РУКОВОДСТВО НЕ ВИДЕЛО ЗДЕСЬ ПЕРСПЕКТИВ, ЧТО ПРОВОЦИРОВАЛО РАЗЛИЧНЫЕ ОРГАНИЗАЦИОННЫЕ НЕУВЯЗКИ И ЗАТЯГИВАНИЕ СООТВЕТСТВУЮЩЕЙ РАБОТЫ. ПОЭТОМУ РУКОВОДСТВО РАБОТОЙ ПО ОВЛАДЕНИЮ ВНУТРИАТОМНОЙ ЭНЕРГИЕЙ, ВОПРЕКИ ОБЫЧНОЙ ПРАКТИКЕ, ВОЗЛОЖИЛИ НЕПОСРЕДСТВЕННО НА ПРАВИТЕЛЬСТВО. ВО-ВТОРЫХ, НАУЧНЫМ РУКОВОДИТЕЛЕМ ПРОЕКТА ГОСУДАРСТВЕННОЙ ВАЖНОСТИ НАЗНАЧИЛИ РЯДОВОГО ЗАВЕДУЮЩЕГО ЛАБОРАТОРИЕЙ, ДОКТОРА НАУК. ТАКОЙ ФОРМАЛЬНЫЙ СТАТУС, КОНЕЧНО, СОЗДАВАЛ И.В. КУРЧАТОВУ ОПРЕДЕЛЕННЫЕ ТРУДНОСТИ. НЕ СЛУЧАЙНО ПРИ ПРЯМОЙ ПОДДЕРЖКЕ ПРАВИТЕЛЬСТВА В СЕНТЯБРЕ 1943 Г., МИНУЯ ЗВАНИЕ ЧЛЕНА-КОРРЕСПОНДЕНТА АН СССР, ОН БЫЛ СРАЗУ ИЗБРАН АКАДЕМИКОМ ${ }^{18}$. 
ОДНАКО ПРИНЯТЫЕ МЕРЫ НЕ УСТРАНИЛИ ВСЕХ ОРГАНИЗАЦИОННЫХ ПРОБЛЕМ. РАСТУЩИЕ МАСШТАБЫ РЕАЛИЗАЦИИ АТОМНОГО ПРОЕКТА ТРЕБОВАЛИ ПРИВЛЕЧЕНИЯ МНОГОЧИСЛЕННЫХ ИСПОЛНИТЕЛЕЙ РАЗНОЙ ВЕДОМСТВЕННОЙ ПРИНАДЛЕЖНОСТИ, МОБИЛИЗАЦИИ ЗНАЧИТЕЛЬНЫХ МАТЕРИАЛЬНЫХ РЕСУРСОВ. ДЛЯ М.Г. ПЕРВУХИНА И И.В. КУРЧАТОВА - В СИЛУ ИХ НЕДОСТАТОЧНОГО ПОЛИТИЧЕСКОГО И АППАРАТНОГО ВЕСА - ЗАДАЧА ОКАЗАЛАСЬ НЕПОСИЛЬНОЙ. ВЫХОД НАШЛИ В «УКРЕПЛЕНИИ» РУКОВОДСТВА АТОМНОГО ПРОЕКТА: В ДЕКАБРЕ 1944 Г. «НАБЛЮДЕНИЕ» ЗА НИМ ВОЗЛОЖИЛИ ПЕРСОНАЛЬНО НА Л.П. БЕРИЮ КАК ПРЕДСЕДАТЕЛЯ ОПЕРАТИВНОГО БЮРО ГКО, КООРДИНИРОВАВШЕГО ВОЕННО-ПРОМЫШЛЕННУЮ ЧАСТЬ ЭКОНОМИКИ СТРАНЫ ${ }^{19}$.

11 В СЕРЕДИНЕ МАЯ 1945 Г. Л.П. БЕРИЯ И И.В. КУРЧАТОВ ДОЛОЖИЛИ И.В.СТАЛИНУ О ГОТОВНОСТИ ПЕРЕЙТИ К ПРАКТИЧЕСКОМУ РЕШЕНИЮ АТОМНОЙ ПРОБЛЕМЫ. ОНИ ПРЕДЛОЖИЛИ АЛГОРИТМ СООТВЕТСТВУЮЩИХ ДЕЙСТВИЙ- ОТ СТРОИТЕЛЬСТВА ПРЕДПРИЯТИЙ ПО ДОБЫЧЕ УРАНОВОЙ РУДЫ И ЕЕ ПЕРЕРАБОТКИ ДО ПРОЕКТИРОВАНИЯ ПРОИЗВОДСТВ ПО ПОЛУЧЕНИЮ «АТОМНОЙ ВЗРЫВЧАТКИ» (УРАНА-235, ПЛУТОНИЯ) И ВЫДАЧИ ТЕХНИЧЕСКОГО ЗАДАНИЯ НА ИЗГОТОВЛЕНИЕ ЯДЕРНОЙ БОМБЫ. ПРЕДСТАВЛЕННЫЕ ИМИ ПЛАНЫ СРАЗУ ЖЕ БЫЛИ УТВЕРЖДЕНЫ СПЕЦИАЛЬНЫМИ ПОСТАНОВЛЕНИЯМИ ГКО ${ }^{20}$. НО ВСКОРЕ К НИМ СНОВА ПРИШЛОСЬ ВЕРНУТЬСЯ. ПОСЛЕ АТОМНОЙ БОМБАРДИРОВКИ ХИРОСИМЫ И НАГАСАКИ ВЫСШЕЕ РУКОВОДСТВО СТРАНЫ СДЕЛАЛО ВЫВОД О НЕОБХОДИМОСТИ ФОРСИРОВАТЬ СОЗДАНИЕ СОБСТВЕННОГО ЯДЕРНОГО ОРУЖИЯ. ПУТИ РЕШЕНИЯ ЭТОЙ ЗАДАЧИ БЫЛИ ОПРЕДЕЛЕНЫ ПОСТАНОВЛЕНИЕМ ГОСУДАРСТВЕННОГО КОМИТЕТА ОБОРОНЫ « О СПЕЦИАЛЬНОМ КОМИТЕТЕ ПРИ ГОКО» ОТ 20 АВГУСТА $1945 \Gamma^{21}$

\section{Выбор стратегии}

ПО СВОЕМУ СОДЕРЖАНИЮ ПРИНЯТОЕ ПОСТАНОВЛЕНИЕ ЯВЛЯЛОСЬ ПОЛИТИЧЕСКИМ ДОКУМЕНТОМ : В НЕМ РАБОТЫ « ПО ИСПОЛЬЗОВАНИЮ ВНУТРИАТОМНОЙ ЭНЕРГИИ УРАНА» ОБЪЯВИЛИ ВЫСШИМ ГОСУДАРСТВЕННЫМ ПРИОРИТЕТОМ. ДАЖЕ В ОФИЦИАЛЬНЫХ ДОКУМЕНТАХ СОЗДАНИЕ ЯДЕРНОГО ОРУЖИЯ СТАЛО НАЗЫВАТЬСЯ «ЗАДАЧЕЙ НОМЕР ОДИН». КОМАНДНАЯ ЭКОНОМИКА ПОЗВОЛЯЛА ИЗЫСКАТЬ ДЛЯ ЕЕ РЕШЕНИЯ НЕОБХОДИМЫЕ РЕСУРСЫ, В ТОМ ЧИСЛЕ ЗА СЧЕТ УЩЕМЛЕНИЯ ИНТЕРЕСОВ ЛЮБЫХ ОТРАСЛЕЙ ЭКОНОМИКИ, ПОТРЕБИТЕЛЬСКОГО СЕКТОРА. НО ДЛЯ ЭТОГО ТРЕБОВАЛОСЬ ВЫПОЛНИТЬ РЯД УСЛОВИЙ; ВО-ПЕРВЫХ, СОЗДАТЬ ЖЕСТКУЮ УПРАВЛЕНЧЕСКУЮ ВЕРТИКАЛЬ ДЛЯ ДОСТИЖЕНИЯ ЗАЯВЛЕННЫХ ЦЕЛЕЙ, ВО-ВТОРЫХ, НАДЕЛИТЬ ЕЕ ШИРОКИМИ ПОЛНОМОЧИЯМИ В ПЛАНЕ ПЕРЕРАСПРЕДЕЛЕНИЯ РЕСУРСОВ В ОБЩЕГОСУДАРСТВЕННОМ МАСШТАБЕ И, В-ТРЕТЬИХ, ПОСЛЕДОВАТЕЛЬНО И ТВЕРДО ИСПОЛЬЗОВАТЬ АДМИНИСТРАТИВНЫЕ САНКЦИИ ЗА НЕНАДЛЕЖАЩЕЕ ИСПОЛНЕНИЕ СПУСКАЕМЫХ “СВЕРХУ» ЗАДАНИЙ. ОДНАКО ОДНОЙ МОБИЛИЗАЦИИ СОБСТВЕННЫХ СИЛ БЫЛО ЕЩЕ НЕДОСТАТОЧНО, ЧТОБЫ ДОБИТЬСЯ РЕЗУЛЬТАТА В ПРИЕМЛЕМЫЕ ДЛЯ РУКОВОДСТВА СТРАНЫ СРОКИ. ДЛЯ УСКОРЕНИЯ РАБОТ И ПОЛУЧЕНИЯ ГАРАНТИРОВАННОГО РЕЗУЛЬТАТА СДЕЛАЛИ СТАВКУ НА МАКСИМАЛЬНОЕ ИСПОЛЬЗОВАНИЕ ЗАРУБЕЖНЫХ НАУЧНО-ТЕХНИЧЕСКИХ ДОСТИЖЕНИЙ. ИНОЙ ВАРИАНТ ДЕЙСТВИЙ, ПО СВИДЕТЕЛЬСТВУ ГЛАВНОГО КОНСТРУКТОРА ПЕРВОЙ СОВЕТСКОЙ АТОМНОЙ БОМБЫ Ю.Б. ХАРИТОНА, ОЗНАЧАЛ УВЕЛИЧЕНИЕ ВЕРОЯТНОСТИ НЕУДАЧИ, ЧТО ЯВИЛОСЬ БЫ « НЕПОЗВОЛИТЕЛЬНЫМ И ЛЕГКОМЫСЛЕННЫМ ШАГОМ»²2. 
ЗАИМСТВОВАНИЕ ИНОСТРАННЫХ ТЕХНОЛОГИЙ ОСУЩЕСТВЛЯЛОСЬ ПО РАЗЛИЧНЫМ КАНАЛАМ. МНОГО ДАЛА ТЕХНИЧЕСКАЯ ДОКУМЕНТАЦИЯ, ПОЛУЧЕННАЯ В СЧЕТ РЕПАРАЦИЙ. ИЗ СОВЕТСКОЙ ЗОНЫ ОККУПАЦИИ ГЕРМАНИИ ВЫВЕЗЛИ ЗНАЧИТЕЛЬНУЮ ЧАСТЬ ОБОРУДОВАНИЯ И СПЕЦИАЛЬНЫХ МАТЕРИАЛОВ, НЕ ПРОИЗВОДИВШИХСЯ ТОГДА В СССР. ВЕСЬМА ПРИГОДИЛСЯ В АТОМНОМ ПРОЕКТЕ ОПЫТ НЕМЕЦКИХ СПЕЦИАЛИСТОВ (ПРИГЛАШЕННЫХ И ПЛЕННЫХ). ОТДЕЛЬНЫЕ ВИДЫ УНИКАЛЬНОГО ПРИБОРНОГО ОБОРУДОВАНИЯ УДАЛОСЬ ПОЛУЧИТЬ ПО ЛЕНД-ЛИЗУ ${ }^{23}$. НО ГЛАВНУЮ РОЛЬ В ОПРЕДЕЛЕНИИ ПУТЕЙ СОЗДАНИЯ АТОМНОЙ БОМБЫ СЫГРАЛИ РАЗВЕДДАННЫЕ. ОНИ СОДЕРЖАЛИ СВЕДЕНИЯ О ПРИНЦИПИАЛЬНОМ УСТРОЙСТВЕ АТОМНОЙ БОМБЫ, ЯДЕРНО-ФИЗИЧЕСКИХ СВОЙСТВАХ ДЕЛЯЩИХСЯ МАТЕРИАЛОВ, ТЕХНОЛОГИЯХ РАЗДЕЛЕНИЯ ИЗОТОПОВ УРАНА, КОНСТРУКЦИЯХ И ПАРАМЕТРАХ ЯДЕРНЫХ РЕАКТОРОВ, ОРГАНИЗАЦИИ РАБОТЫ ПО СОЗДАНИЮ АТОМНОГО ОРУЖИЯ. ЭТА ИНФОРМАЦИЯ ПОЗВОЛИЛА СОВЕТСКОЙ СТОРОНЕ СВОЕВРЕМЕННО КОРРЕКТИРОВАТЬ СОБСТВЕННЫЕ НАПРАВЛЕНИЯ НАУЧНОГО ПОИСКА, СОКРАТИТЬ СРОКИ РАЗРАБОТКИ ТЕХНОЛОГИЙ И СОЗДАНИЯ СООТВЕТСТВУЮЩИХ ПРОИЗВОДСТВ 24 . ДРУГИМИ СЛОВАМИ, ПЕРВАЯ СОВЕТСКАЯ АТОМНАЯ БОМБА ДОЛЖНА БЫЛА СТАТЬ (И СТАЛА) КОПИЕЙ АМЕРИКАНСКОЙ. НО ДЕЛО НЕ ОГРАНИЧИВАЛОСЬ ПРОСТЫМ ВОСПРОИЗВОДСТВОМ ЗАРУБЕЖНЫХ НАРАБОТОК. РУКОВОДСТВО АТОМНОГО ПРОЕКТА ПРЕКРАСНО ПОНИМАЛО, ЧТО ПОСТУПЛЕНИЕ РАЗВЕДИНФОРМАЦИИ МОЖЕТ ПРЕКРАТИТЬСЯ В ЛЮБОЙ МОМЕНТ. ПОЭТОМУ КОПИРОВАНИЕ АМЕРИКАНСКОГО ОБРАЗЦА РАССМАТРИВАЛОСЬ КАК ОТПРАВНОЙ ШАГ В СОВЕРШЕНСТВОВАНИИ « ЗАИМСТВОВАННЫХ» ТЕХНОЛОГИЙ. ТАКИМ ОБРАЗОМ РАССЧИТЫВАЛИ ПЕРЕЙТИ ОТ ДОГОНЯЮЩЕЙ», « ИМИТАЦИОННОЙ» МОДЕЛИ РАЗВИТИЯ ЯДЕРНО-ОРУЖЕЙНОГО КОМПЛЕКСА К МОДЕЛИ « ИННОВАЦИОННОЙ». ИДЕЯ СОЗДАНИЯ ПЕРВОЙ АТОМНОЙ БОМБЫ ПО ЗАРУБЕЖНЫМ «ЛЕКАЛАМ» С ПОНИМАНИЕМ ВОСПРИНИМАЛАСЬ ЕЕ ГЛАВНЫМИ РАЗРАБОТЧИКАМИ. ЕДИНСТВЕННОЕ, ПОЖАЛУЙ, ИСКЛЮЧЕНИЕ СОСТАВЛЯЛ П.Л. КАПИЦА, ПРИВЛЕЧЕННЫЙ К РАБОТЕ В АТОМНОМ ПРОЕКТЕ. ОН СЧИТАЛ, ЧТО НУЖНО ИДТИ СВОИМ ПУТЕМ», А НЕ КОПИРОВАТЬ АМЕРИКАНЦЕВ. ЕСЛИ СТРЕМИТЬСЯ «ПЕРЕПРОБОВАТЬ» ВСЕ, ЧТО ОНИ СДЕЛАЛИ, ТО ВЫЙДЕТ «ДОЛГО И ДОРОГО». ПОВТОРЯЯ ПУТЬ ВПЕРЕДИ ИДУЩЕГО, НЕЛЬЗЯ НЕ ТОЛЬКО « ПЕРЕГНАТЬ», НО ДАЖЕ И «ДОГНАТЬ» ЕГО, УТВЕРЖДАЛ УЧЕНЫЙ. СЛЕДОВАТЕЛЬНО, ПОЛАГАЛ П.Л. КАПИЦА, СНАЧАЛА НУЖНО «ВЫРАБОТАТЬ ДВУХЛЕТНИЙ ПЛАН ПОДГОТОВКИ ПРОМЫШЛЕННОСТИ» К РЕШЕНИЮ АТОМНОЙ ПРОБЛЕМЫ, А ЗА ЭТО ВРЕМЯ « НАЛАДИТЬ» ЕЕ « НАУЧНУЮ ЧАСТЬ» И УЖЕ ЗАТЕМ, « НАЙДЯ НОВЫЕ ПУТИ», НАВЕРСТАТЬ ОТСТАВАНИЕ ОТ АМЕРИКАНЦЕВ. ГЛАВНУЮ РОЛЬ В АТОМНОМ ПРОЕКТЕ, ПО МНЕНИЮ АКАДЕМИКА, ДОЛЖЕН БЫЛ ИГРАТЬ НАУЧНЫЙ КОМИССАР» : ЕМУ СЛЕДОВАЛО ПЕРЕДАТЬ ПРАВО СОГЛАСОВЫВАТЬ - « СКРЕПЛЯТЬ» - «ВСЯКИЕ ПРОТОКОЛЫ» И « ПРИКАЗЫ РАЗНЫХ НАЧАЛЬНИКОВ». В ТАКОМ КАЧЕСТВЕ П.Л. КАПИЦА, СУДЯ ПО ВСЕМУ, ВИДЕЛ СЕБЯ, В ПРОТИВНОМ СЛУЧАЕ ОН НЕ СЧИТАЛ ВОЗМОЖНЫМ СВОЕ ДАЛЬНЕЙШЕЕ УЧАСТИЕ В АТОМНОМ ПРОЕКТЕ. ПО ЕГО МНЕНИЮ, « УЧЕНЫЙ С МИРОВЫМ ИМЕНЕМ» НЕ МОЖЕТ СЕБЕ ПОЗВОЛИТЬ «БЫТЬ СЛЕПЫМ ИСПОЛНИТЕЛЕМ», ОСОБЕННО В ДЕЛЕ, КОТОРОЕ « ВЫРОЖДАЕТСЯ». ${ }^{25}$

ПРЕДЛОЖЕНИЯ П.Л. КАПИЦЫ НЕ НАШЛИ ПОДДЕРЖКИ У АДМИНИСТРАТИВНОГО И НАУЧНОГО РУКОВОДСТВА АТОМНЫМ ПРОЕКТОМ : С ОДНОЙ СТОРОНЫ, ПРЕДЛАГАЕМЫЕ ИМ ПУТИ РЕШЕНИЯ ПРОБЛЕМЫ ОТНОСИЛИ ДОСТИЖЕНИЕ КОНКРЕТНОЙ ЦЕЛИ - СОЗДАНИЕ АТОМНОЙ БОМБЫ - В НЕОПРЕДЕЛЕННОЕ БУДУЩЕЕ ; С ДРУГОЙ - ПРЕТЕНЗИИ П.Л. КАПИЦЫ НА ОСОБУЮ РОЛЬ В АТОМНОМ ПРОЕКТЕ ВЫЗЫВАЛИ РАСТУЩЕЕ НЕПРИЯТИЕ. 
В ОТСТАИВАНИИ СВОИХ ВЗГЛЯДОВ АКАДЕМИК АПЕЛЛИРОВАЛ К И.В. СТАЛИНУ, НО ТАКЖЕ БЕЗРЕЗУЛЬТАТНО. В ДЕКАБРЕ 1945 Г. СНК СССР СПЕЦИАЛЬНЫМ ПОСТАНОВЛЕНИЕМ « УДОВЛЕТВОРИЛ ПРОСЬБУ» П.Л. КАПИЦЫ ОБ ЕГО ОСВОБОЖДЕНИИ ОТ РАБОТЫ В АТОМНОМ ПРОЕКТЕ ${ }^{26}$. ТАКИМ ОБРАЗОМ, В ДИСКУССИИ О ПУТЯХ СОЗДАНИЯ АТОМНОЙ БОМБЫ БЫЛА ПОСТАВЛЕНА ТОЧКА: ОСВОЕНИЕ НОВЕЙШИХ ЗАРУБЕЖНЫХ ДОСТИЖЕНИЙ ПРИЗНАЛИ НЕОБХОДИМЫМ УСЛОВИЕМ РЕШЕНИЯ АТОМНОЙ ПРОБЛЕМЫ.

И ЭТА СТРАТЕГИЯ СЕБЯ ОПРАВДАЛА, ЕСЛИ СУДИТЬ С ПОЗИЦИЙ ВЫСШЕГО РУКОВОДСТВА СТРАНЫ. ОПЫТНЫЙ ЯДЕРНЫЙ ЗАРЯД ИСПЫТАЛИ ГОРАЗДО РАНЬШЕ, ЧЕМ ОЖИДАЛИ ВСЕ ИНОСТРАННЫЕ АНАЛИТИКИ И ПОЛИТИКИ ${ }^{27}$. В ГЛАЗАХ СОВЕТСКИХ ЛИДЕРОВ ТАКОЙ РЕЗУЛЬТАТ ПЕРЕКРЫВАЛ ЛЮБЫЕ ИЗДЕРЖКИ. ОДНОВРЕМЕННО, ОН ОТКРЫВАЛ ДОРОГУ ДЛЯ РЕАЛИЗАЦИИ СОБСТВЕННЫХ ИДЕЙ ПРИ ДОРАБОТКЕ « ЗАИМСТВОВАННЫХ» ТЕХНОЛОГИЙ, НАУЧНЫХ И КОНСТРУКТОРСКИХ РЕШЕНИЙ. ПЕРВЫЕ ШАГИ В ЭТОМ НАПРАВЛЕНИИ БЫЛИ СДЕЛАНЫ ЕЩЕ ДО ИСПЫТАНИЯ АМЕРИКАНСКОЙ « КОПИИ» АТОМНОЙ БОМБЫ. ЗНАЧИМОГО РЕЗУЛЬТАТА УДАЛОСЬ ДОБИТЬСЯ В СОЗДАНИИ ЯДЕРНЫХ БОЕЗАРЯДОВ. ИХ УСОВЕРШЕНСТВОВАННЫЕ ОБРАЗЦЫ ИСПЫТАЛИ В 1951 И 1953 ГГ. ПО СВОИМ ХАРАКТЕРИСТИКАМ ОНИ ЗАМЕТНО ПРЕВОСХОДИЛИ ПЕРВЫЕ АМЕРИКАНСКИЕ « ИЗДЕЛИЯ» И ИХ СОВЕТСКИЙ АНАЛОГ. ЭТО ПОЗВОЛИЛО ЗАПУСТИТЬ СЕРИЙНОЕ ПРОИЗВОДСТВО АВИАЦИОННЫХ АТОМНЫХ БОМБ ДЛЯ ТЯЖЕЛЫХ И СРЕДНИХ БОМБАРДИРОВЩИКОВ, НАЧАТЬ ПРОЕКТИРОВАНИЕ ЯДЕРНЫХ ЗАРЯДОВ ДЛЯ МОРСКИХ ТОРПЕД, КРЫЛАТЫХ И БАЛЛИСТИЧЕСКИХ РАКЕТ, СТВОЛЬНОЙ АРТИЛЛЕРИИ. В 1953 Г. СОВЕТСКИЕ УЧЕНЫЕ И КОНСТРУКТОРЫ ВПЕРВЫЕ В МИРЕ ПРЕДЛОЖИЛИ ПРИЕМЛЕМУЮ ДЛЯ ПРОМЫШЛЕННОСТИ ЦЕНТРИФУЖНУЮ ТЕХНОЛОГИЮ ОБОГАЩЕНИЯ УРАНА. ЕЕ ПРИМЕНЕНИЕ ПОЗВОЛЯЛО РАДИКАЛЬНО НАРАСТИТЬ ПРОИЗВОДСТВО ДЕЛЯЩИХСЯ МАТЕРИАЛОВ КАК ДЛЯ ВОЕННЫХ ЦЕЛЕЙ, ТАК И ДЛЯ АТОМНОЙ ЭНЕРГЕТИКИ ${ }^{28}$. СОВЕРШЕННО ОРИГИНАЛЬНОЙ ОКАЗАЛАСЬ КОНСТРУКЦИЯ ЗАРЯДОВ НОВОГО, ТЕРМОЯДЕРНОГО, КЛАССА (ТАК НАЗЫВАЕМОЙ СЛОЙКИ И ДВУХСТАДИЙНОГО ЗАРЯДА). ИХ ИСПЫТАНИЯ СОСТОЯЛИСЬ В 1953 И 1955 ГГ., ЧТО ПОДТВЕРДИЛО СПОСОБНОСТЬ СОВЕТСКОГО СОЮЗА САМОСТОЯТЕЛЬНО РАЗРАБАТЫВАТЬ НОВЫЕ СИСТЕМЫ ЯДЕРНОГО ОРУЖИЯ ${ }^{29}$. ЭТО БЫЛО ОСОБЕННО ВАЖНО В СВЯЗИ С СОКРАЩЕНИЕМ ПРИТОКА РАЗВЕДДАННЫХ. НО ЧТОБЫ ДОБИТЬСЯ ТАКОГО РЕЗУЛЬТАТА, ПОНАДОБИЛОСЬ НАПРЯЧЬ СИЛЫ ВСЕЙ СТРАНЫ. ДЛЯ СОВЕТСКОГО РУКОВОДСТВА ЗДЕСЬ НЕ БЫЛО НИКАКИХ ОГРАНИЧЕНИЙ. ПРОБЛЕМА ЗАКЛЮЧАЛАСЬ В ОДНОМ : КАК ПОСТАВИТЬ ДЕЛО, ЧТОБЫ АМБИЦИОЗНЫЕ ЦЕЛИ РУКОВОДИТЕЛЕЙ СССР СТАЛИ РЕАЛЬНОСТЬЮ. ИНАЧЕ ГОВОРЯ, В РАМКАХ КОМАНДНОЙ ЭКОНОМИКИ ТРЕБОВАЛОСЬ НАЙТИ АДЕКВАТНЫЕ ЗАДАЧАМ АТОМНОГО ПРОЕКТА УПРАВЛЕНЧЕСКИЕ МЕХАНИЗМЫ.

\section{Структура управления}

ОТВЕТСТВЕННОСТЬ ЗА ОСУЩЕСТВЛЕНИЕ АТОМНОГО ПРОЕКТА ВОЗЛОЖИЛИ НА СПЕЦИАЛЬНО СОЗДАННУЮ ИЕРАРХИЧЕСКУЮ СТРУКТУРУ. ОНА ЗАНЯЛА ОСОБОЕ МЕСТО В СИСТЕМЕ ВЛАСТНЫХ ОТНОШЕНИЙ ПОЗДНЕГО СТАЛИНИЗМА. ИХ ОТЛИЧИТЕЛЬНОЙ ОСОБЕННОСТЬЮ ЯВЛЯЛОСЬ СОСРЕДОТОЧЕНИЕ «ВОПРОСОВ РАЗВИТИЯ» ПРОМЫШЛЕННОСТИ, ТРАНСПОРТА, ТОРГОВЛИ, СОЦИАЛЬНОЙ ИНФРАСТРУКТУРЫ, ОРГАНИЗАЦИИ СЕЛЬСКОХОЗЯЙСТВЕННОГО ПРОИЗВОДСТВА В КОМПЕТЕНЦИИ ПРАВИТЕЛЬСТВА. ОНО ЖЕ ОТВЕЧАЛО ЗА ПЛАНИРОВАНИЕ И ФИНАНСИРОВАНИЕ НАРОДНОГО ХОЗЯЙСТВА, ЗА ДЕНЕЖНОЕ ОБРАЩЕНИЕ И СНАБЖЕНИЕ, ЗА ТЕХНИЧЕСКИЙ ПРОГРЕСС И РАСПРЕДЕЛЕНИЕ ТРУДОВЫХ РЕСУРСОВ. ПАРТИЙНЫЕ ОРГАНЫ В ЛИЦЕ ОРГБЮРО, 
СЕКРЕТАРИАТА, ОТДЕЛОВ ЦК, ОБЛАСТНЫХ КОМИТЕТОВ И ДАЖЕ ПОЛИТБЮРО НЕ ВМЕШИВАЛИСЬ В ЭТИ ПРОЦЕССЫ ${ }^{30}$. ФУНКЦИИ ПРАВИТЕЛЬСТВА ФАКТИЧЕСКИ ВЫПОЛНЯЛО БЮРО (ПРЕЗИДИУМ) СОВЕТА МИНИСТРОВ СССР. ЕМУ НЕПОСРЕДСТВЕННО ПОДЧИНЯЛИСЬ ОБЩЕЭКОНОМИЧЕСКИЕ ВЕДОМСТВА : ГОСПЛАН, МИНФИН, ГОССНАБ, ГОСТЕХНИКА И ДР. И ХОЗЯЙСТВЕННЫЕ МИНИСТЕРСТВА. ПОСЛЕДНИЕ РУКОВОДИЛИ СЛОЖИВШИМИСЯ ОТРАСЛЕВЫМИ КОМПЛЕКСАМИ. ИНОГДА ИХ СОЗДАВАЛИ ДЛЯ РЕШЕНИЯ КАКИХ-ТО ПРИОРИТЕТНЫХ ЗАДАЧ. ТОГДА ОРГАНИЗАЦИЯ МИНИСТЕРСТВ ОПЕРЕЖАЛА ПРОЦЕСС РЕАЛЬНОГО ФОРМИРОВАНИЯ ОТРАСЛЕЙ НАРОДНОГО ХОЗЯЙСТВА И ВЫСТУПАЛА В КАЧЕСТВЕ МОЩНОГО СТИМУЛА ИХ УСКОРЕННОГО СТАНОВЛЕНИЯ. НО В ЛЮБОМ СЛУЧАЕ МИНИСТЕРСТВА ИМЕЛИ АНАЛОГИЧНУЮ СТРУКТУРУ. ИХ ЯДРО СОСТАВЛЯЛИ ПРЕДПРИЯТИЯ ОТРАСЛЕВОЙ СПЕЦИАЛИЗАЦИИ. ТЕОРЕТИЧЕСКИ ВСЕ НЕОБХОДИМОЕ ДЛЯ СВОЕГО НОРМАЛЬНОГО ФУНКЦИОНИРОВАНИЯ ОНИ ДОЛЖНЫ БЫЛИ ПОЛУЧАТЬ В РАМКАХ МЕЖОТРАСЛЕВОЙ КООПЕРАЦИИ. ОДНАКО НА ПРАКТИКЕ ЭТО НЕ ВСЕГДА ПОЛУЧАЛОСЬ. АДМИНИСТРАТИВНЫЕ БАРЬЕРЫ ДЕЛАЛИ НЕНАДЕЖНЫМИ ПОСТАВКИ ТАК НАЗЫВАЕМЫХ СМЕЖНИКОВ. В РЕЗУЛЬТАТЕ МИНИСТЕРСТВА БЫЛИ ВЫНУЖДЕНЫ РАЗВИВАТЬ ПРОИЗВОДСТВА, НЕ ОТНОСЯЩИЕСЯ К ИХ ПРОФИЛЮ. ТАКОЕ СТРЕМЛЕНИЕ К ХОЗЯЙСТВЕННОЙ АВТОНОМИИ ОБОРАЧИВАЛОСЬ ФОРМИРОВАНИЕМ ОТРАСЛЕВЫХ «УДЕЛЬНЫХ КНЯЖЕСТВ». И ТРЕБОВАЛСЯ СИЛЬНЫЙ АДМИНИСТРАТИВНЫЙ НАЖИМ, ЧТОБЫ ОБЕСПЕЧИТЬ МЕЖОТРАСЛЕВОЕ ВЗАИМОДЕЙСТВИЕ ${ }^{31}$.

ЭТА ПРОБЛЕМА ОБОЗНАЧИЛАСЬ УЖЕ НА НАЧАЛЬНОЙ СТАДИИ АТОМНОГО ПРОЕКТА. СТАЛО ОЧЕВИДНО, ЧТО К ЕГО РЕАЛИЗАЦИИ НУЖНО ПРИВЛЕЧЬ ОГРОМНОЕ ЧИСЛО НАУЧНО-ИССЛЕДОВАТЕЛЬСКИХ И ПРОЕКТНО-КОНСТРУКТОРСКИХ ОРГАНИЗАЦИЙ, ПРОМЫШЛЕННЫХ ПРЕДПРИЯТИЙ РАЗЛИЧНОГО ОТРАСЛЕВОГО ПРОФИЛЯ. НО ЗДЕСЬ СРАЗУ ВОЗНИКАЛ ВОПРОС : КАК ОБЕСПЕЧИТЬ ДОЛЖНОЕ РУКОВОДСТВО ИМИ? СТАНДАРТНЫЙ ВАРИАНТ - СОЗДАТЬ СПЕЦИАЛИЗИРОВАННОЕ МИНИСТЕРСТВО И ПЕРЕПОДЧИНИТЬ ЕМУ НЕОБХОДИМЫЕ ПРОИЗВОДСТВЕННЫЕ СТРУКТУРЫ - НЕ ПОДХОДИЛ. ЯДЕРНО-ОРУЖЕЙНЫЙ КОМПЛЕКС СОЗДАВАЛСЯ « С НУЛЯ», И НЕ БЫЛО ПОЛНОЙ ЯСНОСТИ, КАКИЕ ПРЕДПРИЯТИЯ И В КАКОМ ОБЪЕМЕ НУЖНО ПРИВЛЕЧЬ, ЧТОБЫ ОБЕСПЕЧИТЬ ЕГО ФУНКЦИОНИРОВАНИЕ. ТАКЖЕ ПОДЖИМАЛИ СРОКИ, А ДЛЯ ПЕРЕПОДЧИНЕНИЯ И ПЕРЕПРОФИЛИРОВАНИЯ ДЕЙСТВУЮЩИХ ПРОИЗВОДСТВ ТРЕБОВАЛОСЬ ДЛИТЕЛЬНОЕ ВРЕМЯ. МОЖНО ПОЛАГАТЬ, ЧТО ЭТО НИКАК НЕ УСТРАИВАЛО ВЫСШЕЕ РУКОВОДСТВО СТРАНЫ. ВЫХОД НАШЛИ В ИСПОЛЬЗОВАНИИ НЕСТАНДАРТНОЙ СХЕМЫ ОРГАНИЗАЦИИ РАБОТЫ. ИСПОЛНИТЕЛЬНОМУ ОРГАНУ АТОМНОГО ПРОЕКТА РЕШИЛИ НЕПОСРЕДСТВЕННО ПОДЧИНИТЬ ЛИШЬ ВНОВЬ СОЗДАЮЩИЕСЯ ПРЕДПРИЯТИЯ: ПО ДОБЫЧЕ УРАНОВОЙ РУДЫ И ЕЕ ПЕРЕРАБОТКЕ, ПО ПОЛУЧЕНИЮ ЯДЕРНОЙ ВЗРЫВЧАТКИ, КОНСТРУИРОВАНИЮ И ИЗГОТОВЛЕНИЮ АТОМНОЙ БОМБЫ. ВСЕ ОСТАЛЬНЫЕ НУЖДЫ АТОМНОГО ПРОЕКТА ДОЛЖНЫ БЫЛИ УДОВЛЕТВОРЯТЬ «СМЕЖНИКИ». ЧТОБЫ ЭТА СХЕМА РАБОТАЛА, РУКОВОДСТВО АТОМНОГО ПРОЕКТА ПОЛУЧАЛО ПРАВО САМОСТОЯТЕЛЬНО ПЛАНИРОВАТЬ СВОЮ ДЕЯТЕЛЬНОСТЬ И ДАВАТЬ УКАЗАНИЯ ЛЮБЫМ ОРГАНАМ ХОЗЯЙСТВЕННОГО УПРАВЛЕНИЯ, ОТДЕЛЬНЫМ ПРЕДПРИЯТИЯМ И ОРГАНИЗАЦИЯМ. ДЛЯ КОНТРОЛЯ ЗА «НЕУКОСНИТЕЛЬНЫМ» ИСПОЛНЕНИЕМ ПРИНЯТЫХ РЕШЕНИЙ БЫЛА СОЗДАНА ЖЕСТКАЯ УПРАВЛЕНЧЕСКАЯ ВЕРТИКАЛЬ.

НА САМОМ ВЕРХУ УПРАВЛЕНЧЕСКОЙ ПИРАМИДЫ НАХОДИЛСЯ СПЕЦИАЛЬНЫЙ КОМИТЕТ ПРИ ГОСУДАРСТВЕННОМ КОМИТЕТЕ ОБОРОНЫ (ПОСЛЕ УПРАЗДНЕНИЯ ПОСЛЕДНЕГО ДЕЙСТВОВАЛ ПРИ СНК СССР, А ЗАТЕМ - ПРИ СОВЕТЕ МИНИСТРОВ СССР). ЕГО ПРЕДСЕДАТЕЛЕМ НАЗНАЧИЛИ Л.П.БЕРИЮ. КАК ПРАВИТЕЛЬСТВЕННЫЙ ОРГАН, ОН, 
ОСВОБОЖДАЛСЯ ОТ ПАРТИЙНОГО КОНТРОЛЯ. НЕ ВМЕШИВАЛИСЬ В ЕГО ДЕЛА И КАКИЕ-ЛИБО ГОСУДАРСТВЕННЫЕ СТРУКТУРЫ. С САМОГО НАЧАЛА СВОЕЙ ДЕЯТЕЛЬНОСТИ СПЕЦКОМИТЕТ «ВНОСИЛ» ПРЕДЛОЖЕНИЯ «НЕПОСРЕДСТВЕННО НА УТВЕРЖДЕНИЕ ПРЕДСЕДАТЕЛЯ ГОКО», Т.Е. И.В.СТАЛИНА. ОН ИМЕЛ ПРАВО ПРИВЛЕКАТЬ К РАБОТЕ ЛЮБЫХ ИСПОЛНИТЕЛЕЙ, НЕЗАВИСИМО ОТ ИХ ВЕДОМСТВЕННОЙ ПРИНАДЛЕЖНОСТИ. ДАЛЬНЕЙШЕЕ РАСШИРЕНИЕ ЕГО КОМПЕТЕНЦИИ ПРОИЗОШЛО В ДЕКАБРЕ 1949 Г. - МАЕ 1950 Г., ВСКОРЕ ПОСЛЕ ИСПЫТАНИЯ ПЕРВОЙ СОВЕТСКОЙ АТОМНОЙ БОМБЫ. УТВЕРЖДЕННЫЕ СПЕЦКОМИТЕТОМ ПРОЕКТЫ «СПЕЦИАЛЬНЫХ РАБОТ» СТАЛИ ПРИНИМАТЬСЯ К ИСПОЛНЕНИЮ ГОСПЛАНОМ, ГОССНАБОМ, ГОСТЕХНИКОЙ И МИНФИНОМ БЕЗ ВСЯКИХ ДАЖЕ ФОРМАЛЬНЫХ ОБОСНОВАНИЙ. ${ }^{32}$ ЭТО, ПО СУТИ, ЗАВЕРШИЛО ПРЕВРАЩЕНИЕ ЯДЕРНО-ОРУЖЕЙНОГО КОМПЛЕКСА В СВОЕГО РОДА ГОСУДАРСТВО В ГОСУДАРСТВЕ.

СПЕЦИАЛЬНЫЙ КОМИТЕТ ДЕЙСТВОВАЛ ЧЕРЕЗ ПОДОТЧЕТНЫЕ ТОЛЬКО ЕМУ СТРУКТУРЫ. НЕПОСРЕДСТВЕННО ПРЕДСЕДАТЕЛЮ СПЕЦКОМИТЕТА ПОДЧИНЯЛОСЬ БЮРО № 2. В НЕГО ВХОДИЛИ КАК ОПЕРАТИВНЫЕ РАБОТНИКИ СПЕЦСЛУЖБ, ТАК И НАУЧНЫЕ СОТРУДНИКИ. ОНО ЗАНИМАЛОСЬ ПЕРЕВОДОМ И ОБРАБОТКОЙ МАТЕРИАЛОВ, ПОСТУПАВШИХ ПО ЛИНИИ РАЗВЕДКИ. ДЛЯ «ПРЕДВАРИТЕЛЬНОГО РАССМОТРЕНИЯ НАУЧНЫХ И ТЕХНИЧЕСКИХ ВОПРОСОВ» БЫЛ СОЗДАН ТЕХНИЧЕСКИЙ СОВЕТ ${ }^{33}$. ЗАТЕМ ОРГАНИЗОВАЛИ ИНЖЕНЕРНО-ТЕХНИЧЕСКИЙ СОВЕТ - ДЛЯ « ОБЕСПЕЧЕНИЯ... РУКОВОДСТВА ПРОЕКТИРОВАНИЕМ И СООРУЖЕНИЕМ ПРЕДПРИЯТИЙ» ФОРМИРУЮЩЕЙСЯ ОТРАСЛИ. ОДНАКО РАЗДЕЛЬНОЕ «РАССМОТРЕНИЕ» НАУЧНЫХ И ИНЖЕНЕРНЫХ ВОПРОСОВ ВЕЛО К РАЗЛИЧНОГО РОДА НЕСТЫКОВКАМ, УТРАТЕ КОМПЛЕКСНОГО ПОДХОДА К РЕШЕНИЮ МНОЖАЩИХСЯ ПРОБЛЕМ. ПОЭТОМУ В АПРЕЛЕ 1946 Г. ОБА СОВЕТА ОБЪЕДИНИЛИ В ОДНУ СТРУКТУРУ - НАУЧНО-ТЕХНИЧЕСКИЙ СОВЕТ ${ }^{34}$, СТАВШИЙ МОЗГОВЫМ ЦЕНТРОМ АТОМНОГО ПРОЕКТА. СОВЕТ НЕ ОБЛАДАЛ РАСПОРЯДИТЕЛЬНЫМИ ПОЛНОМОЧИЯМИ, НО ИМЕННО ОН ОПРЕДЕЛЯЛ, ЧТО, КАК И КОГДА ДЕЛАТЬ. ДО ДЕКАБРЯ 1949 Г. СОВЕТ ВОЗГЛАВЛЯЛ Б.Л. ВАННИКОВ. ЗАТЕМ ЕГО ПРЕДСЕДАТЕЛЕМ НАЗНАЧИЛИ И.В. КУРЧАТОВА, БЫВШЕГО ДО ЭТОГО ЗАМЕСТИТЕЛЕМ ВАННИКОВА.

21 В ДЕКАБРЕ 1946 Г. ПО РЕШЕНИЮ СПЕЦКОМИТЕТА БЫЛ СОЗДАН ЕЩЕ ОДИН ЭКСПЕРТНО-СОВЕЩАТЕЛЬНЫЙ ОРГАН - УЧЕНЫЙ СОВЕТ ПРИ ПРЕЗИДЕНТЕ АКАДЕМИИ НАУК СССР, КОТОРЫЙ ПО СОГЛАСОВАНИЮ С НАУЧНО-ТЕХНИЧЕСКИМ СОВЕТОМ НАПРАВЛЯЛ ЯДЕРНО-ФИЗИЧЕСКИЕ ИССЛЕДОВАНИЯ В «ОТКРЫТЫХ» ОБЛАСТЯХ НАУКИ, ТЕХНИКИ, МЕДИЦИНЫ ${ }^{35}$.

ИСПОЛНИТЕЛЬНЫМ ОРГАНОМ В АТОМНОМ ПРОЕКТЕ ЯВЛЯЛОСЬ ПЕРВОЕ ГЛАВНОЕ УПРАВЛЕНИЕ (ПГУ) ПРИ СНК (ПОЗДНЕЕ - СОВЕТЕ МИНИСТРОВ) СССР. НАЧАЛЬНИКОМ ЕГО СТАЛ КРУПНЫЙ ХОЗЯЙСТВЕННИК Б.Л. ВАННИКОВ. ПО СВОЕМУ СТАТУСУ ПГУ ПРИРАВНИВАЛОСЬ К СОЮЗНОМУ МИНИСТЕРСТВУ, НО ОНО БЫЛО НЕ СОВСЕМ ОБЫЧНЫМ ВЕДОМСТВОМ. ОСЕНЬЮ 1945 Г. ЕМУ ИЗ СИСТЕМЫ НКВД ПЕРЕДАЛИ ПРЕДПРИЯТИЯ И ОРГАНИЗАЦИИ, СВЯЗАННЫЕ С ДОБЫЧЕЙ И ПЕРЕРАБОТКОЙ УРАНОВЫХ РУД, РАЗРАБОТКОЙ СООТВЕТСТВУЮЩИХ ТЕХНОЛОГИЙ. В СОСТАВ ПГУ ТАКЖЕ ВОШЛИ ЗАВОДЫ, ЗАНИМАВШИЕСЯ ПОЛУЧЕНИЕМ МЕТАЛЛИЧЕСКОГО УРАНА, ПРОИЗВОДСТВОМ СПЕЦИАЛЬНОГО ГОРНОРУДНОГО И ХИМИКО-ТЕХНОЛОГИЧЕСКОГО ОБОРУДОВАНИЯ. ЗАТЕМ РАЗВЕРНУЛОСЬ СТРОИТЕЛЬСТВО КОМБИНАТОВ ПО ОБОГАЩЕНИЮ УРАНА, ПОЛУЧЕНИЮ ПЛУТОНИЯ, КОНСТРУИРОВАНИЮ И ИЗГОТОВЛЕНИЮ ЯДЕРНЫХ БОЕЗАРЯДОВ. ОДНАКО В ЦЕЛОМ, В НЕПОСРЕДСТВЕННОМ ПОДЧИНЕНИИ ПГУ НАХОДИЛОСЬ ОГРАНИЧЕННОЕ ЧИСЛО 
ИСПОЛНИТЕЛЕЙ. В ТО ЖЕ ВРЕМЯ ПО ЕГО ЗАДАНИЯМ РАБОТАЛИ ДЕСЯТКИ НАУЧНО-ИССЛЕДОВАТЕЛЬСКИХ И ПРОЕКТНЫХ ИНСТИТУТОВ, КОНСТРУКТОРСКИХ БЮРО, ПРОМЫШЛЕННЫХ ПРЕДПРИЯТИЙ И СТРОИТЕЛЬНЫХ ОРГАНИЗАЦИЙ РАЗЛИЧНОЙ ВЕДОМСТВЕННОЙ ПРИНАДЛЕЖНОСТИ. ВСКОРЕ КОЛИЧЕСТВО ПРИВЛЕКАЕМЫХ НА ПЛАНОВОЙ ОСНОВЕ « СМЕЖНИКОВ» ПРЕВЫСИЛО СТО ЕДИНИЦ.

ОСОБОЕ МЕСТО В СТРУКТУРЕ АТОМНОГО ПРОЕКТА ЗАНЯЛИ ОРГАНЫ УПРАВЛЕНИЯ УРАНОДОБЫВАЮЩЕЙ ПРОМЫШЛЕННОСТИ ЕВРОПЕЙСКИХ СТРАН. ЭТО БЫЛИ СОВЕТСКО-ЧЕХОСЛОВАЦКОЕ И СОВЕТСКО-БОЛГАРСКОЕ ГОРНЫЕ ОБЩЕСТВА, ПРЕДПРИЯТИЕ « КУЗНЕЦКИЕ РУДНИКИ» В ПОЛЬШЕ, СОВЕТСКОЕ АКЦИОНЕРНОЕ ОБЩЕСТВО «ВИСМУТ» В ВОСТОЧНОЙ ГЕРМАНИИ (ГДР). ОНИ ДЕЙСТВОВАЛИ НА ОСНОВЕ МЕЖПРАВИТЕЛЬСТВЕННЫХ СОГЛАШЕНИЙ, НО ФАКТИЧЕСКИ ЯВЛЯЛИСЬ ФИЛИАЛАМИ ПЕРВОГО ГЛАВНОГО УПРАВЛЕНИЯ. ИХ РОЛЬ В АТОМНОМ ПРОЕКТЕ ТРУДНО ПЕРЕОЦЕНИТЬ. ТАК, В НАЧАЛЕ 1950-Х ГГ. ВОСТОЧНОЕВРОПЕЙСКИЕ ПРЕДПРИЯТИЯ НА $80 \%$ ОБЕСПЕЧИВАЛИ ЕГО ПОТРЕБНОСТИ В УРАНОСОДЕРЖАЩЕМ СЫРЬЕ, ПРИЧЕМ НА ДОЛЮ ГДР ПРИХОДИЛОСЬ ДО ДВУХ ТРЕТЕЙ ВСЕХ ПОСТАВОК ${ }^{36}$.

В ДЕКАБРЕ 1949 Г. ПРОИЗОШЛА РЕОРГАНИЗАЦИЯ ПЕРВОГО ГЛАВНОГО УПРАВЛЕНИЯ : ИЗ НЕГО « ВЫДЕЛИЛИ» ГОРНОДОБЫВАЮЩИЙ КОМПЛЕКС. ДЛЯ УПРАВЛЕНИЯ ИМ СОЗДАЛИ ВТОРОЕ ГЛАВНОЕ УПРАВЛЕНИЕ ПРИ СОВЕТЕ МИНИСТРОВ СССР, ТАКЖЕ ПОДВЕДОМСТВЕННОЕ СПЕЦКОМИТЕТУ ${ }^{37}$. ПРОВЕДЕННАЯ ПЕРЕСТРОЙКА НОСИЛА РУТИННЫЙ ХАРАКТЕР И НИЧЕГО ПРИНЦИПИАЛЬНО НЕ ИЗМЕНИЛА В РУКОВОДСТВЕ АТОМНЫМ ПРОЕКТОМ. В НАЧАЛЕ 1950-ХГГ. КОЛИЧЕСТВО РАБОТНИКОВ В ОРГАНИЗАЦИЯХ И НА ПРЕДПРИЯТИЯХ ПЕРВОГО И ВТОРОГО ГЛАВНЫХ УПРАВЛЕНИЙ ДОСТИГЛО 120 ТЫС. ЕЩЕ 190 ТЫС. ЧЕЛОВЕК БЫЛО ЗАНЯТО НА УРАНОВЫХ РУДНИКАХ И ОБОГАТИТЕЛЬНЫХ ПРЕДПРИЯТИЯХ В ГЕРМАНИИ, ЧЕХОСЛОВАКИИ, ПОЛЬШИ, БОЛГАРИИ. ДОСТУПНЫЕ ДАННЫЕ НЕ ПОЗВОЛЯЮТ ОЦЕНИТЬ ЧИСЛЕННОСТЬ ПЕРСОНАЛА ОРГАНИЗАЦИЙ-СМЕЖНИКОВ, НО ОЧЕВИДНО, ЧТО ОН НАСЧИТЫВАЛ НЕСКОЛЬКО СОТ ТЫСЯЧ ЧЕЛОВЕК. ТОЛЬКО В ГЛАВПРОМСТРОЕ МВД, ВОЗВОДИВШЕМ ОБЪЕКТЫ АТОМНОЙ ИНДУСТРИИ, РАБОТАЛО ОКОЛО 260 ТЫС. СТРОИТЕЛЕЙ ${ }^{38}$. ВСЯ ДЕЯТЕЛЬНОСТЬ ЭТОЙ ОГРОМНОЙ « ПРОИЗВОДСТВЕННОЙ АРМИИ» ЖЕСТКО НАПРАВЛЯЛАСЬ ИЗ ЕДИНОГО ЦЕНТРА.

СПЕЦИФИЧЕСКУЮ ФУНКЦИЮ В АТОМНОМ ПРОЕКТЕ ВЫПОЛНЯЛИ ПАРТИЙНЫЕ ОРГАНЫ ИНСТИТУТ ПАРТОРГОВ ЦК ВКП(Б) - КПСС И ПОЛИТОТДЕЛЫ ПРЕДПРИЯТИЙ. В СОДЕРЖАТЕЛЬНУЮ СТОРОНУ РАБОТЫ ОНИ НЕ ВМЕШИВАЛИСЬ. НА НИХ ВОЗЛАГАЛОСЬ РУКОВОДСТВО ПЕРВИЧНЫМИ ПАРТИЙНЫМИ И ОБЩЕСТВЕННЫМИ ОРГАНИЗАЦИЯМИ, ПРОВЕДЕНИЕ ИДЕОЛОГИЧЕСКОЙ И ВОСПИТАТЕЛЬНОЙ РАБОТЫ В КОЛЛЕКТИВАХ, ОСУЩЕСТВЛЕНИЕ КОНТРОЛЯ ЗА БЫТОВЫМ ПОВЕДЕНИЕМ РАБОТНИКОВ ОТРАСЛИ, УСЛОВИЯМИ ИХ ЖИЗНИ, ПОЛИТИЧЕСКИМИ НАСТРОЕНИЯМИ И Т.П. ФОРМАЛЬНО ПАРТИЙНЫЕ ОРГАНЫ ПРЕДПРИЯТИЙ И ОРГАНИЗАЦИЙ ОТРАСЛИ ПОДЧИНЯЛИСЬ ОБЛАСТНЫМ КОМИТЕТАМ ВКП(Б) - КПСС. В РЕАЛЬНОСТИ ЖЕ ИХ ДЕЯТЕЛЬНОСТЬ НАПРАВЛЯЛАСЬ РУКОВОДСТВОМ АТОМНОГО ПРОЕКТА И ПРОФИЛЬНЫМИ ОТДЕЛАМИ ЦК 39.

ОГРАНИЧЕНИЕ ПОЛНОМОЧИЙ ПАРТИЙНЫХ СТРУКТУР ВТОРОСТЕПЕННЫМИ ВОПРОСАМИ ИМЕЛО МЕСТО ВПЛОТЬ ДО СМЕРТИ И.В. СТАЛИНА И РАЗОБЛАЧЕНИЯ» Л.П. БЕРИИ. НО В ОТДЕЛЬНЫХ СЛУЧАЯХ ЦЕНТРАЛЬНЫЕ ПАРТИЙНЫЕ ОРГАНЫ ПЫТАЛИСЬ ВЫЙТИ ЗА УСТАНОВЛЕННЫЕ ИМ РАМКИ ОТВЕТСТВЕННОСТИ. ТАК БЫЛО В ХОДЕ ПОЛИТИКО-ИДЕОЛОГИЧЕСКИХ КАМПАНИЙ, РАЗВЕРНУТЫХ В СТРАНЕ В КОНЦЕ 1940-Х ГГ. ХОРОШО ИЗВЕСТНО, КАК ПЫТАЛИСЬ ОБВИНИТЬ УЧЕНЫХ, ПРИЧАСТНЫХ К РЕАЛИЗАЦИИ 
АТОМНОГО ПРОЕКТА, В ПРИВЕРЖЕННОСТИ К « БУРЖУАЗНЫМ ТЕОРИЯМ» - К КВАНТОВОЙ МЕХАНИКЕ И ТЕОРИИ ОТНОСИТЕЛЬНОСТИ. ПОПЫТКА ЭТА НЕ УВЕНЧАЛАСЬ УСПЕХОМ: ВИДИМО, СООБРАЖЕНИЯ ВОЕННО-ТЕХНИЧЕСКОГО ПОРЯДКА ПЕРЕВЕСИЛИ В ГЛАЗАХ ВЫСШЕГО РУКОВОДСТВА СТРАНЫ ПОЛИТИКО-ИДЕОЛОГИЧЕСКИЕ ВЫГОДЫ. ОДНАКО ЭТО НЕ ПОЛОЖИЛО КОНЕЦ НАПАДКАМ НА «РЕАКЦИОННОЕ ЭЙНШТЕЙНИАНСТВО». НАУЧНЫЕ ЛИДЕРЫ АТОМНОГО ПРОЕКТА БЫЛИ ВЫНУЖДЕНЫ НЕОДНОКРАТНО « ОТБИВАТЬСЯ» ОТ ОБВИНЕНИЙ В « ИДЕОЛОГИЧЕСКОЙ НЕБЛАГОНАДЕЖНОСТИ», АПЕЛЛИРУЯ К Л.П. БЕРИИ И Г.М. МАЛЕНКОВУ ${ }^{40}$.

ЦЕНТРАЛЬНЫЕ ПАРТИЙНЫЕ ОРГАНЫ ПЫТАЛИСЬ ВМЕШИВАТЬСЯ И В КАДРОВУЮ ПОЛИТИКУ НАУЧНОГО РУКОВОДСТВА АТОМНОГО ПРОЕКТА. В ХОДЕ КАМПАНИИ «ПО БОРЬБЕ С КОСМОПОЛИТИЗМОМ» ПО ИХ ПРЕДСТАВЛЕНИЮ РАЗВЕРНУЛИСЬ ПОИСКИ СРЕДИ ВЕДУЩИХ ИССЛЕДОВАТЕЛЕЙ «НЕПРИГОДНЫХ» И «НИЧЕГО НЕ СДЕЛАВШИХ». К ТАКОВЫМ В ОСНОВНОМ ОТНОСИЛИ ЛИЦ ЕВРЕЙСКОЙ НАЦИОНАЛЬНОСТИ. ОСОБЕННО ОЖЕСТОЧЕННЫМ НАПАДКАМ ПОДВЕРГСЯ АКАДЕМИК Л.Д. ЛАНДАУ. И ТОЛЬКО БЛАГОДАРЯ НАСТОЙЧИВОЙ ПРОСЬБЕ АКАДЕМИКА А.И. АЛИХАНОВА, ОБРАТИВШЕГОСЯ НЕПОСРЕДСТВЕННО К Л.П. БЕРИИ, А ТАКЖЕ ХОДАТАЙСТВУ М.Г. ПЕРВУХИНА И ГЕНЕРАЛ-МАЙОРА П.Я. МЕШИКА (ОТВЕЧАВШЕГО В АТОМНОМ ПРОЕКТЕ ЗА «ЧЕКИСТСКОЕ ОБСЛУЖИВАНИЕ») ЕГО ТОГДА ОСТАВИЛИ В ПОКОЕ ${ }^{41}$. НО ЭТИМ ДЕЛО НЕ ОГРАНИЧИЛОСЬ. В ОКТЯБРЕ 1950 Г. ОТДЕЛ АГИТАЦИИ И ПРОПАГАНДЫ ЦК ВКП(Б) ПОДГОТОВИЛ ДОКЛАДНУЮ ЗАПИСКУ О «ЗАСОРЕННОСТИ» АКАДЕМИЧЕСКИХ ИНСТИТУТОВ, РАБОТАВШИХ ПО ЗАДАНИЯМ АТОМНОГО ПРОЕКТА, « ЛЮДЬМИ НЕ ВНУШАЮЩИМИ ПОЛИТИЧЕСКОГО ДОВЕРИЯ». В ИХ ЧИСЛЕ НАЗЫВАЛИСЬ ТЕ ЖЕ ЛИЦА, ЧТО И ПОЛТОРА ГОДА НАЗАД. АКАДЕМИК Л.Д. ЛАНДАУ И ЕГО «ШКОЛА» (« 11 ДОКТОРОВ НАУК; ВСЕ ЕВРЕИ И БЕСПАРТИЙНЫЕ») ОБВИНЯЛИСЬ В « ЗАЖИМЕ» НОВЫХ НАУЧНЫХ ИДЕЙ ${ }^{42}$. ВСЕ ЖЕ ЭТИХ УЧЕНЫХ ОПЯТЬ УДАЛОСЬ ОТСТОЯТЬ. ОДНАКО НЕ ВСЕГДА ПОДОБНЫЕ ОБВИНЕНИЯ ЗАКАНЧИВАЛИСЬ СТОЛЬ БЛАГОПОЛУЧНО. СПЕЦКОМИТЕТ И ПОДКОНТРОЛЬНЫЕ ЕМУ СТРУКТУРЫ БЫЛИ ВЫНУЖДЕНЫ СЧИТАТЬСЯ С ПОЛИТИЧЕСКОЙ СИТУАЦИЕЙ И ПРИНИМАТЬ МЕРЫ, КОТОРЫЕ ОТНЮДЬ НЕ СПОСОБСТВОВАЛИ РЕШЕНИЮ СТОЯЩИХ ПЕРЕД УЧЕНЫМИ ЗАДАЧ.

ГОРАЗДО БОЛЬШУЮ РОЛЬ В УПРАВЛЕНЧЕСКОЙ ВЕРТИКАЛИ АТОМНОГО ПРОЕКТА ИГРАЛИ ТАК НАЗЫВАЕМЫЕ « УПОЛНОМОЧЕННЫЕ СОВЕТА МИНИСТРОВ СССР». В 1946-1947 ГГ. ИХ НАЗНАЧИЛИ ВО ВСЕ « ОСНОВНЫЕ ОРГАНИЗАЦИИ ПГУ» И В « ВАЖНЕЙШИЕ» ИНСТИТУТЫ, КОНСТРУКТОРСКИЕ БЮРО, НА ПРЕДПРИЯТИЯ ДРУГИХ ВЕДОМСТВ, «СВЯЗАННЫЕ С РАБОТАМИ ПО ИСПОЛЬЗОВАНИЮ АТОМНОЙ ЭНЕРГИИ». ОНИ КОНТРОЛИРОВАЛИ «СВОЕВРЕМЕННОЕ ВЫПОЛНЕНИЕ... ЗАДАНИЙ ПРАВИТЕЛЬСТВА», ОБЕСПЕЧИВАЯ СОБСТВЕННОЙ ИНФОРМАЦИЕЙ О СОСТОЯНИИ ДЕЛ РУКОВОДСТВО АТОМНОГО ПРОЕКТА. УПОЛНОМОЧЕННЫЕ ИМЕЛИ ПРАВО ИНИЦИИРОВАТЬ САНКЦИИ ЗА СРЫВ ЛЮБЫХ ЗАДАНИЙ. РУКОВОДИТЕЛИ РАЗЛИЧНОГО РАНГА ХОРОШО ЗНАЛИ, ЧЕМ ЭТО МОЖЕТ ОБЕРНУТЬСЯ, ПОЭТОМУ ОНИ ДЕЛАЛИ ВСЕ « ВОЗМОЖНОЕ И НЕВОЗМОЖНОЕ», ЧТОБЫ УПОЛНОМОЧЕННЫЕ НЕ ЗАЧИСЛИЛИ ИХ В РАЗРЯД «НЕРАДИВЫХ» ИСПОЛНИТЕЛЕЙ. ПРИ ТАКОМ ПОРЯДКЕ ВЕДОМСТВЕННЫЕ СООБРАЖЕНИЯ, ИНТЕРЕСЫ ПРЕДПРИЯТИЙ И Т. Д. ОТХОДИЛИ НА ВТОРОЙ ПЛАН. НА УПОЛНОМОЧЕННЫХ ТАКЖЕ ВОЗЛАГАЛАСЬ ОТВЕТСТВЕННОСТЬ ЗА ОХРАНУ КУРИРУЕМЫХ ИМИ ОБЪЕКТОВ И «ОГРАЖДЕНИЕ» ИХ ОТ ШПИОНАЖА, ДИВЕРСИЙ И «ПРОНИКНОВЕНИЯ ВРАЖЕСКОЙ АГЕНТУРЫ». ОНИ, КАК И ИХ СОТРУДНИКИ, ЯВЛЯЛИСЬ ОФИЦЕРАМИ СПЕЦСЛУЖБ, ЧИСЛИВШИМИСЯ В ТАК НАЗЫВАЕМОМ «ДЕЙСТВУЮЩЕМ РЕЗЕРВЕ» МИНИСТЕРСТВ ГОСБЕЗОПАСНОСТИ И ВНУТРЕННИХ ДЕЛ. УПОЛНОМОЧЕННЫЕ 
НЕПОСРЕДСТВЕННО ПОДЧИНЯЛИСЬ Л.П. БЕРИИ КАК ЗАМЕСТИТЕЛЮ ПРЕДСЕДАТЕЛЯ СОВЕТА МИНИСТРОВ СССР. ${ }^{43}$

ИСПЫТАНИЕ ОПЫТНОГО АТОМНОГО ЗАРЯДА В АВГУСТЕ 1949 Г. НАГЛЯДНО ПОДТВЕРДИЛО ДЕЙСТВЕННОСТЬ СОЗДАННОЙ «УПРАВЛЕНЧЕСКОЙ ВЕРТИКАЛИ». ОНО УКРЕПИЛО УВЕРЕННОСТЬ, ЧТО СПЕЦКОМИТЕТ СПОСОБЕН ОРГАНИЗОВАТЬ РЕШЕНИЕ ЛЮБОЙ ПРОБЛЕМЫ, ИМЕЮЩЕЙ КРИТИЧЕСКОЕ ЗНАЧЕНИЕ ДЛЯ НАРАЩИВАНИЯ ВОЕННО-ОБОРОННОЙ МОЩИ СТРАНЫ. НАИБОЛЕЕ ОСТРО ТОГДА ВОСПРИНИМАЛАСЬ ПОТЕНЦИАЛЬНАЯ ВОЗМОЖНОСТЬ АТОМНОЙ БОМБАРДИРОВКИ СТОЛИЦЫ В СЛУЧАЕ ПРЯМОГО ВОЕННОГО СТОЛКНОВЕНИЯ СОВЕТСКОГО СОЮЗА И СОЕДИНЕННЫХ ШТАТОВ. ДЛЯ ПРЕДОТВРАЩЕНИЯ ТАКОЙ УГРОЗЫ РЕШИЛИ СОЗДАТЬ ЗЕНИТНО-РАКЕТНУЮ СИСТЕМУ ПВО МОСКВЫ (СИСТЕМА «БЕРКУТ»). ЕЕ РАЗРАБОТКУ, ПРОЕКТИРОВАНИЕ И ИЗГОТОВЛЕНИЕ ПОРУЧИЛИ ТРЕТЬЕМУ ГЛАВНОМУ УПРАВЛЕНИЮ, ОРГАНИЗОВАННОМУ В ФЕВРАЛЕ 1951 Г. ПРИ СОВЕТЕ МИНИСТРОВ СССР. РУКОВОДСТВО ВНОВЬ ОБРАЗОВАННОЙ СТРУКТУРОЙ ВОЗЛОЖИЛИ НА СПЕЦКОМИТЕТ, В РЕЗУЛЬТАТЕ ЧЕГО ЕГО КОМПЕТЕНЦИЯ ВЫШЛА ЗА РАМКИ АТОМНОГО ПРОЕКТА. НО В ЯНВАРЕ 1953 Г. ДЕЯТЕЛЬНОСТЬ СПЕЦКОМИТЕТА БЫЛА ПРИОСТАНОВЛЕНА. ДЛЯ НАБЛЮДЕНИЯ ЗА «СПЕЦИАЛЬНЫМИ РАБОТАМИ», ВКЛЮЧАЯ РАЗРАБОТКУ И ПРОИЗВОДСТВО ЯДЕРНОГО ОРУЖИЯ, СИСТЕМЫ ПВО МОСКВЫ, КРЫЛАТЫХ И БАЛЛИСТИЧЕСКИХ РАКЕТ, НАЗНАЧИЛИ ТАК НАЗЫВАЕМУЮ ТРОЙКУ В СОСТАВЕ Л.П. БЕРИИ (ПРЕДСЕДАТЕЛЬ), Г.М. МАЛЕНКОВА, Н.А.БУЛГАНИНА. ПРИЧИНЫ ТАКОГО ШАГА ПОКА НЕИЗВЕСТНЫ. НО КАК БЫ ТАМ НИ БЫЛО, ЧЕРЕЗ ДВА МЕСЯЦА, СРАЗУ ПОСЛЕ СМЕРТИ И.В. СТАЛИНА, СПЕЦКОМИТЕТ ВОССТАНОВИЛИ ПОД ПРЕДСЕДАТЕЛЬСТВОМ ТОГО ЖЕ Л.П. БЕРИИ. ЕМУ ПЕРЕДАЛИ РУКОВОДСТВО « ВСЕМИ СПЕЦИАЛЬНЫМИ РАБОТАМИ ПО АТОМНОЙ ПРОМЫШЛЕННОСТИ, СИСТЕМАМ “БЕРКУТ" И “КОМЕТА” (КРЫЛАТЫМ РАКЕТАМ. - Е.А), РАКЕТАМ ДАЛЬНЕГО ДЕЙСТВИЯ» ${ }^{44}$. ОДНОВРЕМЕННО ОБЪЕДИНИЛИ ПЕРВОЕ И ВТОРОЕ ГЛАВНЫЕ УПРАВЛЕНИЯ.

ТАКАЯ КОНЦЕНТРАЦИЯ ПОЛНОМОЧИЙ В РУКАХ СТРУКТУРЫ, НЕПОДКОНТРОЛЬНОЙ НИКАКИМ ОРГАНАМ ПАРТИЙНО-ГОСУДАРСТВЕННОЙ ВЛАСТИ, ПРОДОЛЖАЛАСЬ НЕДОЛГО. СПЕЦИАЛЬНЫЙ КОМИТЕТ ЛИКВИДИРОВАЛИ 26 ИЮНЯ 1953 Г. РЕШЕНИЕМ ПРЕЗИДИУМА ЦК КПСС, ПРИНЯТЫМ НА ТОМ ЖЕ ЗАСЕДАНИИ, НА КОТОРОМ АРЕСТОВАЛИ Л.П. БЕРИЮ ${ }^{45}$. И ЗДЕСЬ НЕ БЫЛО «НИЧЕГО ЛИЧНОГО». СОЗДАННАЯ СТАЛИНЫМ И БЕРИЕЙ АТОМНАЯ ИМПЕРИЯ СЕРЬЕЗНО УЩЕМЛЯЛА ВЛАСТНЫЕ ПОЛНОМОЧИЯ ВСЕХ РУКОВОДЯЩИХ» СТРУКТУР. УПРАЗДНЕНИЕ СПЕЦКОМИТЕТА ПОЗВОЛИЛО РЕШИТЬ ЭТУ ПРОБЛЕМУ. ДЛЯ РУКОВОДСТВА АТОМНОЙ ОТРАСЛЬЮ СОЗДАЛИ МИНИСТЕРСТВО СРЕДНЕГО МАШИНОСТРОЕНИЯ СССР. В АВГУСТЕ 1953 Г., «УПРАЗДНИЛИ» ИНСТИТУТ УПОЛНОМОЧЕННЫХ СОВЕТА МИНИСТРОВ СССР, ЯВЛЯВШИЙСЯ ДЕЙСТВЕННЫМ ИНСТРУМЕНТОМ СПЕЦКОМИТЕТА В ОСУЩЕСТВЛЕНИИ ЕГО ПОЛИТИКИ. ПРАВО КОНТРОЛЯ ЗА ХОЗЯЙСТВЕННОЙ ДЕЯТЕЛЬНОСТЬЮ В АТОМНОЙ ОТРАСЛИ ПРЕДОСТАВИЛИ ВНОВЬ ОБРАЗОВАННОМУ ПОЛИТУПРАВЛЕНИЮ МИНИСТЕРСТВА СРЕДНЕГО МАШИНОСТРОЕНИЯ СССР, ОБЯЗАВ ЕГО « СВОЕВРЕМЕННО И ПРАВДИВО ДОКЛАДЫВАТЬ В ЦК КПСС И МИНИСТРУ О ПОЛИТИЧЕСКОЙ РАБОТЕ И ПОЛОЖЕНИИ ДЕЛ НА ПРЕДПРИЯТИЯХ, О ЗЛОУПОТРЕБЛЕНИЯХ И НЕДОСТАТКАХ, МОГУЩИХ НАНЕСТИ УЩЕРБ ИНТЕРЕСАМ ГОСУДАРСТВА» ${ }^{46}$. ЭТО РЕШЕНИЕ СТАЛО ВАЖНЫМ ШАГОМ В УСТАНОВЛЕНИИ «ПАРТИЙНОГО КОНТРОЛЯ» ЗА АТОМНОЙ ОТРАСЛЬЮ, КОТОРОГО ТАК ДОБИВАЛСЯ НОВЫЙ ЛИДЕР - Н.С. ХРУЩЕВ. ПЕРЕВОД УПРАВЛЕНИЯ ЯДЕРНО-ОРУЖЕЙНЫМ КОМПЛЕКСОМ НА МИНИСТЕРСКИЙ УРОВЕНЬ ЗАПУСТИЛ ПРОЦЕСС ОБОСОБЛЕНИЯ АТОМНОЙ ОТРАСЛИ. СПУСТЯ ПЯТЬ ЛЕТ НАУЧНЫЙ 
РУКОВОДИТЕЛЬ И ГЛАВНЫЙ КОНСТРУКТОР НИИ-1011 (ВТОРОГО СОВЕТСКОГО ЯДЕРНО-ОРУЖЕЙНОГО ЦЕНТРА) ЧЛ.-КОРР. АН СССР К.И. ЩЕЛКИН ПИСАЛ Н.С. ХРУЩЕВУ О ТОМ, ЧТО ИЗ МИНСРЕДМАША «УШЛО ПОДАВЛЯЮЩЕЕ БОЛЬШИНСТВО ИЗВЕСТНЫХ КРУПНЫХ УЧЕНЫХ...», А «ВСЕ ИНСТИТУТЫ АКАДЕМИИ НАУК, РАНЕЕ ЗАНИМАВШИЕСЯ ПРОБЛЕМАМИ, СВЯЗАННЫМИ С РАЗРАБОТКОЙ ОРУЖИЯ, ТЕПЕРЬ ПРЕКРАТИЛИ ЭТИ РАБОТЫ» ${ }^{47}$. «ОТТОКУ» НАУЧНЫХ СИЛ ИЗ АТОМНОГО ПРОЕКТА СПОСОБСТВОВАЛА ЛИБЕРАЛИЗАЦИЯ ПОЛИТИЧЕСКОГО РЕЖИМА, ДАВАВШАЯ ОПРЕДЕЛЕННУЮ СВОБОДУ ВЫБОРА КАК ОТДЕЛЬНЫМ ЛЮДЯМ, ТАК И ОРГАНИЗАЦИЯМ. ПОХОЖИЕ ПРОЦЕССЫ, ПРАВДА В МЕНЬШИХ МАСШТАБАХ, НАБЛЮДАЛИСЬ И В ПРОИЗВОДСТВЕННОМ СЕКТОРЕ. ЧТОБЫ КОМПЕНСИРОВАТЬ ПОТЕРИ, МИНИСТЕРСТВО СРЕДНЕГО МАШИНОСТРОЕНИЯ ФОРСИРОВАЛО НАРАЩИВАНИЕ ПОТЕНЦИАЛА «СОБСТВЕННЫХ» ПРЕДПРИЯТИЙ И ОРГАНИЗАЦИЙ. ОДНОВРЕМЕННО ПРЕДПРИНИМАЛИСЬ УСИЛИЯ ПО РАСШИРЕНИЮ ИХ СЕТИ. В РЕЗУЛЬТАТЕ ОЧЕНЬ СКОРО МИНСРЕДМАШ ПРЕВРАТИЛСЯ В ОГРОМНОЕ, АВТОРИТЕТНОЕ, НО ПО СВОЕМУ СТАТУСУ ОБЫЧНОЕ МИНИСТЕРСТВО. ЭТО ОЗНАЧАЛО ОТХОД ОТ РАНЕЕ ПРИНЯТОЙ СХЕМЫ ОРГАНИЗАЦИИ РАБОТ, С КОТОРОЙ БЫЛИ СВЯЗАНЫ МНОГИЕ УСПЕХИ АТОМНОГО ПРОЕКТА.

\section{Планирование и организация работы}

ЗА СОДЕРЖАТЕЛЬНУЮ СТОРОНУ АТОМНОГО ПРОЕКТА ОТВЕЧАЛ НАУЧНО-ТЕХНИЧЕСКИЙ СОВЕТ. НА ОСНОВЕ ЕГО РЕКОМЕНДАЦИЙ СПЕЦКОМИТЕТ СТРОИЛ СВОЮ СТРАТЕГИЮ. КОМПЕТЕНЦИЯ СОВЕТА ОХВАТЫВАЛА ШИРОКИЙ КРУГ ВОПРОСОВ - ОТ СОЗДАНИЯ СЫРЬЕВОЙ БАЗЫ И ОСВОЕНИЯ ТЕХНОЛОГИЙ ПОЛУЧЕНИЯ ДЕЛЯЩИХСЯ МАТЕРИАЛОВ (УРАНА-235 И ПЛУТОНИЯ) ДО КОНСТРУИРОВАНИЯ И ИЗГОТОВЛЕНИЯ АТОМНОЙ БОМБЫ. ПРАВДА ВСЕ, ЧТО НЕПОСРЕДСТВЕННО КАСАЛОСЬ ЯДЕРНЫХ БОЕЗАРЯДОВ, СОВЕТ, ПО СООБРАЖЕНИЯМ СЕКРЕТНОСТИ, РАССМАТРИВАЛ НЕ В ПОЛНОМ СОСТАВЕ. ДЛЯ ПОВЫШЕНИЯ ОБОСНОВАННОСТИ СВОИХ ПРЕДЛОЖЕНИЙ ОН ПРИВЛЕКАЛ К ОБСУЖДЕНИЮ ШИРОКИЙ КРУГ УЧЕНЫХ, КОНСТРУКТОРОВ И ПРОИЗВОДСТВЕННИКОВ, РЕГУЛЯРНО ЗАСЛУШИВАЛ ДОКЛАДЫ БЮРО № 2 О ТЕХНИЧЕСКИХ РЕШЕНИЯХ, РЕАЛИЗОВАННЫХ В АМЕРИКАНСКОЙ ЯДЕРНОЙ ПРОГРАММЕ. КОНКРЕТНЫЕ ЗАДАНИЯ ПО АТОМНОМУ ПРОЕКТУ ГОТОВИЛО ПЕРВОЕ ГЛАВНОЕ УПРАВЛЕНИЕ. В СЛУЧАЯХ, КОГДА ЗАТРАГИВАЛИСЬ ИНТЕРЕСЫ МНОГИХ ВЕДОМСТВ, К ЭТОЙ РАБОТЕ ПОДКЛЮЧАЛСЯ СПЕЦИАЛЬНО СОЗДАННЫЙ ОТДЕЛ ГОСПЛАНА. ПОДГОТОВЛЕННЫЕ ПО ТАКОМУ АЛГОРИТМУ ПРОЕКТЫ «РАССМАТРИВАЛИСЬ» СПЕЦКОМИТЕТОМ И ПО ЕГО ПРЕДСТАВЛЕНИЮ УТВЕРЖДАЛИСЬ ПОСТАНОВЛЕНИЯМИ СОВЕТА МИНИСТРОВ СССР. ЗА РЕДКИМ ИСКЛЮЧЕНИЕМ ИХ ПОДПИСЫВАЛ И.В. СТАЛИН, ЧТО ЧАСТО ИНТЕРПРЕТИРУЕТСЯ КАК ЕГО «ПОВСЕДНЕВНОЕ», «ЛИЧНОЕ» РУКОВОДСТВО АТОМНЫМ ПРОЕКТОМ. В РЕАЛЬНОСТИ ЖЕ ОН САНКЦИОНИРОВАЛ ЛИШЬ САМЫЕ ПРИНЦИПИАЛЬНЫЕ РЕШЕНИЯ - О СОЗДАНИИ СПЕЦКОМИТЕТА, ОДНОВРЕМЕННОЙ РАЗРАБОТКЕ АЛЬТЕРНАТИВНЫХ СПОСОБОВ ПРОИЗВОДСТВА ДЕЛЯЩИХСЯ МАТЕРИАЛОВ, МАКСИМАЛЬНОМ ИСПОЛЬЗОВАНИИ АМЕРИКАНСКОГО ОПЫТА КОНСТРУИРОВАНИЯ АТОМНОЙ БОМБЫ, ПРОВЕДЕНИИ ЕЕ ИСПЫТАНИЯ И СЕРИЙНОМ ИЗГОТОВЛЕНИИ « ИЗДЕЛИЙ».

В ОСТАЛЬНЫХ ВОПРОСАХ СПЕЦКОМИТЕТ ДЕЙСТВОВАЛ СОВЕРШЕННО САМОСТОЯТЕЛЬНО. ОФОРМЛЕНИЕ ЕГО ПРЕДЛОЖЕНИЙ В ВИДЕ ПОСТАНОВЛЕНИЙ И РАСПОРЯЖЕНИЙ СОВЕТА МИНИСТРОВ ИМЕЛО ВАЖНОЕ ПРАКТИЧЕСКОЕ ЗНАЧЕНИЕ. ПОРУЧЕНИЯ ЗА ПОДПИСЬЮ И.В. СТАЛИНА СТАНОВИЛИСЬ ДЛЯ ИСПОЛНИТЕЛЕЙ ЗАКОНОМ. САМ ЖЕ ОН, СУДЯ ПО ВСЕМУ, НЕ ВНИКАЛ В СОДЕРЖАНИЕ БОЛЬШИНСТВА ВИЗИРУЕМЫХ РЕШЕНИЙ. ДА ТАКОЕ, 
ДАЖЕ ПРИ ВСЕМ ЖЕЛАНИИ, БЫЛО И НЕВОЗМОЖНО СДЕЛАТЬ: ТОЛЬКО СО ВРЕМЕНИ СОЗДАНИЯ СПЕЦКОМИТЕТА И ДО ИСПЫТАНИЯ ПЕРВОЙ СОВЕТСКОЙ АТОМНОЙ БОМБЫ В АВГУСТЕ 1949 Г. СОВЕТ МИНИСТРОВ СССР ПРИНЯЛ СВЫШЕ ТЫСЯЧИ ПОСТАНОВЛЕНИЙ ПО ВОПРОСАМ РЕАЛИЗАЦИИ АТОМНОЙ ПРОГРАММЫ ${ }^{48}$. ЭТИ ПОСТАНОВЛЕНИЯ И.В. СТАЛИН УТВЕРЖДАЛ «СПИСКОМ», СТАВЯ АВТОГРАФ НА СОПРОВОДИТЕЛЬНЫХ ДОКУМЕНТАХ. ПОДОБНАЯ ПРАКТИКА НЕ ОГРАНИЧИВАЛАСЬ АТОМНЫМ ПРОЕКТОМ. ПО ВОСПОМИНАНИЯМ В.Н. МОЛОТОВА, СОВЕТ МИНИСТРОВ ПРИНИМАЛ ИНОГДА ДО СОТНИ ПОСТАНОВЛЕНИЙ В НЕДЕЛЮ. «ЧИТАТЬ ЕМУ (СТАЛИНУ - Е.Т.) ВСЕ ЭТИ БУМАГИ... БЫЛО БЕССМЫСЛЕННО. ПОТОМУ ЧТО ОН ПРОСТО СТАЛ БЫ БЮРОКРАТОМ», - ГОВОРИЛ В. М. МОЛОТОВ. ПО ЕГО СЛОВАМ, ВСЕ ДЕРЖАЛОСЬ « НА ДОВЕРИИ К... ЗАМАМ, А ТО И НАРКОМАМ, ЧЛЕНАМ ЦК ${ }^{49}$. И НЕСОМНЕННО, В ВОПРОСАХ ОПЕРАТИВНОГО УПРАВЛЕНИЯ АТОМНЫМ ПРОЕКТОМ И.В. СТАЛИН ВСЕЦЕЛО ПОЛАГАЛСЯ НА Л.П. БЕРИЮ. СПЕЦКОМИТЕТ ДЕЙСТВОВАЛ КАК КОЛЛЕГИЯ. НИКАКИЕ УПРАВЛЕНЧЕСКИЕ СТРУКТУРЫ НЕ ИМЕЛИ ПРАВА ВМЕШИВАТЬСЯ В ЕГО ДЕЯТЕЛЬНОСТЬ. ФОНДЫ И ЛИМИТЫ ДЛЯ « СПЕЦИАЛЬНЫХ РАБОТ» УСТАНАВЛИВАЛИСЬ ИМ « ПО ПОТРЕБНОСТИ», НЕЗАВИСИМО ОТ СТЕПЕНИ ОБЕСПЕЧЕНИЯ « ДРУГИХ НУЖД НАРОДНОГО ХОЗЯЙСТВА». МИНИСТЕРСТВАМ И ВЕДОМСТВАМ, СУБЪЕКТАМ ХОЗЯЙСТВОВАНИЯ В ЦЕНТРАЛИЗОВАННОМ ПОРЯДКЕ ВЫДЕЛЯЛИСЬ ДОПОЛНИТЕЛЬНЫЕ ДЕНЕЖНЫЕ СРЕДСТВА, МАТЕРИАЛЫ, ОБОРУДОВАНИЕ И « КОНТИНГЕНТЫ» РАБОЧЕЙ СИЛЫ. В РЯДЕ СЛУЧАЕВ ОНИ ПОКРЫВАЛИ ЗАТРАТЫ ЗА СЧЕТ СОБСТВЕННЫХ ЛИМИТОВ, ЧТО СПЕЦИАЛЬНО ОГОВАРИВАЛОСЬ В СООТВЕТСТВУЮЩИХ ПРАВИТЕЛЬСТВЕННЫХ ПОСТАНОВЛЕНИЯХ. ПОДБОР КОНКРЕТНЫХ ИСПОЛНИТЕЛЕЙ, КАК ПРАВИЛО, ОСУЩЕСТВЛЯЛИ САМИ ВЕДОМСТВА. ЭТО, ОДНАКО, НЕ ИСКЛЮЧАЛО И ИНОГО ВАРИАНТА : ПЕРВОЕ ГЛАВНОЕ УПРАВЛЕНИЕ ИЛИ ЕГО СТРУКТУРЫ НАМЕЧАЛИ НАИБОЛЕЕ « ПОДГОТОВЛЕННЫЕ» ПРЕДПРИЯТИЯ, ЗАТЕМ ДОГОВАРИВАЛИСЬ С ИХ РУКОВОДИТЕЛЯМИ О ПОДКЛЮЧЕНИИ К ВЫПОЛНЕНИЮ КОНКРЕТНЫХ РАБОТ, А МИНИСТЕРСТВАМ И ВЕДОМСТВАМ ОСТАВАЛОСЬ ЛИШЬ ОФОРМИТЬ СВОИМ РЕШЕНИЕМ УЖЕ ДОСТИГНУТОЕ СОГЛАСИЕ.

С САМОГО НАЧАЛА РАБОТЫ ПО АТОМНОМУ ПРОЕКТУ НАПРАВЛЯЛИСЬ ОТДЕЛЬНЫМИ РАСПОРЯЖЕНИЯМИ ПРАВИТЕЛЬСТВА. НО УЖЕ В КОНЦЕ 1945 Г. СПЕЦКОМИТЕТ РЕШИЛ ПЕРЕВЕСТИ ИХ НА «ПЛАНОВЫЕ РЕЛЬСЫ». НАПОМНИМ, ЧТО В СОВЕТСКОЙ МОДЕЛИ УПРАВЛЕНИЯ ЭКОНОМИКОЙ ДЕЙСТВОВАЛА МНОГОУРОВНЕВАЯ СИСТЕМА ПЛАНИРОВАНИЯ. СТРАТЕГИЧЕСКИЕ ЦЕЛИ ЗАДАВАЛИСЬ В ПЯТИЛЕТНИХ ПЛАНАХ - СВОЕОБРАЗНЫХ ОРИЕНТИРАХ НА БУДУЩЕЕ. В НИХ ФИКСИРОВАЛИСЬ ПРИОРИТЕТЫ, ЖЕЛАЕМЫЕ ТЕМПЫ И ПРОПОРЦИИ РАЗВИТИЯ ОТДЕЛЬНЫХ ОТРАСЛЕЙ И НАРОДНОГО ХОЗЯЙСТВА В ЦЕЛОМ, УСТАНАВЛИВАЛИСЬ АГРЕГИРОВАННЫЕ ПОКАЗАТЕЛИ ВЫПУСКА ПРОДУКЦИИ, КАПИТАЛОВЛОЖЕНИЙ И Т.Д. ОПЕРАТИВНОЕ ЖЕ УПРАВЛЕНИЕ ЭКОНОМИКОЙ ОСУЩЕСТВЛЯЛОСЬ ПРИ ПОМОЩИ СРЕДНЕ- И КРАТКОСРОЧНЫХ ПЛАНОВ (ГОДОВЫХ, КВАРТАЛЬНЫХ, МЕСЯЧНЫХ), А ТАКЖЕ СИТУАТИВНО ПРИНИМАЕМЫХ РЕШЕНИЙ ${ }^{50}$. ЗАДАНИЯ УСТАНАВЛИВАЛИ В ХОДЕ СЛОЖНОЙ ПРОЦЕДУРЫ СОГЛАСОВАНИЯ ИНТЕРЕСОВ ВЫШЕ- И НИЖЕСТОЯЩИХ СТРУКТУР, ПРОИЗВОДИТЕЛЕЙ И ПОТРЕБИТЕЛЕЙ, СМЕЖНИКОВ» И Т.Д. ГЛАВНОЕ МЕСТО В ЭТОМ « ТОРГЕ» В СТАЛИНСКУЮ ЭПОХУ ЗАНИМАЛИ ЦЕНТРАЛЬНЫЕ ВЛАСТНЫЕ И УПРАВЛЕНЧЕСКИЕ ОРГАНЫ. ЭТА СХЕМА УПРАВЛЕНИЯ В ОСНОВНОМ СОБЛЮДАЛАСЬ И В АТОМНОМ ПРОЕКТЕ. НО ДЛЯ НЕГО СДЕЛАЛИ ОДНО ВАЖНОЕ ИСКЛЮЧЕНИЕ : «НИКАКИЕ ОРГАНИЗАЦИИ, УЧРЕЖДЕНИЯ И ЛИЦА БЕЗ ОСОБОГО РАЗРЕШЕНИЯ» И.В. СТАЛИНА НЕ ИМЕЛИ « ПРАВА ВМЕШИВАТЬСЯ» В ДЕЛА РУКОВОДСТВА АТОМНОГО ПРОЕКТА. В РЕЗУЛЬТАТЕ ВСЯ ОРГАНИЗАЦИЯ РАБОТЫ СОСРЕДОТОЧИЛАСЬ В СПЕЦИАЛЬНОМ КОМИТЕТЕ. 

ОФИЦИАЛЬНОЕ « РАССМОТРЕНИЕ» В ГОСПЛАНЕ СССР, СРАЗУ ПЕРЕДАЛИ НА УТВЕРЖДЕНИЕ. ДО МИНФИНА ДОВЕЛИ ЛИШЬ ОДНО ТРЕБОВАНИЕ - ПРОФИНАНСИРОВАТЬ ПЛАНИРУЕМЫЕ ЗАТРАТЫ ЗА « СЧЕТ РЕЗЕРВА СОВЕТА МИНИСТРОВ СССР». ПЛАН УТВЕРДИЛИ В ПОСЛЕДНИХ ЧИСЛАХ І КВАРТАЛА РЕШЕНИЕМ СОВМИНА СССР ${ }^{51}$. НО ТАКАЯ ЗАДЕРЖКА НИЧЕГО НЕ МЕНЯЛА : КВАРТАЛЬНОЕ, КАК ВПОСЛЕДСТВИИ И ГОДОВОЕ (НАЧИНАЯ С 1947 Г.), А ЗАТЕМ ПЯТИЛЕТНЕЕ (НА ПЯТУЮ ПЯТИЛЕТКУ), ПЛАНИРОВАНИЕ В АТОМНОМ ПРОЕКТЕ ПОДСТРАИВАЛОСЬ ПОД РЕАЛЬНО СКЛАДЫВАЮЩУЮСЯ СИТУАЦИЮ. С СЕРЕДИНЫ 1946 Г. ВСЕ ПЛАНЫ « СПЕЦРАБОТ» ВКЛЮЧАЛИ ЗАДАНИЯ ДЛЯ СМЕЖНИКОВ ПГУ, ЧТО ПОЗВОЛИЛО УЖЕСТОЧИТЬ КОНТРОЛЬ ЗА ИХ ДЕЯТЕЛЬНОСТЬЮ СО СТОРОНЫ РУКОВОДСТВА АТОМНОГО ПРОЕКТА. ОНИ СОДЕРЖАЛИ СТАНДАРТНЫЙ НАБОР ПОЗИЦИЙ : ПО ВАЛОВОЙ И ТОВАРНОЙ ПРОДУКЦИИ В ЦЕНОВОМ И МАТЕРИАЛЬНОМ ВЫРАЖЕНИИ, ПО СЕБЕСТОИМОСТИ, ПРОИЗВОДИТЕЛЬНОСТИ ТРУДА, ФОНДУ ЗАРПЛАТЫ, ФИНАНСОВОЙ ДЕЯТЕЛЬНОСТИ И КАПИТАЛЬНЫМ РАБОТАМ. С РАЗВЕРТЫВАНИЕМ СЕРИЙНОГО ПРОИЗВОДСТВА « ИЗДЕЛИЙ» СТАЛ ПЛАНИРОВАТЬСЯ ИХ ВЫПУСК. ВСЕ ЭТИ ПОКАЗАТЕЛИ ПОСТОЯННО « КОРРЕКТИРОВАЛИСЬ» СПЕЦКОМИТЕТОМ В СООТВЕТСТВИИ « СО ВНОВЬ ОТКРЫВШИМИСЯ ОБСТОЯТЕЛЬСТВАМИ» И ОЖИДАЕМЫМИ РЕЗУЛЬТАТАМИ. ДЛИТЕЛЬНУЮ ПЕРСПЕКТИВУ. В ХОДЕ ПОДГОТОВКИ ГЕНЕРАЛЬНОГО ПЛАНА РАЗВИТИЯ НАРОДНОГО ХОЗЯЙСТВА МИНИСТЕРСТВАМ И ВЕДОМСТВАМ ПРЕДЛОЖИЛИ ПРЕДСТАВИТЬ СВОИ ПРОЕКТЫ ПЛАНОВ. ОДНАКО ЭТО ПОРУЧЕНИЕ ВЫЗВАЛО ВОЗРАЖЕНИЯ У ПЕРВОГО ГЛАВНОГО УПРАВЛЕНИЯ. Б.Л. ВАННИКОВ И И.В. КУРЧАТОВ ИНФОРМИРОВАЛИ Л.П. БЕРИЮ О ТОМ, ЧТО «В ОТСУТСТВИЕ ДОСТАТОЧНОЙ ЯСНОСТИ ПЕРСПЕКТИВ» ЕГО НЕВОЗМОЖНО ВЫПОЛНИТЬ. САМА ЖЕ « РАЗРАБОТКА ПЛАНА НА 20 ЛЕТ СВЯЗАНА С ОТРЫВОМ АКАДЕМИКА КУРЧАТОВА И ДРУГИХ НУЖНЫХ ЛЮДЕЙ В УЩЕРБ ЗАДАЧАМ, КОТОРЫЕ ПРЕДСТОИТ РЕШИТЬ В БЛИЖАЙШИЕ МЕСЯЦЫ». К ТОМУ ЖЕ, ДОБАВЛЯЛИ ОНИ, «ПО СООБРАЖЕНИЯМ СЕКРЕТНОСТИ» ВКЛЮЧАТЬ СВЕДЕНИЯ О ЯДЕРНО-ОРУЖЕЙНОМ КОМПЛЕКСЕ В ОБЩИЕ ПЛАНЫ НЕЦЕЛЕСООБРАЗНО. Л.П. БЕРИЯ СОГЛАСИЛСЯ С ПОЗИЦИЕЙ ПЕРВОГО ГЛАВНОГО УПРАВЛЕНИЯ. ВСЕ ЗАКОНЧИЛОСЬ ЕГО УСТНЫМ УКАЗАНИЕМ : « 20-ЛЕТНИЙ ПЛАН СПЕЦИАЛЬНЫХ РАБОТ НЕ СОЗДАВАТЬ ${ }^{52}$.

ПРИНЯТЫЙ ПОРЯДОК ПЛАНИРОВАНИЯ И ОРГАНИЗАЦИИ РАБОТЫ СТАВИЛ АТОМНЫЙ ПРОЕКТ В ПРИВИЛЕГИРОВАННОЕ ПОЛОЖЕНИЕ : ДЛЯ ВЫПОЛНЕНИЯ ЕГО ЗАДАНИЙ МОЖНО БЫЛО МОБИЛИЗОВАТЬ ЛЮБЫЕ РЕСУРСЫ. НА ПРАКТИКЕ, ОДНАКО, СУЩЕСТВОВАЛИ ОГРАНИЧЕНИЯ, КОТОРЫЕ НЕ ПОЗВОЛЯЛИ ИГНОРИРОВАТЬ ПОТРЕБНОСТИ (И ИНТЕРЕСЫ) ДРУГИХ ОТРАСЛЕЙ И НАРОДНОГО ХОЗЯЙСТВА В ЦЕЛОМ. В ПЕРВУЮ ОЧЕРЕДЬ ОНИ ЗАДАВАЛИСЬ ПРИНЦИПИАЛЬНЫМИ ОСОБЕННОСТЯМИ УПРАВЛЕНИЯ СОВЕТСКОЙ ЭКОНОМИКОЙ. ВЕДОМСТВА (СНАЧАЛА НАРКОМАТЫ, А ЗАТЕМ МИНИСТЕРСТВА) ИГРАЛИ В НЕЙ КЛЮЧЕВУЮ РОЛЬ : ОНИ ЯВЛЯЛИСЬ ОСНОВНЫМ РЕГУЛЯТОРОМ ЭКОНОМИЧЕСКОГО И НАУЧНО-ТЕХНИЧЕСКОГО РАЗВИТИЯ. НО ОТВЕТСТВЕННОСТЬ ЗА СОСТОЯНИЕ КОНКРЕТНЫХ ПРОИЗВОДСТВ ДИКТОВАЛА ИМ ОПРЕДЕЛЕННУЮ ЛИНИЮ ПОВЕДЕНИЯ. ВО-ПЕРВЫХ, ГЛАВНЫМ ДЛЯ ВЕДОМСТВ БЫЛО РАЗВИТИЕ «СВОИХ» ОТРАСЛЕЙ. ВО-ВТОРЫХ, ОНИ ОБЛАДАЛИ ВЫСОКИМ « ПОРОГОМ ЧУВСТВИТЕЛЬНОСТИ» К ВНЕШНИМ УПРАВЛЕНЧЕСКИМ ВОЗДЕЙСТВИЯМ. И, В-ТРЕТЬИХ, ИХ ПОЗИЦИЯ ВО ВЛАСТНОЙ ИЕРАРХИИ ОПРЕДЕЛЯЛАСЬ МАСШТАБАМИ РЕСУРСОВ, КОТОРЫМИ ОНИ РАСПОРЯЖАЛИСЬ. В РЕЗУЛЬТАТЕ ВЕДОМСТВА, С ОДНОЙ СТОРОНЫ, СТРЕМИЛИСЬ СНИЗИТЬ НАПРЯЖЕННОСТЬ ПЛАНОВ, А С ДРУГОЙ МАКСИМАЛЬНО НАРАСТИТЬ СОБСТВЕННЫЙ ПРОИЗВОДСТВЕННЫЙ ПОТЕНЦИАЛ. ОНИ 
ВЕСЬМА НЕОХОТНО ШЛИ НА ВЫПОЛНЕНИЕ «НЕПРОФИЛЬНЫХ» ЗАДАНИЙ И СОПРОТИВЛЯЛИСЬ ЛЮБЫМ ПОПЫТКАМ ОГРАНИЧИТЬ ИХ ПОЛНОМОЧИЯ В ОТНОШЕНИИ «СВОИХ» ПРЕДПРИЯТИЙ И ОРГАНИЗАЦИЙ. И ТОЛЬКО СИЛЬНЫЙ АДМИНИСТРАТИВНЫЙ НАЖИМ МОГ ЗАСТАВИТЬ ВЕДОМСТВА ДЕЙСТВОВАТЬ ИНАЧЕ.

РУКОВОДСТВО АТОМНОГО ПРОЕКТА БЫЛО ВЫНУЖДЕНО УЧИТЫВАТЬ ЭТИ ОБСТОЯТЕЛЬСТВА. ЕГО ЗАДАНИЯ, « ПОДРАБОТАННЫЕ» ПЕРВЫМ ГЛАВНЫМ УПРАВЛЕНИЕМ, ОБСУЖДАЛИСЬ В СПЕЦКОМИТЕТЕ С ПРИГЛАШЕНИЕМ ВСЕХ РУКОВОДИТЕЛЕЙ ЗАИНТЕРЕСОВАННЫХ ВЕДОМСТВ. ОНИ, КАК ПРАВИЛО, СОГЛАСОВЫВАЛИ ПОДГОТОВЛЕННЫЕ РЕШЕНИЯ. ОДНАКО ИХ АРГУМЕНТАЦИЯ ТАКЖЕ УЧИТЫВАЛАСЬ, ПО КРАЙНЕЙ МЕРЕ, ОНА НЕ ОТВЕРГАЛАСЬ « С ПОРОГА». ОБСУЖДЕНИЯ ПРОХОДИЛИ ОЧЕНЬ ОСТРО. ПО СВИДЕТЕЛЬСТВУ П.А. СУДОПЛАТОВА, РУКОВОДИТЕЛЯ СОВЕТСКОГО АТОМНОГО ШПИОНАЖА», УЧАСТВОВАВШЕГО В ЗАСЕДАНИЯХ СПЕЦКОМИТЕТА, « ЖАРКИЕ СПОРЫ И НЕЛИЦЕПРИЯТНЫЕ ОБЪЯСНЕНИЯ» ЯВЛЯЛИСЬ НОРМОЙ. ТАКИМ ОБРАЗОМ, НАРКОМЫ (МИНИСТРЫ) И ЧЛЕНЫ СПЕЦКОМИТЕТА СТРЕМИЛИСЬ « ВО ЧТО БЫ ТО НИ СТАЛО» ОТСТОЯТЬ СВОИ ПОЗИЦИИ. ДЛЯ СУДОПЛАТОВА, К ПРИМЕРУ, ОКАЗАЛОСЬ « СОВЕРШЕННО НЕОЖИДАННЫМ», ЧТО М.Г. ПЕРВУХИН, ЗАЩИЩАЯ ИНТЕРЕСЫ ХИМИЧЕСКОЙ ПРОМЫШЛЕННОСТИ, РУКОВОДИТЕЛЕМ КОТОРОЙ ОН ЯВЛЯЛСЯ, « ЯРОСТНО НАПАДАЛ» НА ЧЛЕНА ПОЛИТБЮРО ЦК, ПРЕДСЕДАТЕЛЯ ГОСПЛАНА Н.А. ВОЗНЕСЕНСКОГО, ФОРМАЛЬНО «СТАРШЕГО ПО ПОЛОЖЕНИЮ». В ЭТИХ СПОРАХ В КАЧЕСТВЕ АРБИТРА ВЫСТУПАЛ Л.П. БЕРИЯ. БЛАГОДАРЯ СВОЕМУ АВТОРИТЕТУ И ПОЛОЖЕНИЮ ПРЕДСЕДАТЕЛЯ СПЕЦКОМИТЕТА, ОН ДОБИВАЛСЯ «БЕЗУСЛОВНОГО НЕУКОСНИТЕЛЬНОГО ВЫПОЛНЕНИЯ ВСЕХ ДИРЕКТИВ» «ПАРТИИ И ПРАВИТЕЛЬСТВА» ПО СОЗДАНИЮ ЯДЕРНОГО ОРУЖИЯ ${ }^{3}$.

СКАЗАННОЕ ОТНЮДЬ НЕ ОЗНАЧАЕТ, ЧТО СПЕЦКОМИТЕТ СТРЕМИЛСЯ ДОБИТЬСЯ РЕЗУЛЬТАТА ЛЮБОЙ ЦЕНОЙ. ВАЖНЫЙ ОГРАНИЧИТЕЛЬ ЗАПРОСОВ АТОМНОГО ПРОЕКТА НА РЕСУРСЫ БЫЛ ВСТРОЕН В САМУ СТРУКТУРУ ЕГО УПРАВЛЕНИЯ. ВЫСШИЙ РУКОВОДЯЩИЙ ОРГАН АТОМНОГО ПРОЕКТА - СПЕЦКОМИТЕТ - ВКЛЮЧАЛ ЛЮДЕЙ, ОТВЕЧАВШИХ ЗА ОБЩЕЕ СОСТОЯНИЕ ДЕЛ В СТРАНЕ. ТЕ ЖЕ БЕРИЯ, ВОЗНЕСЕНСКИЙ, МАЛЕНКОВ КАК ГОСУДАРСТВЕННЫЕ ДЕЯТЕЛИ ПРОСТО ВЫНУЖДЕНЫ БЫЛИ УЧИТЫВАТЬ НУЖДЫ И ВОЗМОЖНОСТИ ВСЕЙ ЭКОНОМИКИ СТРАНЫ. ОТСЮДА ИХ ВЗВЕШЕННЫЙ ПОДХОД ПРИ ПРИНЯТИИ ЗАТРАТНЫХ РЕШЕНИЙ. ЧАСТО ЭТО ДЕЛАЛОСЬ ВОПРЕКИ ТРЕБОВАНИЯМ ФОРМИРУЮЩЕЙСЯ АТОМНОЙ ОТРАСЛИ. ЯРКИМ СВИДЕТЕЛЬСТВОМ ТОМУ ЯВЛЯЕТСЯ ОБВИНЕНИЕ ЗАМЕСТИТЕЛЯ МИНИСТРА СРЕДНЕГО МАШИНОСТРОЕНИЯ А.П. ЗАВЕНЯГИНА В АДРЕС Л.П. БЕРИИ, ПРОЗВУЧАВШЕЕ НА ИЮЛЬСКОМ (1953 Г.) ПЛЕНУМЕ ЦК КПСС. ОН ИНКРИМИНИРОВАЛ « РАЗОБЛАЧЕННОМУ» ПРЕДСЕДАТЕЛЮ СПЕЦКОМИТЕТА НЕРАЗУМНУЮ ЭКОНОМИЮ СРЕДСТВ. ПОЛЕМИЗИРУЯ С НИМ «ЗАДНИМ ЧИСЛОМ», ЗАВЕНЯГИН УТВЕРЖДАЛ : «ДЕНЬГИ ДЕЙСТВИТЕЛЬНО НУЖНО ЭКОНОМИТЬ, ОНИ НУЖНЫ ДЛЯ РАЗВИТИЯ ПРОМЫШЛЕННОСТИ, ПОДЪЕМА КУЛЬТУРЫ, СЕЛЬСКОГО ХОЗЯЙСТВА. НО В ОБЛАСТИ ИСПОЛЬЗОВАНИЯ АТОМНОЙ ЭНЕРГИИ ЕСТЬ ВОПРОСЫ, В КОТОРЫХ МЫ (РУКОВОДСТВО ПГУ. - Е. А.) НЕ МОГЛИ ПОЗВОЛИТЬ ЧРЕЗМЕРНОЙ ЭКОНОМИИ... КОГДА МЫ СТАВИЛИ ВОПРОС О НОВОМ СТРОИТЕЛЬСТВЕ, БЕРИЯ НАМ ГОВОРИЛ : “К ЧЕРТУ, ВЫ ТРАТИТЕ МНОГО ДЕНЕГ, УКЛАДЫВАЙТЕСЬ В ПЯТИЛЕТКУ”. МЫ НЕ МОГЛИ С ЭТИМ МИРИТЬСЯ, ГОСУДАРСТВО НЕ МОЖЕТ МИРИТЬСЯ. БЕРИЯ ЖЕ ПОВТОРЯЛ НАМ : “К ЧЕРТУ, УКЛАДЫВАЙТЕСЬ В УТВЕРЖДЕННЫЕ ЦИФРЫ"» ${ }^{54}$.

41 ТАК НА ПРАКТИКЕ ПРОИСХОДИЛА ОПТИМИЗАЦИЯ ЗАТРАТ И РЕЗУЛЬТАТОВ В АТОМНОМ ПРОЕКТЕ. ТОЛЬКО ЕГО РУКОВОДСТВО МОГЛО ОГРАНИЧИВАТЬ ТРЕБОВАНИЯ 
ЯДЕРНО-ОРУЖЕЙНОГО КОМПЛЕКСА, НЕ СНИЖАЯ ПЛАНА ВЫПУСКА ГОТОВЫХ « ИЗДЕЛИЙ». ПОХОЖИМ ОБРАЗОМ РЕГУЛИРОВАЛИСЬ ЗАПРОСЫ «СМЕЖНИКОВ» АТОМНОЙ ОТРАСЛИ. ДЛЯ ВЫПОЛНЕНИЯ КОНКРЕТНЫХ ЗАДАНИЙ ИМ ВЫДЕЛЯЛИСЬ ДОПОЛНИТЕЛЬНЫЕ РЕСУРСЫ. ЕСЛИ В ХОДЕ РАБОТЫ ВОЗНИКАЛ ИХ ДЕФИЦИТ, ТО МИНИСТЕРСТВАМ И ВЕДОМСТВАМ ПРЕДЛАГАЛОСЬ РЕШИТЬ ПРОБЛЕМУ ЗА СЧЕТ «ВНУТРЕННИХ РЕЗЕРВОВ». ХОТЯ В ОТДЕЛЬНЫХ СЛУЧАЯХ СПЕЦКОМИТЕТ ШЕЛ НАВСТРЕЧУ ИСПОЛНИТЕЛЯМ И УВЕЛИЧИВАЛ ЛИМИТЫ. ПОДОБНЫЕ ПРАВИЛА ОБЕСПЕЧИВАЛИ ЖЕСТКИЙ КОНТРОЛЬ ЗА ХОДОМ РАБОТ. НО ОНИ НЕ ГАРАНТИРОВАЛИ СБОЕВ В ОСУЩЕСТВЛЕНИИ НАМЕЧЕННЫХ ПЛАНОВ. ОТДЕЛЬНЫЕ ЗАДАНИЯ НЕ ВЫПОЛНЯЛИСЬ В УСТАНОВЛЕННЫЕ СРОКИ. СТРЕМЛЕНИЕ РУКОВОДСТВА АТОМНОГО ПРОЕКТА МИНИМИЗИРОВАТЬ ЗАТРАТЫ ВЕЛО К ПОСТОЯННОЙ НАПРЯЖЕННОСТИ В ИХ РЕСУРСНОМ ОБЕСПЕЧЕНИИ. В ТО ЖЕ ВРЕМЯ РАЗРЕШАЛОСЬ НАЧИНАТЬ СТРОИТЕЛЬСТВО ОСНОВНЫХ «ОБЪЕКТОВ» ЕЩЕ ДО ПОЛНОЙ РАЗРАБОТКИ ПРОЕКТНО-СМЕТНОЙ ДОКУМЕНТАЦИИ. ТАКАЯ ПРАКТИКА СОКРАЩАЛА СРОКИ ИХ ВОЗВЕДЕНИЯ. НО ОНА ОБОРАЧИВАЛАСЬ ДОПОЛНИТЕЛЬНЫМИ ЗАТРАТАМИ. ИНОГДА ДАЖЕ ПРИХОДИЛОСЬ РАЗРУШАТЬ ТО, ЧТО УЖЕ СДЕЛАЛИ, И НАЧИНАТЬ СТРОИТЕЛЬСТВО ЗАНОВО. В ЧАСТНОСТИ, ТАК БЫЛО ПРИ ВОЗВЕДЕНИИ ПЕРВЫХ КОМБИНАТОВ ПО ПРОИЗВОДСТВУ ОБОГАЩЕННОГО УРАНА И НАРАБОТКЕ ПЛУТОНИЯ. ПЕРЕДЕЛКА ПРОЕКТНОЙ ДОКУМЕНТАЦИИ ПРОДОЛЖАЛАСЬ ВПЛОТЬ ДО ИХ СДАЧИ В ЭКСПЛУАТАЦИЮ 5 . БЫЛИ НАРЕКАНИЯ И К КАЧЕСТВУ ПРОДУКЦИИ, ПОСТАВЛЯЕМОЙ СМЕЖНИКАМИ. ИМЕЛИ МЕСТО ФАКТЫ ТАК НАЗЫВАЕМОЙ «БЕСХОЗЯЙСТВЕННОСТИ», НЕЦЕЛЕВОГО ИСПОЛЬЗОВАНИЯ РЕСУРСОВ И Т.П. СВИДЕТЕЛЬСТВА ТОГО ПОВСЕМЕСТНО ВСТРЕЧАЮТСЯ В ПРАВИТЕЛЬСТВЕННЫХ ПОСТАНОВЛЕНИЯХ И РЕШЕНИЯХ СПЕЦКОМИТЕТА. С ОДНОЙ СТОРОНЫ, ЭТО ОБЪЯСНЯЛОСЬ МАСШТАБАМИ И НОВИЗНОЙ ПРОБЛЕМ, УСТАНОВКОЙ НА СКОРЕЙШЕЕ ДОСТИЖЕНИЕ КОНЕЧНОГО РЕЗУЛЬТАТА, С ДРУГОЙ - ЯВЛЯЛОСЬ СЛЕДСТВИЕМ СОВЕТСКОЙ МОДЕЛИ ОРГАНИЗАЦИИ ПРОИЗВОДСТВА : СВОЙСТВЕННЫЕ ЕЙ ДИРЕКТИВНЫЕ МЕТОДЫ РУКОВОДСТВА ПОЗВОЛЯЛИ ОПЕРАТИВНО КОНЦЕНТРИРОВАТЬ РЕСУРСЫ НА НУЖНОМ НАПРАВЛЕНИИ. ОДНАКО ОТСУТСТВИЕ РЫНОЧНЫХ МЕХАНИЗМОВ НЕ ПОЗВОЛЯЛО СКОЛЬКО-НИБУДЬ ТОЧНО ПРОСЧИТАТЬ БАЛАНС ЗАТРАТ И РЕЗУЛЬТАТОВ. В РЕЗУЛЬТАТЕ СПУСКАЕМЫЕ СВЕРХУ УКАЗАНИЯ НЕ ВСЕГДА УЧИТЫВАЛИ РЕАЛЬНЫЕ ПОТРЕБНОСТИ И « ВНУТРЕННИЕ РЕЗЕРВЫ» НЕПОСРЕДСТВЕННЫХ ИСПОЛНИТЕЛЕЙ. ПОСТОЯННО ВОЗНИКАЛИ ТРУДНОСТИ В ПРОЦЕССЕ ОРГАНИЗАЦИИ ВЗАИМОДЕЙСТВИЯ МНОГОЧИСЛЕННЫХ СУБЪЕКТОВ НАУЧНО-ПРОИЗВОДСТВЕННОГО ЦИКЛА. И ВСЕ ЖЕ ДЕЙСТВОВАВШАЯ В АТОМНОМ ПРОЕКТЕ СИСТЕМА МОТИВАЦИИ ТРУДА ОКАЗАЛАСЬ СПОСОБНОЙ СУЩЕСТВЕННО УМЕНЬШИТЬ ОСТРОТУ НАЗВАННЫХ ПРОБЛЕМ. ОНА СМОГЛА ОБЕСПЕЧИТЬ ВЫСОКИЙ УРОВЕНЬ ИСПОЛНИТЕЛЬСКОЙ ДИСЦИПЛИНЫ И ЖЕСТКО СОРИЕНТИРОВАТЬ ВСЕХ СУБЪЕКТОВ НАУЧНО-ТЕХНИЧЕСКОЙ И ПРОИЗВОДСТВЕННОЙ ДЕЯТЕЛЬНОСТИ НА ДОСТИЖЕНИЕ КОНЕЧНОГО РЕЗУЛЬТАТА.

\section{Мотивация труда}

МОТИВАЦИЯ ТРУДА, ИСПОЛЬЗОВАВШАЯСЯ В АТОМНОМ ПРОЕКТЕ, ПРЕСЛЕДОВАЛА СУГУБО ПРАГМАТИЧНЫЕ ЦЕЛИ. ОНА БЫЛА НАПРАВЛЕНА НА МАКСИМИЗАЦИЮ ПРОИЗВОДСТВЕННЫХ УСИЛИЙ. ЭТО РАССМАТРИВАЛОСЬ В КАЧЕСТВЕ НЕОБХОДИМОГО УСЛОВИЯ ДОСТИЖЕНИЯ УСПЕХА. ЧТОБЫ ПОБУДИТЬ ЛЮДЕЙ ПРОЯВЛЯТЬ ИНИЦИАТИВУ, РАБОТАТЬ ИНТЕНСИВНЕЕ И БОЛЬШЕ, ОТВЕТСТВЕННО ОТНОСИТЬСЯ К ПОРУЧЕННОМУ ДЕЛУ ИСПОЛЬЗОВАЛОСЬ СОЧЕТАНИЕ СТАНДАРТНЫХ ДЛЯ СТАЛИНСКОЙ ЭПОХИ МЕТОДОВ : 
МОРАЛЬНОГО ПООЩРЕНИЯ И МАТЕРИАЛЬНОГО СТИМУЛИРОВАНИЯ, ВОСПИТАНИЯ И УБЕЖДЕНИЯ, ПРИНУЖДЕНИЯ И НАСИЛИЯ. ОТЛИЧИЕ ЗАКЛЮЧАЛОСЬ В ПОСЛЕДОВАТЕЛЬНОМ ПРИМЕНЕНИИ ПОЛИТИКИ КНУТА И ПРЯНИКА В ОТНОШЕНИИ ВСЕХ БЕЗ ИСКЛЮЧЕНИЯ РАБОТНИКОВ, А ТАКЖЕ В СНЯТИИ ЛЮБЫХ ОГРАНИЧЕНИЙ КАК ПРИ МАТЕРИАЛЬНОМ ВОЗНАГРАЖДЕНИИ «ОТЛИЧИВШИХСЯ», ТАК И ПРИ НАКАЗАНИИ « НЕРАДИВЫХ». ЕСТЕСТВЕННО, СУЩЕСТВОВАЛА СПЕЦИФИКА В МОТИВАЦИИ ОТДЕЛЬНЫХ КАТЕГОРИЙ ПЕРСОНАЛА. РУКОВОДИТЕЛИ РАЗЛИЧНОГО РАНГА, ВЕДУЩИЕ НАУЧНО-ТЕХНИЧЕСКИЕ СПЕЦИАЛИСТЫ СОСТАВЛЯЛИ ОТНОСИТЕЛЬНО НЕБОЛЬШУЮ ГРУППУ (В ПРЕДЕЛАХ 400-500 ЧЕЛОВЕК), НО ОТ НИХ В РЕШАЮЩЕЙ СТЕПЕНИ ЗАВИСЕЛА СУДЬБА АТОМНОГО ПРОЕКТА. ПОЭТОМУ ЕГО « КЛЮЧЕВЫМ ФИГУРАМ» БЫЛИ СОЗДАНЫ В МАТЕРИАЛЬНОМ ОТНОШЕНИИ ВСЕ УСЛОВИЯ ДЛЯ ИНТЕНСИВНОЙ, ВЫСОКОПРОИЗВОДИТЕЛЬНОЙ РАБОТЫ. ОНИ ИМЕЛИ ВЫСОКИЕ ДОЛЖНОСТНЫЕ ОКЛАДЫ, РЕГУЛЯРНО ПОЛУЧАЛИ СТИМУЛИРУЮЩИЕ ВЫПЛАТЫ. ЗА ОСОБО КРУПНЫЕ РЕЗУЛЬТАТЫ ИМ ВЫДАВАЛИСЬ ОГРОМНЫЕ ПРЕМИИ. В ЧАСТНОСТИ, ПОСЛЕ ПЕРВОГО ИСПЫТАНИЯ АТОМНОЙ БОМБЫ ВЕДУЩИЕ СПЕЦИАЛИСТЫ, РУКОВОДИТЕЛИ «СОПРИЧАСТНЫХ К УСПЕХУ» ПРЕДПРИЯТИЙ И ОРГАНИЗАЦИЙ ПОЛУЧИЛИ ПРЕМИИ, В ДЕСЯТКИ, А ТО И В СОТНИ РАЗ ПРЕВЫШАВШИЕ СРЕДНЕМЕСЯЧНУЮ ЗАРПЛАТУ ВРАЧА ИЛИ РЯДОВОГО ИНЖЕНЕРА ${ }^{56}$. И ИМ БЫЛО НА ЧТО ПОТРАТИТЬ СВОИ ДОХОДЫ : БЛАГОДАРЯ ДОСТУПУ К СПЕЦИАЛЬНЫМ РАСПРЕДЕЛИТЕЛЯМ ПОТРЕБИТЕЛЬСКИХ ТОВАРОВ И ДРУГИМ ПРЕФЕРЕНЦИЯМ, ПРОБЛЕМА « ДЕФИЦИТА» НЕ ИМЕЛА ДЛЯ НИХ ТАКОЙ ОСТРОТЫ, КАК ДЛЯ РЯДОВЫХ ГРАЖДАН.

КОНЕЧНО, МАТЕРИАЛЬНОЕ ПООЩРЕНИЕ ЯВЛЯЛОСЬ НЕ ЕДИНСТВЕННЫМ СТИМУЛОМ. ДЛЯ « РУКОВОДЯЩИХ КАДРОВ» И ВЫСОКОКЛАССНЫХ СПЕЦИАЛИСТОВ УЧАСТИЕ В АТОМНОМ ПРОЕКТЕ ОТКРЫВАЛО ЗАМАНЧИВЫЕ ПЕРСПЕКТИВЫ: ОНИ ПОЛУЧАЛИ ШИРОКИЕ ВОЗМОЖНОСТИ ДЛЯ САМОРЕАЛИЗАЦИИ, ПРОФЕССИОНАЛЬНОГО И КАРЬЕРНОГО РОСТА, УПРОЧЕНИЯ СВОЕГО ВЫСОКОГО СОЦИАЛЬНОГО СТАТУСА. КАК ВСПОМИНАЛ В ОДНОМ ИЗ ПОСЛЕДНИХ ИНТЕРВЬЮ А.Д. САХАРОВ, « ИНТЕРЕС ВЫЗЫВАЛА ГРАНДИОЗНОСТЬ ПРОБЛЕМ, ВОЗМОЖНОСТЬ ПОКАЗАТЬ, НА ЧТО ТЫ СПОСОБЕН, ПРЕЖДЕ ВСЕГО, САМОМУ СЕБЕ ДОКАЗАТЬ ${ }^{57}$. ДЛЯ ИНИЦИАТИВНЫХ И АМБИЦИОЗНЫХ ЛЮДЕЙ ЭТО ЗНАЧИЛО ОЧЕНЬ МНОГО, ПОЗВОЛЯЛО ИМ САМООТВЕРЖЕННО И С ЭНТУЗИАЗМОМ РАБОТАТЬ. ПО СЛОВАМ ОДНОГО ИЗ ГЛАВНЫХ УЧАСТНИКОВ РАЗРАБОТКИ КОНСТРУКЦИИ ПЕРВОЙ СОВЕТСКОЙ АТОМНОЙ БОМБЫ В.А. ЦУКЕРМАНА, « ТРУДИЛИСЬ САМОЗАБВЕННО, С ОГРОМНЫМ УВЛЕЧЕНИЕМ И МОБИЛИЗАЦИЕЙ ВСЕХ ДУШЕВНЫХ И ФИЗИЧЕСКИХ СИЛ» ${ }^{58}$. ТАКОЕ ОТНОШЕНИЕ К РАБОТЕ, СУДЯ ПО СОХРАНИВШИМСЯ ВОСПОМИНАНИЯМ, ЯВЛЯЛОСЬ ПРАВИЛОМ. ОТВЕТСТВЕННОСТИ ДОБАВЛЯЛА УВЕРЕННОСТЬ В ЗНАЧИМОСТИ АТОМНОГО ПРОЕКТА ДЛЯ СУДЕБ СТРАНЫ. ПРЕДСТАВЛЕНИЕ О ТОМ, ЧТО, СОЗДАВАЯ ЯДЕРНОЕ ОРУЖИЕ, ТЫ ТЕМ САМЫМ «ЗАЩИЩАЕШЬ МОСКВУ» ОТ ВОЗМОЖНОЙ УЧАСТИ ХИРОСИМЫ И НАГАСАКИ», БЫЛО ШИРОКО РАСПРОСТРАНЕНО СРЕДИ УЧАСТНИКОВ « АТОМНОЙ ЭПОПЕИ» 59.

44 « ВЫСОКИЕ ЦЕЛИ», СТРЕМЛЕНИЕ К САМОРЕАЛИЗАЦИИ МОТИВИРОВАЛИ ДАЖЕ ТЕХ, КТО КРИТИЧЕСКИ ОТНОСИЛСЯ К СОВЕТСКОЙ ДЕЙСТВИТЕЛЬНОСТИ. СВОЮ РОЛЬ ИГРАЛ И НА УДИВЛЕНИЕ « ЛИБЕРАЛЬНЫЙ» СОЦИАЛЬНО-ПСИХОЛОГИЧЕСКИЙ КЛИМАТ В КОЛЛЕКТИВАХ ОСНОВНЫХ РАЗРАБОТЧИКОВ. ВЕРХОВНАЯ ВЛАСТЬ, ВИДИМО, ПОНИМАЛА, ЧТО ТВОРЧЕСКИЙ ХАРАКТЕР ТРУДА НЕ СОГЛАСУЕТСЯ С ПОКАЗНЫМ ЕДИНОМЫСЛИЕМ И КАЗАРМЕННЫМ ДУХОМ. ПОЭТОМУ МНОГИЕ УЧЕНЫЕ ВОСПРИНИМАЛИ « ЗАКРЫТЫЕ» АТОМНЫЕ ОБЪЕКТЫ, ГДЕ ОНИ БЫЛИ ВЫНУЖДЕНЫ ЖИТЬ И ТРУДИТЬСЯ, КАК ОАЗИСЫ СВОБОДОМЫСЛИЯ. ПО 
КРАЙНЕЙ МЕРЕ, ОБ ЭТОМ ПИШЕТ БОЛЬШИНСТВО МЕМУАРИСТОВ. В ТО ЖЕ ВРЕМЯ НИКТО НЕ ОТРИЦАЕТ, ЧТО РАБОТАТЬ ПРИХОДИЛОСЬ В УСЛОВИЯХ ЖЕСТКОГО АДМИНИСТРАТИВНОГО НАЖИМА. ЗА «СРЫВЫ» ЗАДАНИЙ И «НЕНАДЛЕЖАЩЕЕ» ВЫПОЛНЕНИЕ ПОРУЧЕНИЙ СЛЕДОВАЛИ СЕРЬЕЗНЫЕ САНКЦИИ. СУЩЕСТВУЮТ МНОГОЧИСЛЕННЫЕ СВИДЕТЕЛЬСТВА ТОГО КАК Л.П.БЕРИЯ «РЕЗКО И СТРОГО РАСПРАВЛЯЛСЯ С НЕРАДИВЫМИ ИСПОЛНИТЕЛЯМИ, НЕВЗИРАЯ НА ИХ ЧИНЫ И ПОЛОЖЕНИЕ ${ }^{60}$. ТАК ЖЕ ПОСТУПАЛИ И ДРУГИЕ ВЫСШИЕ РУКОВОДИТЕЛИ АТОМНОГО ПРОЕКТА. ПРАВДА ДЕЛО ПРАКТИЧЕСКИ НИКОГДА НЕ ДОХОДИЛО ДО УГОЛОВНОГО ПРЕСЛЕДОВАНИЯ, НО НИКТО НЕ СОМНЕВАЛСЯ В ТОМ, ЧТО ТАКОЕ ВОЗМОЖНО. ДАЖЕ «КЛЮЧЕВЫЕ ФИГУРЫ» АТОМНОГО ПРОЕКТА НЕ СЧИТАЛИ СЕБЯ ЗАСТРАХОВАННЫМИ ОТ РЕПРЕССИЙ. ВОТ КАК ОБ ЭТОМ ВРЕМЕНИ РАССКАЗЫВАЛ Е.П. СЛАВСКИЙ, СТАВШИЙ ВПОСЛЕДСТВИИ МИНИСТРОМ СРЕДНЕГО МАШИНОСТРОЕНИЯ. БУДУЧИ ЗАМЕСТИТЕЛЕМ НАРКОМА (МИНИСТРА) ЦВЕТНОЙ МЕТАЛЛУРГИИ СССР, ОН ОТВЕЧАЛ ЗА ПРОИЗВОДСТВО ОСОБО ЧИСТОГО ГРАФИТА. ПО ЕГО СЛОВАМ ЭТО БЫЛА «НЕ РАБОТА», А «ХОЖДЕНИЕ ПО ЛЕЗВИЮ БРИТВЫ. МАЛЕЙШИЙ СБОЙ - ЖАКОЙ СБОЙ ПРОИЗОШЕЛ : ПЕРВЫЕ ПАРТИИ ПОЛУЧЕННОГО ГРАФИТА ОКАЗАЛИСЬ СОВЕРШЕННО НЕПРИГОДНЫМИ. ПОД ВОПРОС СТАВИЛАСЬ ВСЯ ПРОГРАММА ПОЛУЧЕНИЯ ПЛУТОНИЯ ДЛЯ АТОМНОЙ БОМБЫ. СЛАВСКОГО ВЫЗВАЛИ В СПЕЦКОМИТЕТ. « МЫ ВЫШЛИ С ЗАСЕДАНИЯ СЛОВНО ЗАНОВО РОДИЛИСЬ, - ВСПОМИНАЛ ЕФИМ ПАВЛОВИЧ. - КАК БУДТО ТОЛЬКО ЧТО СТОЯЛИ НА СТУЛЕ С ПЕТЛЕЙ НА ШЕЕ, И ОСТАВАЛОСЬ ЛИШЬ ЕГО ВЫБИТЬ ИЗ-ПОД НАС. А ТУТ ОКАЗАЛОСЬ, ЧТО СНЯЛИ ПЕТЛЮ, И МЫ ПОШЛИ РАБОТАТЬ. ВОТ КАКОЙ БЫЛ РЕЖИМ ${ }^{61}$. ЕСТЕСТВЕННО, ЧТО ОН УСТРАИВАЛ ДАЛЕКО НЕ ВСЕХ. НО У ПОТЕНЦИАЛЬНЫХ КАНДИДАТОВ НА ОТВЕТСТВЕННЫЕ ДОЛЖНОСТИ В АТОМНОМ ПРОЕКТЕ ОСОБО НЕ СПРАШИВАЛИ СОГЛАСИЯ. ПРЕДЛОЖЕНИЯ, КАК ПРАВИЛО, ПРИНИМАЛИ ВСЕ, ДАЖЕ ЕСЛИ СОМНЕВАЛИСЬ В ЦЕЛЕСООБРАЗНОСТИ ТАКОГО ШАГА. ИНОГДА, ПРАВДА, СИСТЕМА НЕЯВНОГО ПРИНУЖДЕНИЯ НЕ СРАБАТЫВАЛА, И НЕЖЕЛАВШИМ ПО ТЕМ ИЛИ ИНЫМ ПРИЧИНАМ СВЯЗАТЬ СВОЮ СУДЬБУ С АТОМНЫМ ПРОЕКТОМ УДАВАЛОСЬ ОТКАЗАТЬСЯ «ОТ ВЫСОКОГО ДОВЕРИЯ». НО ЭТО, КАК ПРАВИЛО, БЫЛО КОНЦОМ ИХ ПРОФЕССИОНАЛЬНОЙ КАРЬЕРЫ.

РЯДОВЫХ УЧАСТНИКОВ АТОМНОГО ПРОЕКТА - РАБОЧИХ, СЛУЖАЩИХ, ИНЖЕНЕРНО-ТЕХНИЧЕСКИЙ ПЕРСОНАЛ, УПРАВЛЕНЦЕВ НИЗШЕГО И СРЕДНЕГО ЗВЕНА МОТИВИРОВАЛИ ПОДОБНЫМИ ЖЕ СПОСОБАМИ. АДМИНИСТРАТИВНОЕ ПРИНУЖДЕНИЕ И ИДЕОЛОГИЧЕСКАЯ ОБРАБОТКА СОЧЕТАЛИСЬ С ПРОДУМАННОЙ СИСТЕМОЙ МАТЕРИАЛЬНОГО СТИМУЛИРОВАНИЯ И МОРАЛЬНОГО ПООЩРЕНИЯ. НА ВСЕХ ПРЕДПРИЯТИЯХ И В ОРГАНИЗАЦИЯХ, ЗАДЕЙСТВОВАННЫХ В АТОМНОМ ПРОЕКТЕ, КУЛЬТИВИРОВАЛИСЬ ОТНОШЕНИЯ «УДАРНИЧЕСТВА». ТАКАЯ СОЦИАЛЬНО-ПСИХОЛОГИЧЕСКАЯ АТМОСФЕРА ФОРМИРОВАЛА СВОЕОБРАЗНЫЕ ЦЕННОСТНЫЕ ОРИЕНТАЦИИ И МОДЕЛИ ТРУДОВОГО ПОВЕДЕНИЯ. ТАК, НОРМОЙ ЯВЛЯЛОСЬ СТРЕМЛЕНИЕ ДОБИВАТЬСЯ КОНКРЕТНЫХ РЕЗУЛЬТАТОВ. ЭТО ОСОБЕННО ЯРКО ПРОЯВЛЯЛОСЬ НА ОСНОВНЫХ ПРЕДПРИЯТИЯХ ФОРМИРУЮЩЕЙСЯ АТОМНОЙ ОТРАСЛИ. ИХ ТРУДОВЫЕ КОЛЛЕКТИВЫ СОЗДАВАЛИСЬ ПУТЕМ ОТБОРА КВАЛИФИЦИРОВАННЫХ, ДИСЦИПЛИНИРОВАННЫХ, МОРАЛЬНО УСТОЙЧИВЫХ И ПОЛИТИЧЕСКИ БЛАГОНАДЕЖНЫХ КАДРОВ ПО ВСЕЙ СТРАНЕ. ОТДЕЛЬНЫЕ СЛУЧАИ ПРИЕМА НА РАБОТУ БЫВШИХ ЗАКЛЮЧЕННЫХ НЕ ОТМЕНЯЛИ ГЛАВНОГО ПРИНЦИПА : ПРЕДПРИЯТИЯ И ОРГАНИЗАЦИИ АТОМНОЙ ОТРАСЛИ КОМПЛЕКТОВАЛИСЬ ЛЮДЬМИ, НЕ ИМЕВШИМИ СУДИМОСТИ. РАЗЛИЧНЫМ МИНИСТЕРСТВАМ И ВЕДОМСТВАМ, ОБЛАСТНЫМ КОМИТЕТАМ ПАРТИИ СЕКРЕТАРИАТ ЦК ВКП(Б) ПО ПОРУЧЕНИЮ СПЕЦКОМИТЕТА УСТАНАВЛИВАЛ РАЗНАРЯДКУ - 
СКОЛЬКО, КАКИХ СПЕЦИАЛИСТОВ И РАБОЧИХ НУЖНО ОТКОМАНДИРОВАТЬ ДЛЯ НУЖД АТОМНОГО ПРОЕКТА. ПОТЕНЦИАЛЬНЫЕ КАНДИДАТЫ ТЩАТЕЛЬНО ПРОВЕРЯЛИСЬ ОРГАНАМИ ГОСБЕЗОПАСНОСТИ И КАДРОВЫМИ СЛУЖБАМИ ПГУ. ТОЛЬКО ПОСЛЕ ЭТОГО ОНИ НАПРАВЛЯЛИСЬ НА ПРЕДПРИЯТИЯ АТОМНОЙ ОТРАСЛИ. СОГЛАСИЯ САМИХ КАНДИДАТОВ НЕ СПРАШИВАЛИ ${ }^{62}$.

$\begin{array}{llll}\text { ТАКОЙ ПОРЯДОК } & \text { ПРИВЛЕЧЕНИЯ } & \text { КАДРОВ } & \text { МОЖНО }\end{array}$ ДОБРОВОЛЬНО-ПРИНУДИТЕЛЬНЫМ. ПОЭТОМУ ТРУД РАБОТНИКОВ ОСНОВНЫХ ПРОИЗВОДСТВ ВРЯД ЛИ СТОИТ ОТНОСИТЬ К СВОБОДНОМУ В ОБЩЕПРИНЯТОМ СМЫСЛЕ СЛОВА. МОБИЛИЗОВАННЫМ НА ПРЕДПРИЯТИЯ АТОМНОЙ ОТРАСЛИ БЫЛО, КОНЕЧНО, НЕПРОСТО ПРИСПОСОБИТЬСЯ К ЖИЗНИ В «ЗАКРЫТЫХ» ГОРОДАХ, ВОЗВОДИМЫХ ВОЗЛЕ « ОБЪЕКТОВ». СОПРЯЖЕННЫЕ С ЭТИМ НЕУДОБСТВА И НАПРЯЖЕННЫЙ ХАРАКТЕР ТРУДА ВЛАСТИ СТРЕМИЛИСЬ КОМПЕНСИРОВАТЬ ВЫСОКИМ УРОВНЕМ ЗАРПЛАТЫ. ПЕРВЫЕ ЖИТЕЛИ «АТОМНЫХ» ГОРОДОВ СТАЛКИВАЛИСЬ С ОГРОМНЫМИ БЫТОВЫМИ ТРУДНОСТЯМИ: НЕ ХВАТАЛО ДАЖЕ НЕБЛАГОУСТРОЕННОГО ЖИЛЬЯ, ОТСУТСТВОВАЛА СОЦИАЛЬНО-КУЛЬТУРНАЯ ИНФРАСТРУКТУРА. НО, БЛАГОДАРЯ ИНТЕНСИВНОМУ СТРОИТЕЛЬСТВУ, ОСТРОТА ЭТИХ ПРОБЛЕМ БЫСТРО СНИЖАЛАСЬ. ОЧЕНЬ СКОРО «ЗАКРЫТЫЕ» ГОРОДА ЗАМЕТНО ПРЕВЫСИЛИ ОБЩЕСОЮЗНЫЕ СТАНДАРТЫ ПО ОБЕСПЕЧЕННОСТИ ЖИЛЫМ ФОНДОМ И ОБЪЕКТАМИ КУЛЬТУРНО-БЫТОВОГО НАЗНАЧЕНИЯ, А ТАКЖЕ ПО УРОВНЮ БЛАГОУСТРОЙСТВА ${ }^{63}$.

МЕРЫ МАТЕРИАЛЬНОГО И МОРАЛЬНОГО ПООЩРЕНИЯ ПРОИЗВОДСТВЕННОЙ АКТИВНОСТИ ПОДКРЕПЛЯЛИСЬ АДМИНИСТРАТИВНЫМ ПРИНУЖДЕНИЕМ. СИСТЕМАТИЧЕСКОЕ НЕВЫПОЛНЕНИЕ ЗАДАНИЙ ОБОРАЧИВАЛОСЬ ВЫГОВОРАМИ С ПОСЛЕДУЮЩИМ ОГРАНИЧЕНИЕМ ДОСТУПА К РАЗЛИЧНЫМ МАТЕРИАЛЬНЫМ БЛАГАМ, А ИНОГДА И ПЕРЕВОДОМ НА НИЖЕОПЛАЧИВАЕМУЮ РАБОТУ. САМОВОЛЬНОЕ ОСТАВЛЕНИЕ ПРЕДПРИЯТИЯ ОФИЦИАЛЬНО ИМЕНОВАЛОСЬ ДЕЗЕРТИРСТВОМ И ПОДЛЕЖАЛО УГОЛОВНОЙ ОТВЕТСТВЕННОСТИ. В СЛУЧАЕ МНОГОКРАТНЫХ ПРОГУЛОВ ИЛИ ОПОЗДАНИЙ НА РАБОТУ ВИНОВНЫЕ ПРИГОВАРИВАЛИСЬ К ИСПРАВИТЕЛЬНЫМ РАБОТАМ И ДАЖЕ ТЮРЕМНОМУ ЗАКЛЮЧЕНИЮ. ЖЕСТКИЕ САНКЦИИ ЗА НАРУШЕНИЕ ТРУДОВОЙ ДИСЦИПЛИНЫ ХОРОШО ВПИСЫВАЛИСЬ В АТМОСФЕРУ СЕКРЕТНОСТИ, ОКУТЫВАВШУЮ ВСЕ АСПЕКТЫ ПРОИЗВОДСТВЕННОЙ ДЕЯТЕЛЬНОСТИ. ВМЕСТЕ С ИНТЕНСИВНОЙ ПРОПАГАНДОЙ «СОВЕТСКОГО ПАТРИОТИЗМА», С ПОСТОЯННЫМИ НАПОМИНАНИЯМИ О «СЛОЖНОЙ МЕЖДУНАРОДНОЙ ОБСТАНОВКЕ» И Т. П. ЭТО ФОРМИРОВАЛО УСТОЙЧИВОЕ ЧУВСТВО ОТВЕТСТВЕННОСТИ ЗА ПОРУЧЕННОЕ ДЕЛО ПРАКТИЧЕСКИ У ВСЕХ ЗАНЯТЫХ НА ПРЕДПРИЯТИЯХ АТОМНОЙ ИНДУСТРИИ.

СПЕЦИФИЧЕСКУЮ КАТЕГОРИЮ РАБОТНИКОВ АТОМНОЙ ОТРАСЛИ СОСТАВЛЯЛ ТАК НАЗЫВАЕМЫЙ « СПЕЦКОНТИНГЕНТ», Т. Е. ЗАКЛЮЧЕННЫЕ. ВОПРЕКИ РАСПРОСТРАНЕННЫМ ПРЕДСТАВЛЕНИЯМ, ИХ ТРУД НЕ ИСПОЛЬЗОВАЛСЯ НА ОСНОВНОМ ПРОИЗВОДСТВЕ. ИСКЛЮЧЕНИЕМ ЯВЛЯЛИСЬ НАУЧНО-ТЕХНИЧЕСКИЕ ПОДРАЗДЕЛЕНИЯ 9-ГО УПРАВЛЕНИЯ МВД, ВПОСЛЕДСТВИИ ВОШЕДШИЕ В СОСТАВ ПЕРВОГО ГЛАВНОГО УПРАВЛЕНИЯ. ПОЛОВИНУ ИХ ПЕРСОНАЛА СОСТАВЛЯЛИ НЕМЕЦКИЕ ПЛЕННЫЕ И ЗАКЛЮЧЕННЫЕ. В ОСНОВНОМ ЭТО БЫЛИ КВАЛИФИЦИРОВАННЫЕ СПЕЦИАЛИСТЫ. К КОНЦУ 1940-Х ГГ. ИХ ЧИСЛЕННОСТЬ ПРЕВЫСИЛА 200 ЧЕЛОВЕК, ЧТО СОСТАВЛЯЛО ОКОЛО $5 \%$ НАУЧНЫХ И ИНЖЕНЕРНО-ТЕХНИЧЕСКИХ РАБОТНИКОВ, ЗАНЯТЫХ СПЕЦИАЛЬНОЙ» ТЕМАТИКОЙ В НАУЧНО-ИССЛЕДОВАТЕЛЬСКИХ, КОНСТРУКТОРСКИХ И ПРОЕКТНЫХ ОРГАНИЗАЦИЯХ РАЗЛИЧНЫХ МИНИСТЕРСТВ И ВЕДОМСТВ ${ }^{64}$. 
ИНАЯ КАРТИНА НАБЛЮДАЛАСЬ В УРАНОДОБЫВАЮЩЕЙ ПРОМЫШЛЕННОСТИ ВОСТОЧНОЕВРОПЕЙСКИХ СТРАН. ЕЕ ОСНОВНЫЕ МОЩНОСТИ НАХОДИЛИСЬ В ВОСТОЧНОЙ ГЕРМАНИИ. ЗДЕСЬ ЖЕ НАИБОЛЕЕ ШИРОКО ИСПОЛЬЗОВАЛСЯ ПРИНУДИТЕЛЬНЫЙ ТРУД. В КОНЦЕ 1947 Г. ПОЧТИ 80 \% ИЗ 40 ТЫС. РАБОЧИХ АКЦИОНЕРНОГО ОБЩЕСТВА « ВИСМУТ» СОСТАВЛЯЛИ ВОЕННОПЛЕННЫЕ И ДЕПОРТИРОВАННЫЕ ЛИЦА НЕМЕЦКОЙ НАЦИОНАЛЬНОСТИ. НО В НАЧАЛЕ 1950-ХГГ. ИХ ДОЛЯ ЗАМЕТНО УМЕНЬШИЛАСЬ : БОЛЬШИНСТВО ПЕРСОНАЛА, ЧИСЛЕННОСТЬ КОТОРОГО ПРЕВЫСИЛА 150 ТЫС. ЧЕЛОВЕК, УЖЕ СОСТАВЛЯЛИ «ДОБРОВОЛЬЦЫ» ${ }^{65}$. ПОХОЖИМ ОБРАЗОМ РЕШАЛАСЬ КАДРОВАЯ ПРОБЛЕМА В ЧЕХОСЛОВАКИИ. ВНАЧАЛЕ НА УРАНОВЫХ РУДНИКАХ РАБОТАЛИ НЕМЕЦКИЕ ВОЕННОПЛЕННЫЕ. ЗАТЕМ ИХ ЗАМЕНИЛИ ВОЛЬНОНАЕМНЫЕ И ОСУЖДЕННЫЕ ПО ПОЛИТИЧЕСКИМ МОТИВАМ. НА ЗАКЛЮЧЕННЫХ ПРИХОДИЛАСЬ ЗНАЧИТЕЛЬНАЯ ЧАСТЬ « РАБОЧЕЙ СИЛЫ» УРАНОДОБЫВАЮЩИХ ПРЕДПРИЯТИЙ, ЧИСЛЕННОСТЬ КОТОРОЙ В 1953 Г. ПРЕВЫСИЛА 25 ТЫС. ЧЕЛОВЕК ${ }^{66}$.

В СОВЕТСКОМ СОЮЗЕ «СПЕЦКОНТИНГЕНТ» ИСПОЛЬЗОВАЛСЯ НА СТРОИТЕЛЬСТВЕ ОБЪЕКТОВ АТОМНОЙ ИНДУСТРИИ. ИХ В ОСНОВНОМ ВОЗВОДИЛ ГЛАВПРОМСТРОЙ МВД. СВЫШЕ ПОЛОВИНЫ ЕГО ПЕРСОНАЛА СОСТАВЛЯЛИ ЗАКЛЮЧЕННЫЕ, ЧИСЛО КОТОРЫХ В НАЧАЛЕ 1950-ХГГ. ДОСТИГЛО 140 ТЫС. ЧЕЛОВЕК ${ }^{67}$. ДЛЯ ИХ МОТИВАЦИИ ИСПОЛЬЗОВАЛАСЬ СТАНДАРТНАЯ МОДЕЛЬ ГУЛАГА : ЖЕСТКИЙ АДМИНИСТРАТИВНО-РЕПРЕССИВНЫЙ РЕЖИМ ПРИНУЖДЕНИЯ ДОПОЛНЯЛСЯ ПООЩРЕНИЕМ ЗА «УДАРНЫЙ ТРУД». ДЛЯ ВСЕГО «СПЕЦКОНТИНГЕНТА» УСТАНАВЛИВАЛАСЬ $25 \%$ «НАДБАВКА ПРОДОВОЛЬСТВИЯ К ОСНОВНОЙ НОРМЕ ПИТАНИЯ». ДЛЯ «ВЫПОЛНЯВШИХ И ПЕРЕВЫПОЛНЯВШИХ НОРМЫ» ВВОДИЛАСЬ ТАК НАЗЫВАЕМАЯ СИСТЕМА «ЗАЧЕТОВ»: ОНА ПРЕДПОЛАГАЛА « СОКРАЩЕНИЕ СРОКА НАКАЗАНИЯ» В ОПРЕДЕЛЕННОЙ ПРОПОРЦИИ К ОТРАБОТАННОМУ ВРЕМЕНИ ${ }^{68}$. ВООБЩЕ-ТО, ТАКАЯ ПРАКТИКА БЫЛА ЗАПРЕЩЕНА ЗАКОНОДАТЕЛЬНО. СЧИТАЛОСЬ, ЧТО ЕЕ ИСПОЛЬЗОВАНИЕ ВЕДЕТ К ДЕФИЦИТУ ЛАГЕРНОЙ РАБОЧЕЙ СИЛЫ. НО ДЛЯ ЗАКЛЮЧЕННЫХ СИСТЕМА ЗАЧЕТОВ ЯВЛЯЛАСЬ, ПОЖАЛУЙ, ГЛАВНЫМ СТИМУЛОМ К ТРУДУ. ПОЭТОМУ НА ОТДЕЛЬНЫХ « УДАРНЫХ СТРОЙКАХ» ОНА ПРИМЕНЯЛАСЬ ЯВОЧНЫМ ПОРЯДКОМ ${ }^{69}$.

51 К ТАКОВЫМ С САМОГО НАЧАЛА БЫЛИ ОТНЕСЕНЫ « ОБЪЕКТЫ» АТОМНОГО ПРОЕКТА. ДЛЯ « СПЕЦКОНТИНГЕНТА» ЗДЕСЬ СРАЗУ ВВЕЛИ СИСТЕМУ « ЗАЧЕТОВ». НО ЭТО ОБЕРНУЛОСЬ НЕПРЕДВИДЕННЫМИ ПОСЛЕДСТВИЯМИ. ДОСРОЧНО ОСВОБОЖДЕННЫМ ПО СООБРАЖЕНИЯМ СЕКРЕТНОСТИ НЕ РАЗРЕШАЛИ ПОКИДАТЬ « ОБЪЕКТЫ». ИХ ОСТАВЛЯЛИ НА ПРЕЖНЕМ МЕСТЕ РАБОТЫ, ПЕРЕВОДЯ В РАЗРЯД ВОЛЬНОНАЕМНЫХ СТРОИТЕЛЕЙ. ВСКОРЕ КОЛИЧЕСТВО ПОСЛЕДНИХ ПРЕВЫСИЛО 60 ТЫС. ЧЕЛОВЕК ${ }^{70}$. ПО СВОЕМУ СТАТУСУ ЭТА КАТЕГОРИЯ РАБОТНИКОВ, ЗА ИСКЛЮЧЕНИЕМ НЕМНОГИХ СПЕЦИАЛИСТОВ, МАЛО ЧЕМ ОТЛИЧАЛАСЬ ОТ « СПЕЦКОНТИНГЕНТА». ПО БОЛЬШОМУ СЧЕТУ, ИХ ТОЛЬКО НЕ КОНВОИРОВАЛИ ПО ДОРОГЕ НА РАБОТУ. ЕСТЕСТВЕННО, ОЧЕНЬ МНОГИХ ТАКАЯ ЖИЗНЬ НЕ УСТРАИВАЛА. ЭТИМ ОБЪЯСНЯЛСЯ НИЗКИЙ УРОВЕНЬ ПРОИЗВОДСТВЕННОЙ ДИСЦИПЛИНЫ СРЕДИ СТРОИТЕЛЕЙ, РОСТ ПРЕСТУПНОСТИ В «ЗАКРЫТЫХ» ГОРОДАХ И Т.Д. ОБСТАНОВКУ ПЫТАЛИСЬ РАЗРЯДИТЬ АДМИНИСТРАТИВНЫМ СПОСОБОМ. ОСУЖДЕННЫХ ЗА ТЯЖКИЕ ПРЕСТУПЛЕНИЯ ПО ОТБЫТИИ СРОКА НАКАЗАНИЯ СТАЛИ ПЕРЕВОДИТЬ В КАЧЕСТВЕ ВОЛЬНОНАЕМНЫХ В ДАЛЬСТРОЙ МВД СССР. ДРУГИЕ КАТЕГОРИИ ЗАКЛЮЧЕННЫХ, РАБОТАВШИЕ НА ВСПОМОГАТЕЛЬНЫХ ОБЪЕКТАХ, НАЧАЛИ ОСВОБОЖДАТЬ БЕЗ КАКИХ-ЛИБО ОГРАНИЧЕНИЙ 71 . 
НО ПРИНЯТЫЕ МЕРЫ НЕ РЕШАЛИ ГЛАВНОЙ ПРОБЛЕМЫ. « СПЕЦКОНТИНГЕНТ» И ВОЛЬНОНАЕМНЫЕ ИЗ БЫВШИХ ЗАКЛЮЧЕННЫХ КАК РАБОЧАЯ СИЛА» СОВЕРШЕННО НЕ УСТРАИВАЛИ РУКОВОДСТВО АТОМНОГО ПРОЕКТА. В АВГУСТЕ 1948 Г. Б.Л. ВАННИКОВ ПИСАЛ Л.П. БЕРИИ О ТОМ, ЧТО ИХ ИСПОЛЬЗОВАНИЕ, В СИЛУ НИЗКОЙ КВАЛИФИКАЦИИ И НЕГАТИВНОГО ОТНОШЕНИЯ К ТРУДУ, «ЗНАЧИТЕЛЬНО ПОНИЖАЕТ КАЧЕСТВО СТРОИТЕЛЬСТВА И МОНТАЖА, УДОРОЖАЕТ И УСЛОЖНЯЕТ» ВОЗВЕДЕНИЕ « ОБЪЕКТОВ». ОН ПРЕДЛАГАЛ «СМЕНИТЬ КОНТИНГЕНТЫ РАБОЧЕЙ СИЛЫ НА СПЕЦСТРОЙКАХ НА ПРОВЕРЕННЫХ ВОЛЬНОНАЕМНЫХ ИЛИ ВОЕННООБЯЗАННЫХ РАБОЧИХ». ${ }^{72}$ ТАКИЕ ПРОСЬБЫ ВЫСКАЗЫВАЛИСЬ НЕОДНОКРАТНО. ОНИ НАШЛИ ПОДДЕРЖКУ У Л.П. БЕРИИ, КОТОРЫЙ ХОРОШО ПРЕДСТАВЛЯЛ СПЕЦИФИКУ «ЛАГЕРНОЙ ЭКОНОМИКИ». В РЕЗУЛЬТАТЕ БЫЛО ПРИНЯТО РЕШЕНИЕ О ПОСТЕПЕННОЙ ЗАМЕНЕ «СПЕЦКОНТИНГЕНТА» ВОЕННО-СТРОИТЕЛЬНЫМИ ЧАСТЯМИ. В 1952 Г. ИХ ЧИСЛЕННОСТЬ УЖЕ ПРЕВЫСИЛА 50 ТЫС. ЧЕЛОВЕК, А ВО ВТОРОЙ ПОЛОВИНЕ ДЕСЯТИЛЕТИЯ ОНИ СТАЛИ ОСНОВНОЙ РАБОЧЕЙ СИЛОЙ НА СТРОИТЕЛЬСТВЕ ОБЪЕКТОВ АТОМНОЙ ИНДУСТРИИ ${ }^{73}$. ПО СВОЕМУ СТАТУСУ ВОЕННЫЕ СТРОИТЕЛИ ПРИБЛИЖАЛИСЬ К РАБОТНИКАМ ОСНОВНЫХ ПРОИЗВОДСТВ, ДА И МОТИВИРОВАЛИ ИХ СХОДНЫМ ОБРАЗОМ. ТАК ВОЗВОДИМЫЕ ОБЪЕКТЫ АТОМНОЙ ОТРАСЛИ ПОЛУЧИЛИ ДИСЦИПЛИНИРОВАННУЮ РАБОЧУЮ СИЛУ, КОТОРАЯ С ПОНИМАНИЕМ ВОСПРИНИМАЛА ПРИЗЫВЫ К ПРОИЗВОДИТЕЛЬНОМУ И САМООТВЕРЖЕННОМУ ТРУДУ ${ }^{74}$.

ЭФФЕКТИВНОСТЬ ДЕЙСТВОВАВШЕЙ В АТОМНОМ ПРОЕКТЕ СИСТЕМЫ МОТИВАЦИИ ТРУДА, КАК И ОРГАНИЗАЦИИ РАБОТЫ В ЦЕЛОМ, СЛОЖНО ОЦЕНИТЬ ОДНОЗНАЧНО. ОЧЕВИДНО ОДНО : ЦЕЛИ, ЗАДАННЫЕ ПОЛИТИЧЕСКИМ РУКОВОДСТВОМ СТРАНЫ, БЫЛИ ДОСТИГНУТЫ. СОВЕТСКИЙ СОЮЗ ИЗГОТОВИЛ И ИСПЫТАЛ ОПЫТНЫЙ ЯДЕРНЫЙ ЗАРЯД ГОРАЗДО РАНЬШЕ, ЧЕМ ПРЕДПОЛАГАЛИ ЕГО ОППОНЕНТЫ. БОЛЕЕ ТОГО, В СЧИТАННЫЕ ГОДЫ ОН СОЗДАЛ МОЩНЫЙ ЯДЕРНО-ОРУЖЕЙНЫЙ КОМПЛЕКС, СПОСОБНЫЙ СЕРИЙНО ПРОИЗВОДИТЬ САМЫЕ РАЗРУШИТЕЛЬНЫЕ СРЕДСТВА ВООРУЖЕННОЙ БОРЬБЫ. ПРИЧЕМ УЖЕ СЛЕДУЮЩИЕ ЗА ПЕРВОЙ АТОМНОЙ БОМБОЙ «ИЗДЕЛИЯ» ОСНОВЫВАЛИСЬ НЕ СТОЛЬКО НА «ЗАИМСТВОВАННЫХ» У ЗАРУБЕЖНЫХ РАЗРАБОТЧИКОВ ИДЕЯХ, СКОЛЬКО НА ОРИГИНАЛЬНЫХ ПРЕДЛОЖЕНИЯХ СОВЕТСКИХ УЧЕНЫХ И КОНСТРУКТОРОВ. ЭТО СТАЛО ВАЖНЕЙШЕЙ ПРЕДПОСЫЛКОЙ ПРЕВРАЩЕНИЯ СССР В МИРОВУЮ « СВЕРХДЕРЖАВУ». НО ЗДЕСЬ, КОНЕЧНО, СРАЗУ ВОЗНИКАЕТ ВОПРОС О ЦЕНЕ ДОСТИГНУТОГО РЕЗУЛЬТАТА. УЖЕ НА ЗАКАТЕ СОВЕТСКОЙ ЭПОХИ ОДИН ИЗ НАУЧНЫХ РУКОВОДИТЕЛЕЙ АТОМНОГО ПРОЕКТА АКАДЕМИК А.П. АЛЕКСАНДРОВ - УТВЕРЖДАЛ, ЧТО « ЗНАЧИТЕЛЬНАЯ ДОЛЯ ТРУДНОСТЕЙ, ПЕРЕЖИТЫХ НАШИМ НАРОДОМ В ПЕРВЫЕ ПОСЛЕВОЕННЫЕ ГОДЫ», БЫЛА СВЯЗАНА С РАЗВЕРТЫВАНИЕМ « В КРАТЧАЙШИЕ СРОКИ» ПРОИЗВОДСТВА ЯДЕРНОГО ОРУЖИЯ ${ }^{75}$.

СПРАВЕДЛИВОСТЬ ТАКОЙ ОЦЕНКИ ПОДТВЕРЖДАЮТ РАСЧЕТЫ, ВО ЧТО ОБОШЕЛСЯ СТРАНЕ АТОМНЫЙ ПРОЕКТ. ОПУБЛИКОВАНЫ СВЕДЕНИЯ, СОГЛАСНО КОТОРЫМ В ГОДЫ ЧЕТВЕРТОЙ ПЯТИЛЕТКИ ОН ПОГЛОЩАЛ ОКОЛО $3 \%$ КАПИТАЛЬНЫХ ВЛОЖЕНИЙ В НАРОДНОЕ ХОЗЯЙСТВО СТРАНЫ ${ }^{7}$. ДУМАЕТСЯ, ЧТО ЭТИ ДАННЫЕ ЗАНИЖЕНЫ, ПОСКОЛЬКУ НЕ УЧИТЫВАЮТ ЗАТРАТ, ВКЛЮЧЕННЫХ В « ОБЩИЕ ЛИМИТЫ» МИНИСТЕРСТВ-СМЕЖНИКОВ И СРЕДСТВ, ВЛОЖЕННЫХ В РАЗВИТИЕ ОБЕСПЕЧИВАЮЩИХ ПРОИЗВОДСТВ. В ЛЮБОМ СЛУЧАЕ, АТОМНЫЙ ПРОЕКТ ОБЕРНУЛСЯ ОГРОМНОЙ НАГРУЗКОЙ НА ЭКОНОМИКУ И СОЦИАЛЬНУЮ СФЕРУ, ОКАЗАЛ ЗАМЕТНОЕ ВЛИЯНИЕ НА РЕАЛИЗАЦИЮ ПРИНЯТЫХ ПЕРСПЕКТИВНЫХ ПЛАНОВ. В КАЧЕСТВЕ ПРИМЕРА МОЖНО ПРИВЕСТИ ПРОГРАММУ КОМПЛЕКСНОГО 
ХОЗЯЙСТВЕННОГО ОСВОЕНИЯ АНГАРО-ЕНИСЕЙСКОГО РЕГИОНА. В КОНЦЕ 1940-Х ГГ. ЗДЕСЬ НАМЕЧАЛОСЬ ПОСТРОИТЬ КАСКАД КРУПНЫХ ГИДРОЭЛЕКТРОСТАНЦИЙ. ИХ МОЩНОСТИ ПРЕДПОЛАГАЛОСЬ ИСПОЛЬЗОВАТЬ ДЛЯ «ВОВЛЕЧЕНИЯ В ЭКСПЛУАТАЦИЮ ГРОМАДНЫХ» ПРИРОДНЫХ РЕСУРСОВ ВОСТОЧНОЙ СИБИРИ. НО ВСКОРЕ ДЛЯ ВОСПОЛНЕНИЯ ДЕФИЦИТА ОБОГАЩЕННОГО УРАНА РЕШИЛИ ПОСТРОИТЬ АНГАРСКИЙ ЭЛЕКТРОЛИЗНЫЙ ХИМИЧЕСКИЙ КОМБИНАТ - МОЩНЫЙ КОМПЛЕКС ПРОИЗВОДСТВ С ГИГАНТСКИМ ПОТРЕБЛЕНИЕМ ЭЛЕКТРОЭНЕРГИИ. ЭТО, ПО СУТИ, ПЕРЕЧЕРКНУЛО ВСЕ РАНЕЕ ПРИНЯТЫЕ ПЛАНЫ. ЗА КОМБИНАТОМ СРАЗУ ЗАРЕЗЕРВИРОВАЛИ ПОЛОВИНУ МОЩНОСТЕЙ ВОЗВОДИМОЙ ИРКУТСКОЙ ГЭС. ЗАТЕМ ОН СТАЛ ПОТРЕБЛЯТЬ БОЛЬШУЮ ЧАСТЬ ЭЛЕКТРОЭНЕРГИИ, ВЫРАБАТЫВАЕМОЙ БРАТСКОЙ ГЭС. И ТОЛЬКО С ОСВОЕНИЕМ ЦЕНТРИФУЖНОЙ ТЕХНОЛОГИИ ОБОГАЩЕНИЯ УРАНА, ПОЗВОЛЯВШЕЙ СНИЗИТЬ ЭЛЕКТРОПОТРЕБЛЕНИЕ В 20-30 РАЗ, СИТУАЦИЯ КАРДИНАЛЬНО ИЗМЕНИЛАСЬ - МОЖНО БЫЛО ВЕРНУТЬСЯ К РЕАЛИЗАЦИИ РАНЕЕ ПРИНЯТЫХ ПЛАНОВ ${ }^{77}$.

ВМЕСТЕ С ТЕМ НЕ СТОИТ ИГНОРИРОВАТЬ ПОЗИТИВНЫЙ ЭФФЕКТ ОТ РЕАЛИЗАЦИИ АТОМНОГО ПРОЕКТА. НЕСОМНЕННО, ОН ДАЛ МОЩНЫЙ ИМПУЛЬС РАЗВИТИЮ НАУКИ, ТЕХНИКИ, НАУКОЕМКИХ ОТРАСЛЕЙ ПРОМЫШЛЕННОСТИ. В РАМКАХ ПРОЕКТА СУЩЕСТВОВАЛО КАК БЫ ДВА «ВСТРЕЧНЫХ ПОТОКА». С ОДНОЙ СТОРОНЫ, К РАБОТАМ ПРИВЛЕКАЛИСЬ ЛУЧШИЕ НАУЧНО-ТЕХНИЧЕСКИЕ СИЛЫ СТРАНЫ, А С ДРУГОЙНАРАБОТАННЫЕ В НЕМ ИДЕИ И ПОДХОДЫ СТРЕМИЛИСЬ ИСПОЛЬЗОВАТЬ В БОЛЕЕ ШИРОКИХ ЦЕЛЯХ, ЧЕМ СОЗДАНИЕ ЯДЕРНОГО ОРУЖИЯ. ЭТОМУ СПОСОБСТВОВАЛО РУКОВОДСТВО АТОМНЫМ ПРОЕКТОМ В ЛИЦЕ СПЕЦКОМИТЕТА. ОНО ПОЗВОЛЯЛО ОГРАНИЧИТЬ УЗКОВЕДОМСТВЕННЫЕ УСТРЕМЛЕНИЯ, ХОТЯ, КОНЕЧНО, ЗАВЕСА СЕКРЕТНОСТИ СОЗДАВАЛА СЕРЬЕЗНЫЕ ПРЕПЯТСТВИЯ ДЛЯ «ПЕРЕТОКА» ИДЕЙ В ГРАЖДАНСКИЕ ОТРАСЛИ. НАИБОЛЬШЕЕ ВНИМАНИЕ УДЕЛЯЛОСЬ НАРАЩИВАНИЮ НАУЧНО-ТЕХНИЧЕСКОГО И ПРОИЗВОДСТВЕННОГО ПОТЕНЦИАЛА АТОМНОЙ ИНДУСТРИИ. ЕЕ ПРЕДПРИЯТИЯ И ОРГАНИЗАЦИИ ОСНАЩАЛИСЬ НОВЕЙШИМ ОБОРУДОВАНИЕМ, УКОМПЛЕКТОВЫВАЛИСЬ КВАЛИФИЦИРОВАННЫМИ КАДРАМИ. УЖЕ В СЕРЕДИНЕ $1948 Г$. ЗАМЕСТИТЕЛЬ ПРЕДСЕДАТЕЛЯ ГОСПЛАНА СССР Н.А.БОРИСОВ ДОКЛАДЫВАЛ Л.П.БЕРИИ, ЧТО ТАКИЕ ВНОВЬ СОЗДАННЫЕ НАУЧНО-ТЕХНИЧЕСКИЕ ЦЕНТРЫ, КАК ЛАБОРАТОРИЯ № 2 (ИНСТИТУТ АТОМНОЙ ЭНЕРГИИ ИМ. И.В. КУРЧАТОВА), ЛАБОРАТОРИЯ № 3 (ИНСТИТУТ ТЕОРЕТИЧЕСКОЙ И ЭКСПЕРИМЕНТАЛЬНОЙ ФИЗИКИ), КБ-11 (РОССИЙСКИЙ ФЕДЕРАЛЬНЫЙ ЯДЕРНЫЙ ЦЕНТР - ВНИИ ЭКСПЕРИМЕНТАЛЬНОЙ ФИЗИКИ), НИИ-9 (ВНИИ НЕОРГАНИЧЕСКИХ МАТЕРИАЛОВ ИМ. А.А. БОЧВАРА), « МОГУТ УСПЕШНО РЕШАТЬ ЗАДАЧИ КАК ПО ПРОБЛЕМЕ № 1 , ТАК И ПО ДРУГИМ ПРОБЛЕМАМ НАРОДНОГО ХОЗЯЙСТВА» ${ }^{78}$.

В ВЫИГРЫШЕ ОТ УЧАСТИЯ В АТОМНОМ ПРОЕКТЕ ОКАЗАЛСЯ РЯД ИНСТИТУТОВ АКАДЕМИИ НАУК СССР : ФИЗИЧЕСКИЙ ИНСТИТУТ ИМ.С.А. ЛЕБЕДЕВА, ЛЕНИНГРАДСКИЙ ФИЗИКО-ТЕХНИЧЕСКИЙ ИНСТИТУТ, МАТЕМАТИЧЕСКИЙ ИНСТИТУТ ИМ.В.А. СТЕКЛОВА, ИНСТИТУТЫ ХИМИЧЕСКОЙ ФИЗИКИ, ФИЗИЧЕСКОЙ ХИМИИ, ГЕОХИМИИ И АНАЛИТИЧЕСКОЙ ХИМИИ, ГЕОФИЗИКИ И ДР. ОНИ ЗНАЧИТЕЛЬНО, ПОРОЙ В НЕСКОЛЬКО РАЗ, УВЕЛИЧИЛИ ЧИСЛЕННОСТЬ СОТРУДНИКОВ, ПРИБОРНЫЙ ПАРК, РАБОЧИЕ ПЛОЩАДИ. ЭТО ПОСЛУЖИЛО МОЩНЫМ ИМПУЛЬСОМ К РАЗВИТИЮ ОТДЕЛЬНЫХ ИССЛЕДОВАТЕЛЬСКИХ НАПРАВЛЕНИЙ. ПО АВТОРИТЕТНОЙ ОЦЕНКЕ, ОНИ СОХРАНЯЛИ ВЫСОКИЙ УРОВЕНЬ ВПЛОТЬ ДО КОНЦА СОВЕТСКОЙ ЭПОХИ ${ }^{79}$. КРУПНЫЕ ВЛОЖЕНИЯ НАПРАВЛЯЛИСЬ И НА РАЗВИТИЕ ОТРАСЛЕВОЙ НАУКИ. ДЛЯ ПРОЕКТИРОВАНИЯ В ИНТЕРЕСАХ АТОМНОЙ ОТРАСЛИ СПЕЦИАЛЬНОГО ОБОРУДОВАНИЯ, МАШИН, АППАРАТУРЫ И ПРИБОРОВ В МИНИСТЕРСТВАХ МАШИНОСТРОЕНИЯ И ПРИБОРОСТРОЕНИЯ, ТЯЖЕЛОГО МАШИНОСТРОЕНИЯ, 
ПРОМЫШЛЕННОСТИ СРЕДСТВ СВЯЗИ, ХИМИЧЕСКОЙ ПРОМЫШЛЕННОСТИ БЫЛО СОЗДАНО СВЫШЕ 20 КОНСТРУКТОРСКИХ БЮРО И НИИ. ЭТО СПОСОБСТВОВАЛО ПОВЫШЕНИЮ ТЕХНИЧЕСКОГО УРОВНЯ СООТВЕТСТВУЮЩИХ ОТРАСЛЕЙ ПРОМЫШЛЕННОСТИ. НО В ЦЕЛОМ, ПОЛОЖИТЕЛЬНЫЙ ЭФФЕКТ ОТ РЕАЛИЗАЦИИ АТОМНОГО ПРОЕКТА ЛОКАЛИЗОВАЛСЯ В ОГРАНИЧЕННОМ, ПРИОРИТЕТНОМ СЕГМЕНТЕ ПРОИЗВОДСТВА. ОДНАКО ЕГО ОЧЕВИДНЫЕ УСПЕХИ ЕЩЕ НЕ РЕШАЛИ ПРОБЛЕМЫ ОБЕСПЕЧЕНИЯ УСТОЙЧИВОГО ОБЩЕЭКОНОМИЧЕСКОГО РОСТА.

\section{NOTES}

1. * ИССЛЕДОВАНИЕ ОСУЩЕСТВЛЕНО ПРИ ПОДДЕРЖКЕ РОССИЙСКОГО ГОСУДАРСТВЕННОГО НАУЧНОГО ФОНДА (РГНФ), ПРОЕКТ № 14-01-00053.

CM. : D. Holloway, Stalin and the Bomb : the Soviet Union and the Atomic Energy. 1939-1956, New-Haven, 1994 ; В.Н. МИХАЙЛОВ, ГЛ. РЕД., СОЗДАНИЕ ПЕРВОЙ СОВЕТСКОЙ ЯДЕРНОЙ БОМБЫ, М., 1995 ; Е.А. НЕГИН, РУК., Л.П. ГОЛЕУСОВА, Г.Д. КУЛИЧКОВ И ДР., СОВЕТСКИЙ АТОМНЫЙ ПРОЕКТ, НИЖНИЙ НОВГОРОД - АРЗАМАС-16, 1995. Н.С. СИМОНОВ, ВОЕННО-ПРОМЫШЛЕННЫЙ КОМПЛЕКС СССР В 19201950-Е ГОДЫ : ТЕМПЫ ЭКОНОМИЧЕСКОГО РОСТА, СТРУКТУРА, ОРГАНИЗАЦИЯ ПРОИЗВОДСТВА И УПРАВЛЕНИЯ , М., 1996 ; А.К. КРУГЛОВ, ШТАБ АТОМПРОМА, М., 1998 ; Е.Т. АРТЕМОВ, А.Э. БЕДЕЛЬ, УКРОЩЕНИЕ УРАНА, ЕКАТЕРИНБУРГ, 1999 ; И.В. БЫСТРОВА, СОВЕТСКИЙ ВОЕННО-ПРОМЫШЛЕННЫЙ КОМПЛЕКС : ПРОБЛЕМЫ СТАНОВЛЕНИЯ И РАЗВИТИЯ (1930-1980-Е ГОДЫ), М., 2006 ; S.J. Zaloga, The Kremlin's Nuclear Sword: The Rise and Fall of Russia's Strategic Nuclear Forces, Washington D.C., 2002; Н.В. МЕЛЬНИКОВА, ФЕНОМЕН ЗАКРЫТОГО ГОРОДА, ЕКАТЕРИНБУРГ, 2006 ; Н.П. ВОЛОШИН, К ИСТОРИИ ОТЕЧЕСТВЕННОГО АТОМНОГО ПРОЕКТА: КУРС ЛЕКЦИЙ ДЛЯ СЛУШАТЕЛЕЙ УЧЕБНЫХ ЗАВЕДЕНИЙ "РОСАТОМА», М., 2009; ВООРУЖЕНИЕ РОССИИ, В 2-Х ТОМАХ, Т. 1: А.В. МИНАЕВ, РУК. АВТ. КОЛЛЕКТИВА, СОВЕТСКАЯ ВОЕННАЯ МОЩЬ, М., 2010; И.М.САВИЦКИЙ, ВКЛАД ОБОРОННОЙ ПРОМЫШЛЕННОСТИ СИБИРИ В СОЗДАНИЕ РАКЕТНО-ЯДЕРНОГО ЩИТА СССР В ГОДЫ « ХОЛОДНОЙ ВОЙНЫ» (1946-1965 гг.), новосиБиРск, 2011 ; К. Brown, Plutopia: Nuclear Families in Atomic Cities and the Great Soviet and American Plutonium Disasters, New-York, 2013 ; В.Н. НОВОСЕЛОВ, Ю.Ф. НОСАЧ, Б.Н. ЕНТЯКОВ, АТОМНОЕ СЕРДЦЕ РОССИИ, ЧЕЛЯБИНСК, 2014, И ДР.

2. CM. : J.S. Berliner, Factory and Management in the USSR, Cambridge, MA, 1957; M. Lewin, "The Disappearance of Planning in the Plan," Slavic Review, 32, 1973, p. 271-287 ; E. Zaleski, Stalinist Planning for Economic Growth 1933-1952, Chapel Hill, 1980, и дР.

3. Л.Д. РЯБЕВ, ОБЩ. РЕД., АТОМНЫЙ ПРОЕКТ СССР : ДОКУМЕНТЫ И МАТЕРИАЛЫ, В 3 Т., М., 1998-2010.

4. Р.И. ИЛЬКАЕВ, ПРЕД. РЕД. СОВЕТА, В.В. КОНЮК, П.П. МАКСИМЕНКО, А.Д. ПЕЛИПЕНКО, СОСТ., ИСТОРИЯ СОЗДАНИЯ ЯДЕРНОГО ОРУЖИЯ В СССР. 1946-1953 ГОДЫ (В ДОКУМЕНТАХ), В 8 Т., САРОВ (АРЗАМАС-16), 1999-2001.

5. CM.: P. Josephson, Red Atom: Russia's Nuclear Power Program from Stalin to Today, New-York, 1999, p. 13-16.

6. РЯБЕВ, ОБЩ. РЕД., АТОМНЫЙ ПРОЕКТ СССР, Т. 1, Ч. 1, с. 93, 94.

7. НЕГИН, РУК., СОВЕТСКИЙ АТОМНЫЙ ПРОЕКТ, С. 58.

8. СМ. : РЯБЕВ, ОБЩ. РЕД., АТОМНЫЙ ПРОЕКТ СССР, Т. 1, Ч. 1, С. 252 ; Т. 2, КН. 2, с. 415-427.

9. ТАМ ЖЕ. Т. 1, Ч. 1, с. 241-245.

10. ТАМ ЖЕ. С. 260, 267, 268. 
11. ТАМ ЖЕ. С. 265-266.

12. СМ. : КРУГЛОВ, ШТАБ АТОМПРОМА, С. 11.

13. Ф.И. ЧУЕВ, МОЛОТОВ : ПОЛУДЕРЖАВНЫЙ ВЛАСТЕЛИН, М., 2002, с. 108.

14. СМ. : КРУГЛОВ, ШТАБ АТОМПРОМА, С. 79, 80.

15. СМ. : РЯБЕВ, ОБЩ. РЕД., АТОМНЫЙ ПРОЕКТ СССР, Т. 1, Ч. 1, С. 269-271.

16. ТАМ ЖЕ. С. 306-314.

17. ТАМ ЖЕ. С. 268.

18. ТАМ ЖЕ. С. 396, 397.

19. РЯБЕВ, ОБЩ. РЕД., АТОМНЫЙ ПРОЕКТ СССР, Т. 1, Ч. 2. С. 150-153 ; 159-161.

20. ТАМ ЖЕ. С. 289-309.

21. СМ. : РЯБЕВ, ОБЩ. РЕД., АТОМНЫЙ ПРОЕКТ СССР, Т. 2, КН. 1, с. 11-14.

22. Ю.Б. ХАРИТОН, А.А. БРИШ, « ЯДЕРНОЕ ВООРУЖЕНИЕ», В ВООРУЖЕНИЕ РОССИИ, С. 164.

23. СМ. : РЯБЕВ, ОБЩ. РЕД., АТОМНЫЙ ПРОЕКТ СССР, Т. 1, Ч. 2, с. 78, 79 ; К. ЧУПРИН, « УРАН ПО ЛЕНД-ЛИЗУ», СТРАНА РОСАТОМ, 2014 АВГУСТ, № 29 (157).

24. СМ. : ВОЛОШИН, К ИСТОРИИ ОТЕЧЕСТВЕННОГО АТОМНОГО ПРОЕКТА, С. 40-51.

25. РЯБЕВ, ОБЩ. РЕД., АТОМНЫЙ ПРОЕКТ СССР, Т. 2, КН. 1, С. 613-620, 623-629.

26. ТАМ ЖЕ. С. 419.

27. см. : Holloway, Stalin and the Bomb, p. 220, 221 ; В. ПЕЧАТНОВ, « КОНЕЦ АТОМНОЙ МОНОПОЛИИ США», Россия ХХІ, 2004, № 5, с. 66, 67.

28. СМ. : АРТЕМОВ, БЕДЕЛЬ, УКРОЩЕНИЕ УРАНА, С. 75, 120-126.

29. см.: Holloway, Stalin and the Bomb, p.303-319; ВОЛОШИН, К ИСТОРИИ ОТЕЧЕСТВЕННОГО АТОМНОГО ПРОЕКТА, с. 87-115.

30. СМ. : Р.Г. ПИХОЯ, МОСКВА. КРЕМЛЬ. ВЛАСТЬ. СОРОК ЛЕТ ПОСЛЕ ВОЙНЫ, 1945-1985, М., 2007, с. 11 ; О.В. ХЛЕВНЮК, И. ГОРЛИЦКИЙ, ХОЛОДНЫЙ МИР : СТАЛИН И ЗАВЕРШЕНИЕ СТАЛИНСКОЙ ДИКТАТУРЫ, М., 2011, с. 60-74.

31. СМ.: В.П. РАССОХИН, МЕХАНИЗМ ВНЕДРЕНИЯ ДОСТИЖЕНИЙ НАУКИ: ПОЛИТИКА, УПРАВЛЕНИЕ, ПРАВО, М., 1985, С. 55-66 ; В. КОНСТАНТИНОВ, В. НАЙШУЛЬ, ТЕХНОЛОГИЯ ПЛАНОВОГО УПРАВЛЕНИЯ (ПРЕПРИНТ) ЦЭМИ АН СССР, М., 1986, И ДР.

32. РЯБЕВ, ОБЩ. РЕД., АТОМНЫЙ ПРОЕКТ СССР, Т. 2, КН. 4, с. 366-367 ; Т. 2, КН. 5, с. 219-222.

33. РЯБЕВ, ОБЩ. РЕД., АТОМНЫЙ ПРОЕКТ СССР, Т. 2, КН. 5, с. 274-277, 505, 532-534, 558-561.

34. ТАМ ЖЕ. Т. 2, КН. 1, с. 410-4150 ; т. 2, КН. 2, с. 197-201.

35. ТАМ ЖЕ. Т. 2, КН. 2, С. 144-145 ; Т. 2, КН. 3, с. 93-95.

36. СМ. : В.И. ВЕТРОВ, В.В. КРОТКОВ , В.В. КУНИЧЕНКО, « СОЗДАНИЕ ПРЕДПРИЯТИЙ ПО ДОБЫЧЕ И ПЕРЕРАБОТКЕ УРАНОВЫХ РУД», В МИХАЙЛОВ, ГЛ. РЕД., СОЗДАНИЕ ПЕРВОЙ СОВЕТСКОЙ ЯДЕРНОЙ БОМБЫ, С. 191-197.

37. РЯБЕВ, ОБЩ. РЕД., АТОМНЫЙ ПРОЕКТ СССР, Т. 2, КН. 4, С. 388-391.

38. ОЦЕНКА ПО : РЯБЕВ, ОБЩ. РЕД., АТОМНЫЙ ПРОЕКТ СССР, Т. 2, КН. 5, с. 643, 653, 674, 728.

39. СМ. : АРТЕМОВ, БЕДЕЛЬ, УКРОЩЕНИЕ УРАНА, С. 293-295; В.Н. КУЗНЕЦОВ, ЗАКРЫТЫЕ ГОРОДА УРАЛА : ИСТОРИЧЕСКИЕ ОЧЕРКИ, ЕКАТЕРИНБУРГ, 2008, С. 108-115.

40. СМ.: Е.Т. АРТЕМОВ, НАУЧНО-ТЕХНИЧЕСКАЯ ПОЛИТИКА В СОВЕТСКОЙ МОДЕЛИ ПОЗДНЕИНДУСТРИАЛЬНОЙ МОДЕРНИЗАЦИИ, М., 2006, с. 68-70.

41. СМ. : РЯБЕВ, ОБЩ. РЕД., АТОМНЫЙ ПРОЕКТ СССР, Т. 2, КН. 5, с. 750-753, 768, 769, 778, 779, 786, 787.

42. СМ. : А.Н. ЯКОВЛЕВ, ОБЩ. РЕД. АКАД., Д.Г. НАДЖАФОВ, З.С. БЕЛОУСОВА, СОСТ., СТАЛИН И КОСМОПОЛИТИЗМ. 1949-1953 : ДОКУМЕНТЫ АГИТПРОПА ЦК. М., 2005, с. 595-603.

43. СМ. : РЯБЕВ, ОБЩ. РЕД., АТОМНЫЙ ПРОЕКТ СССР, Т. 2, КН. 1, С. 420, 421, 466-470, 500-502.

44. ТАМ ЖЕ. Т. 2, КН. 5, с. 274-277, 505, 532-534.

45. TАM ЖЕ. С. 558-561. 
46. РЯБЕВ, ОБЩ. РЕД., АТОМНЫЙ ПРОЕКТ СССР, С. 579-581.

47. Е.Т. АРТЕМОВ, Н.П. ВОЛОШИН, Б.В. ЛИТВИНОВ, В.И. НИКИТИН, « В ЦЕЛЯХ УСИЛЕНИЯ РАБОТ», УРАЛ. ИСТ. ВЕСТН., 2008, № 3 (20), с. 51.

48. СМ. : Г.А. ГОНЧАРОВ, А.Д. РЯБЕВ, « О СОЗДАНИИ ПЕРВОЙ ОТЕЧЕСТВЕННОЙ АТОМНОЙ БОМБЫ» В РЯБЕВ, ОБЩ. РЕД., АТОМНЫЙ ПРОЕКТ СССР, Т. 2, КН. 6, С. 42.

49. ЧУЕВ, МОЛОТОВ : ПОЛУДЕРЖАВНЫЙ ВЛАСТЕЛИН, С. 315, 316.

50. СМ. : П. ГРЕГОРИ, ПОЛИТИЧЕСКАЯ ЭКОНОМИЯ СТАЛИНИЗМА, М., 2006, С. 159. ОБ ОБЩЕЙ СХЕМЕ ПЛАНИРОВАНИЯ В СТАЛИНСКИЙ ПЕРИОД СМ.: А.М. МАРКЕВИЧ, «БЫЛА ЛИ СОВЕТСКАЯ ЭКОНОМИКА ПЛАНОВОЙ ? ПЛАНИРОВАНИЕ В НАРКОМАТАХ В 1930-Е Г ?.?, Г. », ЭКОНОМИЧЕСКАЯ ИСТОРИЯ. ЕЖЕГОДНИК, 2003, М., 2004, с. 20-54.

51. СМ. : РЯБЕВ, ОБЩ. РЕД., АТОМНЫЙ ПРОЕКТ СССР, Т. 2, КН. 2, С. 156-161.

52. О.В. ХЛЕВНЮК, И. ГОРЛИЦКИЙ, Л.П. КОШЕЛЕВА, СОСТ., ПОЛИТБЮРО, ЦК ВКП(Б) И СОВЕТ МИНИСТРОВ СССР. 1945-1953, М., 2002, С. 243, 244 ; РЯБЕВ, ОБЩ. РЕД., АТОМНЫЙ ПРОЕКТ СССР, Т. 2, Кн. 3, с. 712-719.

53. П.А. СУДОПЛАТОВ, СПЕЦОПЕРАЦИИ. ЛУБЯНКА И КРЕМЛЬ 1930 - 1950 ГОДЫ, М., 2005, с. 329, 330.

54. А.Н. ЯКОВЛЕВ, РЕД., В.НАУМОВ, Ю.СИГАЧЕВ, СОСТ., ЛАВРЕНТИЙ БЕРИЯ. 1953. СТЕНОГРАММА ИЮЛЬСКОГО ПЛЕНУМА ЦК КПСС И ДРУГИЕ ДОКУМЕНТЫ, М., 1999, с. 326, 327.

55. АРТЕМОВ, БЕДЕЛЬ, УКРОЩЕНИЕ УРАНА, С. 29-31; НОВОСЕЛОВ, НОСАЧ, ЕНТЯКОВ, АТОМНОЕ СЕРДЦЕ России, с. 65, 67, 75.

56. ОЦЕНКА ПО : РЯБЕВ, ОБЩ. РЕД., АТОМНЫЙ ПРОЕКТ СССР, Т. 2, КН. 1, С. 53-56 ; Е.Д. ЗУБКОВА, Л.П. КОШЕЛЕВА, Г.А. КУЗНЕЦОВ, СОСТ., СОВЕТСКАЯ ЖИЗНЬ. 1945-1953, М., 2003, С. 501, 502.

57. цит. по : Holloway, Stalin and the Bomb, p. 298.

58. В.А.ЦУКЕРМАН, З.А. АЗАРХ, ЛЮДИ И ВЗРЫВЫ, АРЗАМАС-16, 1994, с. 61.

59. СМ. : Л.В. АЛЬТШУЛЕР, « ВСЯ ЖИЗНЬ В АТОМГРАДЕ», НАУКА И ЖИЗНЬ, 1994, № 2, с. 24-32.

60. А.М. ПЕТРОСЬЯНЦ « РЕШЕНИЕ ЯДЕРНОЙ ПРОБЛЕМЫ В 1943-1946 ГГ.», В МИХАЙЛОВ, ГЛ. РЕД., СОЗДАНИЕ ПЕРВОЙ СОВЕТСКОЙ ЯДЕРНОЙ БОМБЫ, С. 55.

61. ЦИТ. ПО : Б.В. ЛИТВИНОВ, АТОМНАЯ ЭНЕРГИЯ НЕ ТОЛЬКО ДЛЯ ВОЕННЫХ ЦЕЛЕЙ, ЕКАТЕРИНБУРГ, 2002, c. 48.

62. ИЛЬКАЕВ, РЕД., ИСТОРИЯ СОЗДАНИЯ ЯДЕРНОГО ОРУЖИЯ В СССР, Т. 1, КН. 2, САРОВ, 1999, с. 8-37.

63. СМ. : N. Melnikova, «Les artisans du projet nucléaire dans les villes fermées» in Tamara Kondratieva, ed., Les soviétiques, un pouvoir, des régimes, P., 2011, p. 51-75.

64. ОЦЕНКА ПО : РЯБЕВ, ОБЩ. РЕД., АТОМНЫЙ ПРОЕКТ СССР, Т. 2, КН. 4, с. 465-466 ; 725-732.

65. А. ХАЙНЕМАН-ГРУДЕР, « СОВЕТСКИЙ АТОМНЫЙ ПРОЕКТ И НЕХВАТКА УРАНА. ДОБЫЧА УРАНА В ВОСТОЧНОЙ ГЕРМАНИИ И В ЧЕХОСЛОВАКИИ ПОСЛЕ 1945 Г.», В Е.П. ВЕЛИХОВ, РЕД., НАУКА И ОБЩЕСТВО : ИСТОРИЯ СОВЕТСКОГО АТОМНОГО ПРОЕКТА (40-Е-50-Е ГОДЫ) : МЕЖДУНАР. СИМПОЗ. ИСАП-96, М., 1999, С. 337 ; РЯБЕВ, ОБЩ. РЕД., АТОМНЫЙ ПРОЕКТ СССР, Т. 2, КН. 5, С. 728.

66. Ф. ЯНОУХ, «ЧЕХОСЛОВАЦКИЙ УРАН И СОВЕТСКАЯ А-БОМБА», В ВЕЛИХОВ, РЕД., НАУКА И ОБЩЕСТВО, С. 298 ; РЯБЕВ, ОБЩ. РЕД., АТОМНЫЙ ПРОЕКТ СССР, Т. 2, КН. 5, с. 728.

67. РЯБЕВ, ОБЩ. РЕД., АТОМНЫЙ ПРОЕКТ СССР, Т. 2, КН. 5, с. 674.

68. РЯБЕВ, ОБЩ. РЕД., АТОМНЫЙ ПРОЕКТ СССР, Т. 2, КН. 4, с. 198, 199.

69. СМ. : О.В. ХЛЕВНЮК, «ЭКОНОМИКА ОГПУ-НКВД-МВД СССР В 1930 - 1953 ГГ. : МАСШТАБЫ, СТРУКТУРА, ТЕНДЕНЦИИ РАЗВИТИЯ», В Л.И. БОРОДКИН, П. ГРЕГОРИ, О.В. ХЛЕВНЮК, РЕД., ГУЛАГ : ЭКОНОМИКА ПРИНУДИТЕЛЬНОГО ТРУДА, М., 2008, с. 76-77.

70. РЯБЕВ, ОБЩ. РЕД., АТОМНЫЙ ПРОЕКТ СССР, Т. 2, КН. 5, с. 674.

71. ТАМ ЖЕ. Т. 2, КН. 4, С. 521.

72. ТАМ ЖЕ. С. 520, 521, 538, 539. 
73. СМ. : РЯБЕВ, ОБЩ. РЕД., АТОМНЫЙ ПРОЕКТ СССР, Т. 2, КН. 4, с. 156-159 ; И.Е. ДЕРЯБИН, Р.А.ЖУМАНОВ, «СТРОИТЕЛЬНАЯ ИНДУСТРИЯ МИНАТОМА РОССИИ », В А.М.ПЕТРОСЬЯНЦ, ГЛ. РЕД., ЯДЕРНАЯ ИНДУСТРИЯ РОССИИ, М., 2000, с. 815.

74. сМ. : Д.В. РОДЬКИН, « БИТВА ЗА АТОМ. УРАЛЬСКИЙ ФРОНТ», УРАЛ. ИСТ. ВЕСТН., 2013, № 4 (41), c. 73-77.

75. А.П. АЛЕКСАНДРОВ, НАУКА - СТРАНЕ : СТ. И ВЫСТУПЛЕНИЯ, М., 1983.

76. СМ.: СИМОНОВ, ВОЕННО-ПРОМЫШЛЕННЫЙ КОМПЛЕКС СССР В 1920-1950-Е ГОДЫ, С. $240-245$; В.А. ШЕСТАКОВ, СОЦИАЛЬНО-ЭКОНОМИЧЕСКАЯ ПОЛИТИКА СОВЕТСКОГО ГОСУДАРСТВА В 50-Е - СЕРЕДИНЕ 60-Х ГОДов, М., 2006, с. 164.

77. СМ. : Е.Т. АРТЕМОВ, « ВОСТОЧНЫЕ РЕГИОНЫ В ПРОЕКТИРОВКАХ ПРЕДВОЕННОГО ГЕНЕРАЛЬНОГО ХОЗЯЙСТВЕННОГО ПЛАНА», ЭКО. ВСЕРОССИЙСКИЙ ЭКОНОМИЧЕСКИЙ ЖУРНАЛ, 2013, № 1 (463), с. 161-163.

78. РЯБЕВ, ОБЩ. РЕД., АТОМНЫЙ ПРОЕКТ СССР, Т. 2, КН. 3, С. 825.

79. СМ. : А.Е. ВАРШАВСКИЙ, О.С. СИРОТКИН, « НАУЧНО-ТЕХНИЧЕСКИЙ ПОТЕНЦИАЛ», В Д.С. ЛЬВОВ, РУК. АВТ. КОЛЛЕКТИВА, ПУТЬ В ХХІ ВЕК : СТРАТЕГИЧЕСКИЕ ПРОБЛЕМЫ И ПЕРСПЕКТИВЫ РОССИЙСКОЙ экономики, М., 1999, с. 345-347.

\section{RÉSUMÉS}

L'article examine les conditions préalables à l'élaboration et à la mise en œuvre du projet nucléaire soviétique en tant que phénomène de l'économie planifiée. L'étude porte sur la seule année 1953. Elle montre le fonctionnement véritable du système économique socialiste pendant cette période dite classique, et comment cela a permis de réaliser des tâches ambitieuses. Sont analysés les mécanismes de gestion de la prise des décisions, les principes d'organisation et de planification du travail, les méthodes de motivation. Ces analyses permettent de conclure que si la mise en œuvre du projet nucléaire a pesé lourd sur l'économie, elle a cependant donné une impulsion au développement des sciences, des techniques et des industries de haute technologie. Cependant, ces effets positifs n'avaient d'impact que sur une partie limitée de la production et, de fait, ne pouvaient constituer la garantie d'une croissance économique stable.

The paper reviews the preconditions for the development and implementation of the Soviet nuclear project as a phenomenon of centrally planned economy. The study focuses on the year 1953. It shows how the "socialist economic system" in its "classical period" actually operated and identifies the factors enabling it to address ambitious challenges. The author analyses the authorities' decision-making processes, the principles governing the organization and planning of work, and incentives schemes. He concludes that, on the one hand, the nuclear project implementation created a significant additional burden for the economy, but that, on the other hand, it provided a strong impetus for the development of science, technology, and research-intensive industries. However, these positive developments concerned only one segment of production and could not guarantee stable economic growth. 


\section{AUTEUR}

\section{ЕВГЕНИЙ Т. АРТЕМОВ}

ИНСТИТУТ ИСТОРИИ И АРХЕОЛОГИИ УРАЛЬСКОГО ОТДЕЛЕНИЯ РАН, ЕКАТЕРИНБУРГ, iia-history@mail.ru 